\title{
Cosmological constraints from the SDSS luminous red galaxies
}

Max Tegmark, ${ }^{1}$ Daniel J. Eisenstein, ${ }^{2}$ Michael A. Strauss, ${ }^{3}$ David H. Weinberg, ${ }^{4}$ Michael R. Blanton, ${ }^{5}$ Joshua A. Frieman, ${ }^{6,7}$ Masataka Fukugita, ${ }^{8}$ James E. Gunn, ${ }^{3}$ Andrew J. S. Hamilton, ${ }^{9}$ Gillian R. Knapp, ${ }^{3}$ Robert C. Nichol, ${ }^{10}$ Jeremiah P. Ostriker, ${ }^{3}$ Nikhil Padmanabhan, ${ }^{11}$ Will J. Percival, ${ }^{10}$ David J. Schlegel, ${ }^{12}$ Donald P. Schneider, ${ }^{13}$ Roman Scoccimarro, ${ }^{5}$ Uroš Seljak, ${ }^{11,14}$ Hee-Jong Seo, ${ }^{2}$ Molly Swanson, ${ }^{1}$ Alexander S. Szalay, ${ }^{15}$ Michael S. Vogeley, ${ }^{16}$ Jaiyul Yoo, ${ }^{4}$ Idit Zehavi, ${ }^{17}$ Kevork Abazajian, ${ }^{18}$ Scott F. Anderson, ${ }^{19}$ James Annis, ${ }^{7}$ Neta A. Bahcall, ${ }^{3}$ Bruce Bassett, ${ }^{20,21}$ Andreas Berlind, ${ }^{5}$ Jon Brinkmann, ${ }^{22}$ Tamás Budavari, ${ }^{15}$ Francisco Castander, ${ }^{23}$ Andrew Connolly, ${ }^{24}$ Istvan Csabai, ${ }^{15}$ Mamoru Doi, ${ }^{25}$ Douglas P. Finkbeiner, ${ }^{3,26}$ Bruce Gillespie, ${ }^{22}$ Karl Glazebrook, ${ }^{15}$ Gregory S. Hennessy, ${ }^{27}$ David W. Hogg, ${ }^{5}$ Željko Ivezić, ${ }^{3,19}$ Bhuvnesh Jain, ${ }^{28}$ David Johnston, ${ }^{29,30}$ Stephen Kent, ${ }^{7}$ Donald Q. Lamb, ${ }^{6,31}$ Brian C. Lee, ${ }^{12,32}$ Huan Lin, ${ }^{7}$ Jon Loveday, ${ }^{33}$ Robert H. Lupton, ${ }^{3}$ Jeffrey A. Munn, ${ }^{27}$ Kaike Pan, ${ }^{22}$ Changbom Park, ${ }^{34}$ John Peoples, ${ }^{7}$ Jeffrey R. Pier, ${ }^{27}$ Adrian Pope, ${ }^{15}$ Michael Richmond, ${ }^{35}$ Constance Rockosi,${ }^{6}$ Ryan Scranton, ${ }^{24}$ Ravi K. Sheth, ${ }^{28}$ Albert Stebbins, ${ }^{7}$ Christopher Stoughton, ${ }^{7}$ István Szapudi, ${ }^{36}$ Douglas L. Tucker, ${ }^{7}$ Daniel E. Vanden Berk, ${ }^{24}$ Brian Yanny, ${ }^{7}$ and Donald G. York ${ }^{6,31}$

${ }^{1}$ Department of Physics, Massachusetts Institute of Technology, Cambridge, Massachusetts 02139, USA

${ }^{2}$ Department of Astronomy, University of Arizona, Tucson, Arizona 85721, USA

${ }^{3}$ Princeton University Observatory, Princeton, New Jersey 08544, USA

${ }^{4}$ Department of Astronomy, Ohio State University, Columbus, Ohio 43210, USA

${ }^{5}$ Center for Cosmology and Particle Physics, Department of Physics, New York University, 4 Washington Place, New York, New York 10003, USA

${ }^{6}$ Center for Cosmological Physics and Department of Astronomy and Astrophysics, University of Chicago, Chicago, Illinois 60637, USA

${ }^{7}$ Fermi National Accelerator Laboratory, P.O. Box 500, Batavia, Illinois 60510, USA

${ }^{8}$ Institute for Cosmic Ray Research, University of Tokyo, 5-1-5, Kashiwanoha, Kashiwa, Chiba, 277-8582, Japan

${ }^{9}$ JILA and Department of Astrophysical and Planetary Sciences, University of Colorado, Boulder, Colorado 80309, USA

${ }^{10}$ Institute of Cosmology and Gravitation, University of Portsmouth, Portsmouth, P01 2EG, United Kingdom

${ }^{11}$ Department of Physics, Princeton University, Princeton, New Jersey 08544, USA

${ }^{12}$ Lawrence Berkeley National Laboratory, Berkeley, California 94720, USA

${ }^{13}$ Department of Astronomy and Astrophysics, Pennsylvania State University, University Park, Pennsylvania 16802, USA

${ }^{14}$ International Center for Theoretical Physics, Strada Costiera 11, 34014 Trieste, Italy

${ }^{15}$ Department of Physics and Astronomy, The Johns Hopkins University, 3701 San Martin Drive, Baltimore, Maryland 21218, USA

${ }^{16}$ Department of Physics, Drexel University, Philadelphia, Pennsylvania 19104, USA

${ }^{17}$ Department of Astronomy, Case Western Reserve University, 10900 Euclid Avenue, Cleveland, Ohio 44106-7215, USA

${ }^{18}$ Theoretical Division, Los Alamos National Laboratory, Los Alamos, New Mexico 87545, USA

${ }^{19}$ Department of Astronomy, University of Washington, Box 351580, Seattle, Washington 98195, USA

${ }^{20}$ South African Astronomical Observatory, Cape Town, South Africa;

${ }^{21}$ Applied Mathematics Department, University of Cape Town, Cape Town, South Africa

${ }^{22}$ Apache Point Observatory, 2001 Apache Point Road, Sunspot, New Mexico 88349-0059, USA

${ }^{23}$ Institut d'Estudis Espacials de Catalunya/CSIC, Campus UAB, 08034 Barcelona, Spain

${ }^{24}$ University of Pittsburgh, Department of Physics and Astronomy, 3941 O'Hara Street, Pittsburgh, Pennsylvania 15260, USA

${ }^{25}$ Institute of Astronomy, University of Tokyo, Osawa 2-21-1, Mitaka, Tokyo, 181-0015, Japan

${ }^{26}$ Harvard-Smithsonian Center for Astrophysics, 60 Garden Street, MS46, Cambridge, Massachusetts 02138, USA

${ }^{27}$ U.S. Naval Observatory, Flagstaff Station, 10391 W. Naval Observatory Road, Flagstaff, Arizona 86001-8521, USA

${ }^{28}$ Department of Physics, University of Pennsylvania, Philadelphia, Pennsylvania 19104, USA

${ }^{29}$ Jet Propulsion Laboratory, 4800 Oak Grove Drive, Pasadena California, 91109, USA;

${ }^{30}$ California Institute of Technology, 1200 East California Boulevard, Pasadena, California 91125, USA

${ }^{31}$ Enrico Fermi Institute, University of Chicago, Chicago, Illinois 60637, USA

${ }^{32}$ Gatan Inc., Pleasanton, California 94588, USA

${ }^{33}$ Sussex Astronomy Centre, University of Sussex, Falmer, Brighton BN1 9QJ, UK

${ }^{34}$ Department of Astronomy, Seoul National University, 151-742, Korea

${ }^{35}$ Physics Department, Rochester Institute of Technology, 1 Lomb Memorial Dr, Rochester, New York 14623, USA

${ }^{36}$ Institute for Astronomy, University of Hawaii, 2680, Woodlawn Drive, Honolulu, Hawaii 96822, USA (Received 22 August 2006; published 11 December 2006)

We measure the large-scale real-space power spectrum $P(k)$ using luminous red galaxies (LRGs) in the Sloan Digital Sky Survey (SDSS) and use this measurement to sharpen constraints on cosmological parameters from the Wilkinson Microwave Anisotropy Probe (WMAP). We employ a matrix-based power spectrum estimation method using Pseudo-Karhunen-Loève eigenmodes, producing uncorrelated minimum-variance measurements in $20 k$-bands of both the clustering power and its anisotropy due to 
redshift-space distortions, with narrow and well-behaved window functions in the range $0.01 h / \mathrm{Mpc}<$ $k<0.2 h / \mathrm{Mpc}$. Results from the LRG and main galaxy samples are consistent, with the former providing higher signal-to-noise. Our results are robust to omitting angular and radial density fluctuations and are consistent between different parts of the sky. They provide a striking confirmation of the predicted largescale $\Lambda$ CDM power spectrum. Combining only SDSS LRG and WMAP data places robust constraints on many cosmological parameters that complement prior analyses of multiple data sets. The LRGs provide independent cross-checks on $\Omega_{m}$ and the baryon fraction in good agreement with WMAP. Within the context of flat $\Lambda$ CDM models, our LRG measurements complement WMAP by sharpening the constraints on the matter density, the neutrino density and the tensor amplitude by about a factor of 2, giving $\Omega_{m}=$ $0.24 \pm 0.02(1 \sigma), \sum m_{\nu} \lesssim 0.9 \mathrm{eV}(95 \%)$ and $r<0.3(95 \%)$. Baryon oscillations are clearly detected and provide a robust measurement of the comoving distance to the median survey redshift $z=0.35$ independent of curvature and dark energy properties. Within the $\Lambda$ CDM framework, our power spectrum measurement improves the evidence for spatial flatness, sharpening the curvature constraint $\Omega_{\text {tot }}=$ $1.05 \pm 0.05$ from WMAP alone to $\Omega_{\text {tot }}=1.003 \pm 0.010$. Assuming $\Omega_{\text {tot }}=1$, the equation of state parameter is constrained to $w=-0.94 \pm 0.09$, indicating the potential for more ambitious future LRG measurements to provide precision tests of the nature of dark energy. All these constraints are essentially independent of scales $k>0.1 \mathrm{~h} / \mathrm{Mpc}$ and associated nonlinear complications, yet agree well with more aggressive published analyses where nonlinear modeling is crucial.

DOI: 10.1103/PhysRevD.74.123507

PACS numbers: 98.80.Es

\section{INTRODUCTION}

The dramatic recent progress by the Wilkinson Microwave Anisotropy Probe (WMAP) and other experiments [1-4] measuring the cosmic microwave background (CMB) has made non-CMB experiments even more important in the quest to constrain cosmological models and their free parameters. These non-CMB constraints are crucially needed for breaking $\mathrm{CMB}$ degeneracies $[5,6]$; for instance, WMAP alone is consistent with a closed universe with Hubble parameter $h=0.3$ and no cosmological constant [7]. As long as the non-CMB constraints are less reliable and precise than the $\mathrm{CMB}$, they will be the limiting factor and weakest link in the precision cosmology endeavor. Much of the near-term progress in cosmology will therefore be driven by reductions in statistical and systematic uncertainties of non-CMB probes of the cosmic expansion history (e.g., SN Ia) and the matter power spectrum (e.g., Lyman $\alpha$ Forest, galaxy clustering and motions, gravitational lensing, cluster studies and $21 \mathrm{~cm}$ tomography).

The cosmological constraining power of 3-dimensional maps of the Universe provided by galaxy redshift surveys has motivated ever more ambitious observational efforts such as the CfA/UZCPlease supply a definition for CfA/ UZC. [8,9], LCRS [10], PSCz [11], DEEP [12], 2dFGRS [13], and SDSS [14] projects, resulting in progressively more accurate measurements of the galaxy power spectrum $P(k)$ [15-30]. Constraints on cosmological models from these data sets have been most robust when the galaxy clustering could be measured on large scales where one has confidence in the modeling of nonlinear clustering and biasing (e.g., [7,31-42]).

Our goal in this paper therefore is to measure $P(k)$ on large scales using the SDSS galaxy redshift survey in a way that is maximally useful for cosmological parameter esti- mation, and to explore the resulting constraints on cosmological models. The emphasis of our cosmological analysis will be on elucidating the links between cosmological parameters and observable features of the WMAP and SDSS power spectra, and on how these two data sets alone provide tight and robust constraints on many parameters that complement more aggressive but more systematicsprone analyses of multiple data sets.

In a parallel paper, Percival et al. [43] present a power spectrum analysis of the Main Galaxy and LRG samples from the SDSS DR5 data set [44], which is a superset of the data used here. There are a number of differences in the analysis methods. Percival et al. use an FFT-based method to estimate the angle-averaged (monopole) redshift-space galaxy power spectrum. We use a Pseudo-Karhunen-Loève method $[45,46]$ (see further discussion and references below) to estimate the real-space (as opposed to redshiftspace) galaxy power spectrum, using finger-of-god compression and linear theory to remove redshift-space distortion effects. In addition, the many technical decisions that go into these analyses, regarding completeness corrections, angular masks, K-corrections and so forth, were made independently for the two papers, and they present different tests for systematic uncertainties. Despite these many differences of detail, our conclusions agree to the extent that they overlap (as discussed in Sec. III F and Appendix A 1), a reassuring indication of the robustness of the results.

\section{A. Relation between different samples}

The amount of information in a galaxy redshift survey about the galaxy power spectrum $P_{\mathrm{g}}(k)$ and cosmological parameters depends not on the number of galaxies per se, but on the effective volume of the survey, defined by [47] as 


$$
V_{\mathrm{eff}}(k) \equiv \int\left[\frac{\bar{n}(\mathbf{r}) P_{\mathrm{g}}(k)}{1+\bar{n}(\mathbf{r}) P_{\mathrm{g}}(k)}\right]^{2} d^{3} r,
$$

where $\bar{n}(\mathbf{r})$ is the expected number density of galaxies in the survey in the absence of clustering, and the FKP approximation of [19] has been used. The power spectrum error bars scale approximately as $\Delta P_{\mathrm{g}}(k) \propto V_{\text {eff }}(k)^{-1 / 2}$, which for a fixed power $P_{\mathrm{g}}$ is minimized if a fixed total number of galaxies are spaced with density $\bar{n} \sim P_{\mathrm{g}}^{-1}$ [48]. The SDSS Luminous Red Galaxy (LRG) sample was designed $[49,50]$ to contain such "Goldilocks" galaxies with a just-right number density for probing the power around the baryon wiggle scale $k \sim(0.05-0.1) h / \mathrm{Mpc}$. For comparison, the SDSS main galaxy sample [50] is much denser and is dominated by sample variance on these scales, whereas the SDSS quasar sample [51] is much sparser and is dominated by Poisson shot noise. As shown in [36], the effective volume of the LRG sample is about 6 times larger than that of the SDSS main galaxies even though the number of LRGs is an order of magnitude lower, and the LRG volume is over 10 times larger than that of the 2dFGRS. These scalings are confirmed by our results below, which show that $\left(\Delta P_{\mathrm{g}} / P_{\mathrm{g}}\right)^{2}$ on large scales is about 6 times smaller for the SDSS LRGs than for the main-sample galaxies. This gain results both from sampling a larger volume, and from the fact that the LRG are more strongly clustered (biased) than are ordinary galaxies; $P_{\mathrm{g}}$ for LRGs is about 3 times larger than for the main galaxy sample.

We will therefore focus our analysis on the SDSS LRG sample. Although we also measure the SDSS main-sample power spectrum, it adds very little in terms of statistical constraining power; increasing the effective volume by $15 \%$ cuts the error bar $\Delta P$ by only about $(1+0.15)^{1 / 2}-$ $1 \sim 7 \%$. This tiny improvement is easily outweighed by the gain in simplicity from analyzing LRGs alone, where (as we will see) complications such as redshift-dependence of clustering properties are substantially smaller.

A complementary approach implemented by $[41,42]$ is to measure the angular clustering of SDSS LRGs with photometric redshifts, compensating for the loss of radial information with an order of magnitude more galaxies extending out to higher redshift. We will see that this gives comparable or slightly smaller error bars on very large scales $k \leqq 0.02$, but slightly larger error bars on the smaller scales that dominate our cosmological constraints; this is because the number of modes down to a given scale $k$ grows as $k^{3}$ for our three-dimensional spectroscopic analysis, whereas they grow only as $k^{2}$ for a 2-dimensional angular analysis.

\section{B. Relation between different methods}

In the recent literature, two-point galaxy clustering has been quantified using a variety of estimators of both power spectra and correlation functions. The most recent power spectrum measurements for both the 2dFGRS [26,29] and the SDSS $[30,38,43]$ have all interpolated the galaxy density field onto a cubic grid and measured $P(k)$ using a fast fourier transform (FFT).

Appendix A 1 shows that as long as discretization errors from the FFT gridding are negligible, this procedure is mathematically equivalent to measuring the correlation function with a weighted version of the standard "DD - 2DR + RR" method [52,53], multiplying by "RR" and then Fourier transforming. Thus the only advantage of the FFT approach is numerical speedup, and comparing the results with recent correlation function analyses such as [36,54-56] will provide useful consistency checks.

Another approach, pioneered by [45], has been to construct "lossless" estimators of the power spectrum with the smallest error bars that are possible based on information theory $[23,24,27,28,34,45,46,57,58]$. We will travel this complementary route in the present paper, following the matrix-based Pseudo Karhunen-Loève (PKL) eigenmode method described in [28], as it has the following advantages:

(1) It produces power spectrum measurements with uncorrelated error bars.

(2) It produces narrow and well-behaved window functions.

(3) It is lossless in the information theory sense.

(4) It treats redshift distortions without the small-angle approximation.

(5) It readily incorporates the so-called integral constraint [16,59], which can otherwise artificially suppress large-scale power.

(6) It allows testing for systematics that produce excess power in angular or radial modes.

These properties make the results of the PKL-method very easy to interpret and use. The main disadvantage is that the PKL-method is numerically painful to implement and execute; our PKL analysis described below required about a terabyte of disk space for matrix storage and about a year of CPU time, which contributed to the long gestation period of this paper.

The rest of this paper is organized as follows. We describe our galaxy samples and our modeling of them in Sec. II and measure their power spectra in Sec. III. We explore what this does and does not reveal about cosmological parameters in Sec. IV. We summarize our conclusions and place them in context in Sec. V. Further details about analysis techniques are given in Appendix A.

\section{GALAXY DATA}

The SDSS $[14,60]$ uses a mosaic CCD camera [61] on a dedicated telescope [62] to image the sky in five photometric bandpasses denoted $u, g, r, i$, and $z$ [63]. After astrometric calibration [64], photometric data reduction $[65,66]$ and photometric calibration [67-70], galaxies are 
selected for spectroscopic observations [50]. To a good approximation, the main galaxy sample consists of all galaxies with $r$-band apparent Petrosian magnitude $r<$ 17.77 after correction for reddening as per [71]; there are about 90 such galaxies per square degree, with a median redshift of 0.1 and a tail out to $z \sim 0.25$. Galaxy spectra are also measured for the LRG sample [49], targeting an additional $\sim 12$ galaxies per square degree, enforcing $r<19.5$ and color-magnitude cuts described in [36,49] that select mainly luminous elliptical/early type galaxies at redshifts up to $\sim 0.5$. These targets are assigned to spectroscopic plates of diameter $2.98^{\circ}$ into which 640 optical fibers are plugged by an adaptive tiling algorithm [72], feeding a pair of CCD spectrographs [73], after which the spectroscopic data reduction and redshift determination are performed by automated pipelines. The rms galaxy redshift errors are of order $30 \mathrm{~km} / \mathrm{s}$ for main galaxies and $50 \mathrm{~km} / \mathrm{s}$ for LRGs [49], hence negligible for the purposes of the present paper.

Our analysis is based on 58360 LRGs and 285804 main galaxies (the "safe13" cut) from the 390, 288 galaxies in the 4th SDSS data release ("DR4") [74], processed via the SDSS data repository at New York University [75]. The details of how these samples were processed and modeled are given in Appendix A of [28] and in [36]. The bottom line is that each sample is completely specified by three entities:

(1) The galaxy positions (RA, Dec and comoving redshift-space distance $r$ for each galaxy),

(2) The radial selection function $\bar{n}(r)$, which gives the expected number density of galaxies as a function of distance,

(3) The angular selection function $\bar{n}(\hat{\mathbf{r}})$, which gives the completeness as a function of direction in the sky, specified in a set of spherical polygons [76].

Our samples are constructed so that their threedimensional selection function is separable, i.e., simply the product $\bar{n}(\mathbf{r})=\bar{n}(\hat{\mathbf{r}}) \bar{n}(r)$ of an angular and a radial part; here $r \equiv|\mathbf{r}|$ and $\hat{\mathbf{r}} \equiv \mathbf{r} / r$ are the comoving radial distance and the unit vector corresponding to the position r. The effective sky area covered is $\Omega \equiv \int \bar{n}(\hat{\mathbf{r}}) d \Omega \approx$ 4259 square degrees, and the typical completeness $\bar{n}(\hat{\mathbf{r}})$ exceeds $90 \%$. The radial selection function $\bar{n}(r)$ for the LRGs is the one constructed and described in detail in $[36,56]$, based on integrating an empirical model of the luminosity function and color distribution of the LRGs against the luminosity-color selection boundaries of the sample. Figure 1 shows that it agrees well with the observed galaxy distribution. The conversion from redshift $z$ to comoving distance was made for a flat $\Lambda \mathrm{CDM}$ cosmological model with $\Omega_{m}=0.25$. If a different cosmological model is used for this conversion, then our measured dimensionless power spectrum $k^{3} P(k)$ is dilated very slightly (by $\lesssim 1 \%$ for models consistent with our measurements) along the $k$ axis; we include this dilation effect in our cosmological parameter analysis as described in Appendix A 4.

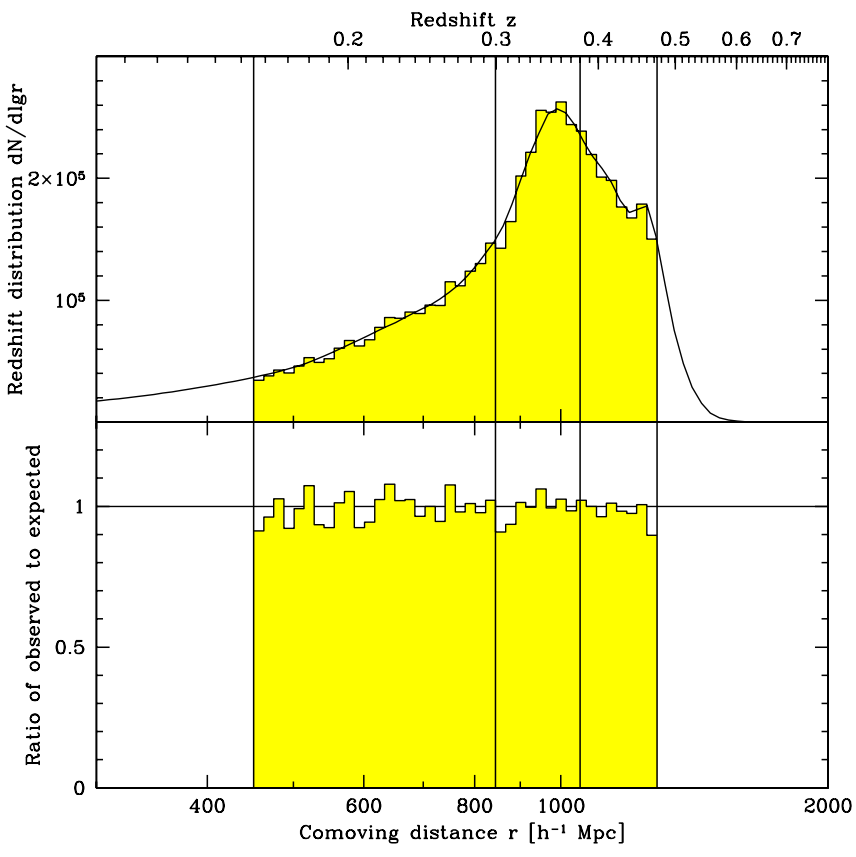

FIG. 1 (color online). The redshift distribution of the luminous red galaxies used is shown as a histogram and compared with the expected distribution in the absence of clustering, $\ln 10 \times$ $\int \bar{n}(r) r^{3} d \Omega$ (solid curve) in comoving coordinates assuming a flat $\Omega_{\Lambda}=0.75$ cosmology. The bottom panel shows the ratio of observed and expected distributions. The four vertical lines delimit the NEAR, MID, and FAR samples.

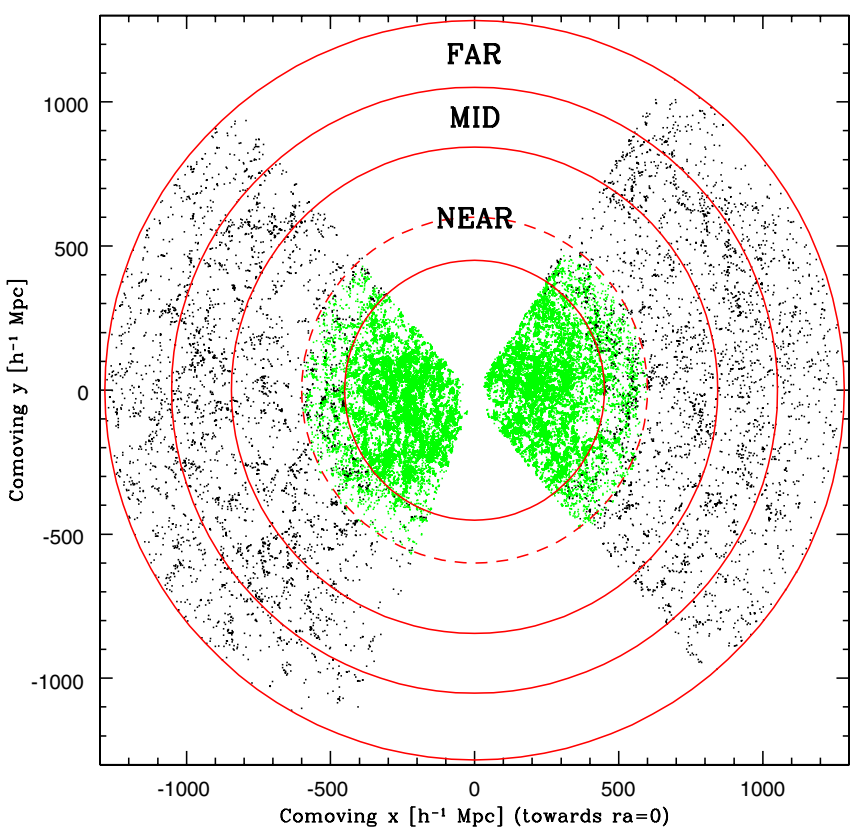

FIG. 2 (color online). The distribution of the 6476 LRGs (black) and 32417 main galaxies (green/gray) that are within $1.25^{\circ}$ of the Equatorial plane. The solid circles indicate the boundaries of our NEAR, MID, and FAR subsamples. The safe13 main galaxy sample analyzed here and in [28] is more local, extending out only to $600 \mathrm{~h}^{-1} \mathrm{Mpc}$ (dashed circle). 


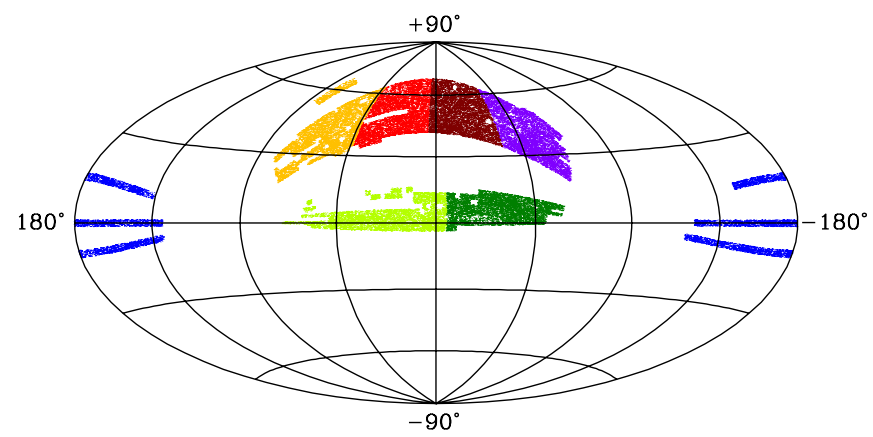

FIG. 3 (color online). The angular distribution of our LRGs is shown in Hammer-Aitoff projection in celestial coordinates, with the seven colors/greys indicating the seven angular subsamples that we analyze.

For systematics testing and numerical purposes, we also analyze a variety of subvolumes in the LRG sample. We split the sample into three radial slices, labeled NEAR $(0.155<z<0.300)$, MID $(0.300<z<0.380)$, and FAR $(0.380<z<0.474)$, containing roughly equal numbers of galaxies, as illustrated in Fig. 2. Their galaxy-weighted mean redshifts are $0.235,0.342$, and 0.421 , respectively. We also split the sample into the seven angular regions illustrated in Fig. 3, each again containing roughly the same number of galaxies.

It is worth emphasizing that the LRGs constitute a remarkably clean and uniform galaxy sample, containing the same type of galaxy (luminous early-types) at all redshifts. Not only is it nearly complete $(\bar{n}(\hat{\mathbf{r}}) \sim 1$ as mentioned above), but it is close to volume-limited for $z \lesssim 0.38$ [36,49], i.e., for our NEAR and MID slices.

\section{POWER SPECTRUM MEASUREMENTS}

We measure the power spectrum of our various samples using the PKL method described in [28]. We follow the procedure of [28] exactly, with some additional numerical improvements described in Appendix A, so we merely summarize the process very briefly here. The first step is to adjust the galaxy redshifts slightly to compress so-called fingers-of-god (FOGs), virialized galaxy clusters that appear elongated along the line-of-sight in redshift space; we do this with several different thresholds and return to how this affects the results in Sec. IV F 2. The LRGs are not just brightest cluster galaxies; about $20 \%$ of them appear to reside in a dark matter halo with one or more other LRG's. The second step is to expand the three-dimensional galaxy density field in $N$ three-dimensional functions termed PKL-eigenmodes, whose variance and covariance retain essentially all the information about the $k<0.2 h / \mathrm{Mpc}$ power spectrum from the galaxy catalog. We use $N=$ 42000 modes for the LRG sample and 4000 modes for the main sample, reflecting their very different effective volumes. The third step is estimating the power spectrum from quadratic combinations of these PKL mode coefficients by a matrix-based process analogous to the standard procedure for measuring $\mathrm{CMB}$ power spectra from pixelized CMB maps. The second and third steps are mathematically straightforward but, as mentioned, numerically demanding for large $N$.

\section{A. Basic results}

The measured real-space power spectra are shown in Fig. 4 for the LRG and MAIN samples and are listed in Table I. When interpreting them, two points should be borne in mind:

(1) The data points (a.k.a. band power measurements) probe a weighted average of the true power spectrum $P(k)$ defined by the window functions shown in Fig. 5. Each point is plotted at the median $k$-value of its window with a horizontal bar ranging from the 20th to the 80th percentile.

(2) The errors on the points, indicated by the vertical bars, are uncorrelated, even though the horizontal bars overlap. Other power spectrum estimation methods (see Appendix A 1) effectively produce a smoothed version of what we are plotting, with error bars that are smaller but highly correlated.

Our Fourier convention is such that the dimensionless power $\Delta^{2}$ of [77] is given by $\Delta^{2}(k)=4 \pi(k / 2 \pi)^{3} P(k)$.

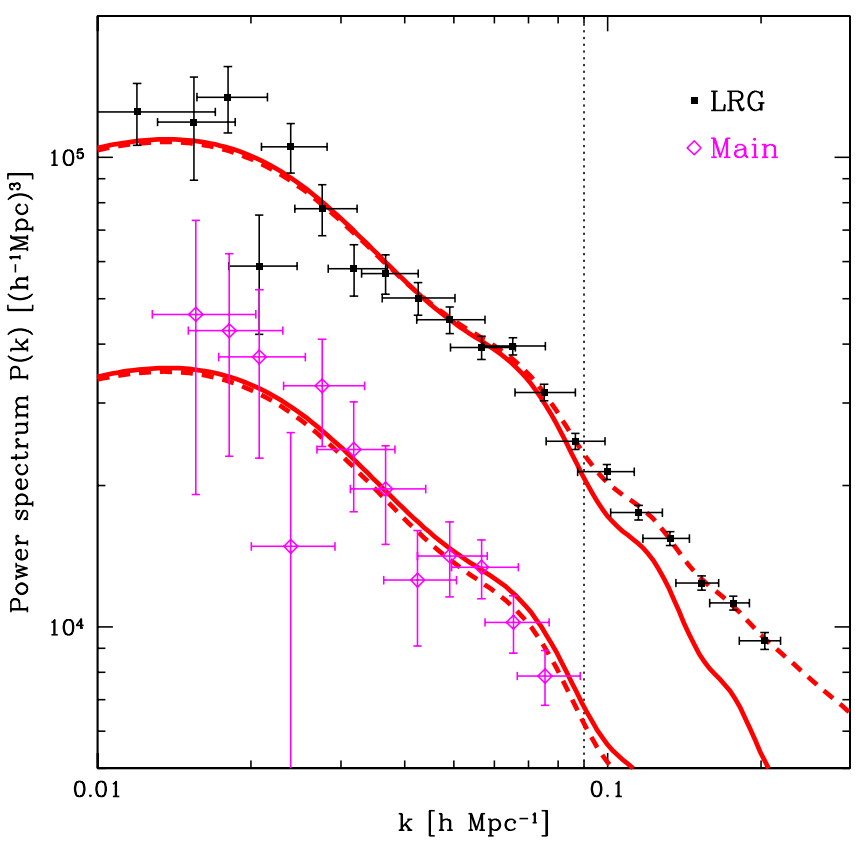

FIG. 4 (color online). Measured power spectra for the full LRG and main galaxy samples. Errors are uncorrelated and full window functions are shown in Fig. 5. The solid curves correspond to the linear-theory $\Lambda \mathrm{CDM}$ fits to WMAP3 alone from Table 5 of [7], normalized to galaxy bias $b=1.9$ (top) and $b=1.1$ (bottom) relative to the $z=0$ matter power. The dashed curves include the nonlinear correction of [29] for $A=1.4$, with $Q_{\mathrm{nl}}=30$ for the LRGs and $Q_{\mathrm{nl}}=4.6$ for the main galaxies; see Eq. (4). The onset of nonlinear corrections is clearly visible for $k \gtrsim 0.09 h / \mathrm{Mpc}$ (vertical line). 
TABLE I. The real-space galaxy power spectrum $P_{\mathrm{g}}(k)$ in units of $\left(h^{-1} \mathrm{Mpc}\right)^{3}$ measured from the LRG sample. The errors on $P_{\mathrm{g}}$ are $1 \sigma$, uncorrelated between bands. The $k$-column gives the median of the window function and its 20th and 80th percentiles; the exact window functions from http://space.mit.edu/home/tegmark/sdss.html (see Fig. 5) should be used for any quantitative analysis. Nonlinear modeling is definitely required if the six measurements on the smallest scales (below the line) are used for model fitting. These error bars do not include an overall calibration uncertainty of $3 \%(1 \sigma)$ related to redshiftspace distortions (see Appendix A 3).

\begin{tabular}{cc}
\hline \hline$k[h / \mathrm{Mpc}]$ & Power $P_{\mathrm{g}}$ \\
\hline $0.012_{-0.004}^{+0.005}$ & $124884 \pm 18775$ \\
$0.015_{-0.003}^{+0.002}$ & $118814 \pm 29400$ \\
$0.018_{-0.004}^{+0.002}$ & $134291 \pm 21638$ \\
$0.021_{-0.003}^{+0.004}$ & $58644 \pm 16647$ \\
$0.024_{-0.003}^{+0.004}$ & $105253 \pm 12736$ \\
$0.028_{-0.003}^{+0.005}$ & $77699 \pm 9666$ \\
$0.032_{-0.003}^{+0.005}$ & $57870 \pm 7264$ \\
$0.037_{-0.006}^{+0.006}$ & $56516 \pm 5466$ \\
$0.043_{-0.008}^{+0.006}$ & $50125 \pm 3991$ \\
$0.049_{-0.008}^{+0.008}$ & $45076 \pm 2956$ \\
$0.057_{-0.009}^{+0.009}$ & $39339 \pm 2214$ \\
$0.065_{-0.008}^{+0.010}$ & $39609 \pm 1679$ \\
$0.075_{-0.009}^{+0.011}$ & $31566 \pm 1284$ \\
$0.087_{-0.011}^{+0.012}$ & $24837 \pm 991$ \\
$0.100_{-0.012}^{+0.013}$ & $21390 \pm 778$ \\
$0.115_{-0.013}^{+0.013}$ & $17507 \pm 629$ \\
$0.133_{-0.012}^{+0.015}$ & $15421 \pm 516$ \\
$0.153_{-0.017}^{+0.012}$ & $12399 \pm 430$ \\
$0.177_{-0.018}^{+0.013}$ & $11237 \pm 382$ \\
$0.203_{-0.022}^{+0.015}$ & $9345 \pm 384$ \\
\hline \hline &
\end{tabular}

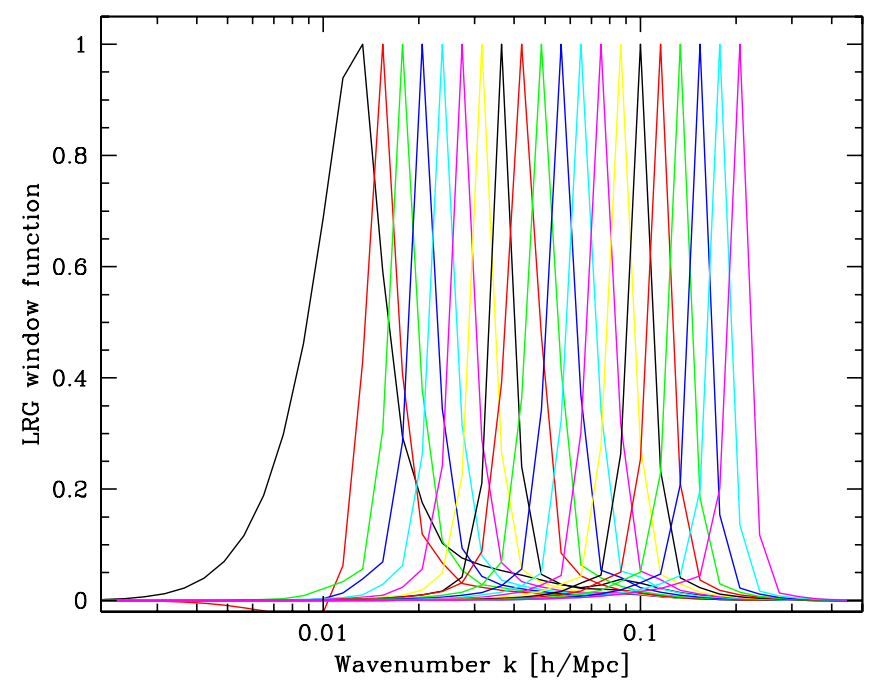

FIG. 5 (color online). The window functions corresponding to the LRG band powers in Fig. 4 are plotted, normalized to have unit peak height. Each window function typically peaks at the scale $k$ that the corresponding band power estimator was designed to probe.
Before using these measurements to constrain cosmological models, one faces important issues regarding their interpretation, related to evolution, nonlinearities and systematics.

\section{B. Clustering evolution}

The standard theoretical expectation is for matter clustering to grow over time and for bias (the relative clustering of galaxies and matter) to decrease over time [78-80] for a given class of galaxies. Bias is also luminosity-dependent, which would be expected to affect the FAR sample but not the MID and NEAR samples (which are effectively volume limited with a $z$-independent mix of galaxy luminosities [49]). Since the galaxy clustering amplitude is the product of these two factors, matter clustering and bias, it could therefore in principle either increase or decrease across the redshift range $0.155<z<0.474$ of the LRG sample. We quantify this empirically by measuring the power spectra of the NEAR, MID and FAR LRG subsamples. The results are plotted in Fig. 6 and show no evidence for evolution of the large-scale galaxy $(k \lesssim 0.1 \mathrm{~h} / \mathrm{Mpc})$ power spectrum in either shape or amplitude. To better quantify this, we fit the WMAP-only best-fit $\Lambda$ CDM model from Table 5 of [7] (solid line in Fig. 6) to our power spectra, by scaling its predicted $z=0$ matter power spectrum by $b^{2}$ for a constant bias factor $b$, using only the 14 data points that are essentially in the linear regime, leftward of the dotted vertical line $k=0.09 h / \mathrm{Mpc}$. For the NEAR, MID, and FAR subsamples, this gives best-fit bias factors $b \approx 1.95$,

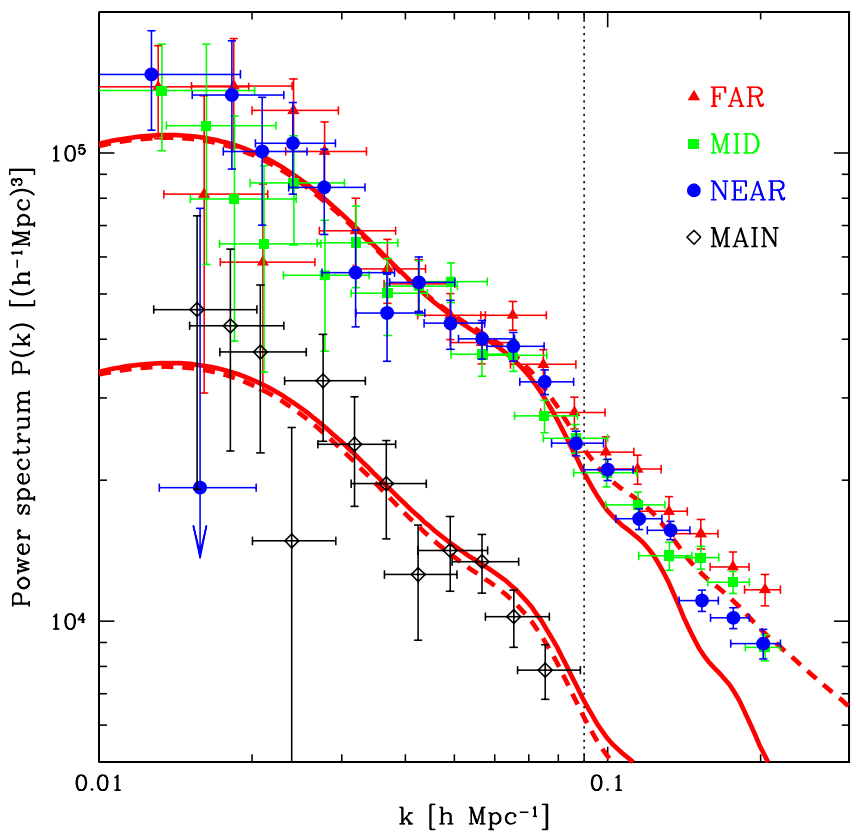

FIG. 6 (color online). Same as Fig. 4, but showing the NEAR (circles), MID (squares) and FAR (triangles) LRG subsamples. On linear scales, they are all well fit by the WMAP3 model with the same clustering amplitude, and there is no sign of clustering evolution. 
1.91, and 2.02, respectively. The fits are all good, giving $\chi^{2} \approx 10.3,11.2$, and 15.9 for the three cases, in agreement with the expectation $\chi^{2}=13 \pm \sqrt{2 \times 13} \approx 13 \pm 5$ and consistent with the linear-theory prediction that the largescale LRG power spectrum should not change its shape over time, merely (perhaps) its amplitude.

The overall amplitude of the LRG power spectrum is constant within the errors over this redshift range, in good agreement with the results of $[41,56]$ at the corresponding mean redshifts. Relative to the NEAR sample, the clustering amplitude is $2.4 \% \pm 3 \%$ lower in MID and 3.5\% $\pm 3 \%$ higher in FAR. In other words, in what appears to be a numerical coincidence, the growth over time in the matter power spectrum is approximately canceled by a drop in the bias factor to within our measurement uncertainty. For a flat $\Omega_{m}=0.25 \Lambda \mathrm{CDM}$ model, the matter clustering grows by about $10 \%$ from the FAR to NEAR sample mean redshifts, so this suggests that the bias drops by a similar factor. For a galaxy population evolving passively, under the influence of gravity alone [78,79], $b$ would be expected to drop by about $5 \%$ over this redshift range; a slight additional drop could be caused by luminosity-dependent bias coupling to the slight change in the luminosity function for the FAR sample, which is not volume limited.

This cancellation of LRG clustering evolution is a fortuitous coincidence that simplifies our analysis: we can pool all our LRGs and measure a single power spectrum for this single sample. It is not a particularly surprising result: many authors have found that the galaxy clustering strength is essentially independent of redshift, even to redshifts $z>3$ [81], and even the effect that is partly canceled (the expected $10 \%$ growth in matter clustering) is small, because of the limited redshift range probed.

\section{Redshift-space distortions}

As described in detail in [28], an intermediate step in our PKL-method is measuring three separate power spectra, $P_{\mathrm{gg}}(k), P_{\mathrm{gv}}(k)$, and $P_{\mathrm{vv}}(k)$, which encode clustering anisotropies due to redshift-space distortions. Here "velocity" refers to the negative of the peculiar velocity divergence. Specifically, $P_{\mathrm{gg}}(k)$ and $P_{\mathrm{vv}}(k)$ are the power spectra of the galaxy density and velocity fields, respectively, whereas $P_{\mathrm{gv}}(k)$ is the cross-power between galaxies and velocity, all defined in real space rather than redshift space.

In linear perturbation theory, these three power spectra are related by [82]

$$
\begin{gathered}
P_{\mathrm{gv}}(k)=\beta r_{\mathrm{gv}} P_{\mathrm{gg}}(k), \\
P_{\mathrm{vv}}(k)=\beta^{2} P_{\mathrm{gg}}(k),
\end{gathered}
$$

where $\beta \equiv f / b, b$ is the bias factor, $r_{\mathrm{gv}}$ is the dimensionless correlation coefficient between the galaxy and matter density fields $[79,83,84]$, and $f \approx \Omega_{m}^{0.6}$ is the dimensionless linear growth rate for linear density fluctuations. (When computing $f$ below, we use the more accurate approximation of [85].)
The LRG power spectrum $P(k)$ tabulated and plotted above is a minimum-variance estimator of $P_{\mathrm{gg}}(k)$ that linearly combines the $P_{\mathrm{gg}}(k), P_{\mathrm{gv}}(k)$ and $P_{\mathrm{vv}}(k)$ estimators as described in [28] and Appendix A 3, effectively marginalizing over the redshift-space distortion parameters $\beta$ and $r_{\mathrm{gv}}$. As shown in Appendix A 3, this linear combination is roughly proportional to the angle-averaged (monopole) redshift-space galaxy power spectrum, so for the purposes of the nonlinear modeling in the next section, the reader may think of our measured $P(k)$ as essentially a rescaled version of the redshift-space power spectrum. However, unlike the redshift-space power spectrum measured with the FKP and FFT methods (Appendix A 1), our measured $P(k)$ is unbiased on large scales. This is because linear redshift distortions are treated exactly, without resorting to the small-angle approximation, and account is taken of the fact that the anisotropic survey geometry can skew the relative abundance of galaxy pairs around a single point as a function of angle to the line of sight.

The information about anisotropic clustering that is discarded in our estimation of $P(k)$ allows us to measure $\beta$ and perform a powerful consistency test. Figure 8 shows the joint constraints on $\beta$ and $r_{\mathrm{gv}}$ from fitting Eqs. (2) and (3) to the $0.01 h / \mathrm{Mpc} \leq k \leq 0.09 h / \mathrm{Mpc}$ LRG data, using the best-fit WMAP3 model from Fig. 4 for $P_{\mathrm{gg}}(k)$ and marginalizing over its amplitude. The data are seen to favor $r_{\mathrm{gv}} \approx 1$ in good agreement with prior work [86,87]. Assuming $r_{\mathrm{gv}}=1$ (that galaxy density linearly traces matter density on these large scales) gives the measurement $\beta=0.309 \pm 0.035(1 \sigma)$. This measurement is rather robust to changing the FOG-compression threshold by a notch (Sec. IVF2) or slightly altering the maximum $k$-band included, both of which affect the central value by of order 0.01 . As a cross-check, we can compute $\beta=$ $f\left(\Omega_{m}, \Omega_{\Lambda}\right) / b$ at the median survey redshift based on our multiparameter analysis presented in Sec. IV, which for our vanilla class of models gives $\beta=0.280 \pm 0.014$ (marked with a diamond in Fig. 8). ${ }^{1}$ That these two $\beta$-measurements agree within $1 \sigma$ is highly nontrivial, since the second $\beta$-measurement makes no use whatsoever of redshift-space distortions, but rather extracts $b$ from the ratio of LRG power to CMB power, and determines $\Omega_{m}$ from CMB and LRG power spectrum shapes.

\section{Nonlinear modeling}

Above we saw that our $k<0.09 h / \mathrm{Mpc}$ measurements of the LRG power spectrum were well fit by the lineartheory matter power spectrum predicted by WMAP3. In contrast, Figs. 4, 6, and 7 show clear departures from the linear-theory prediction on smaller scales. There are sev-

\footnotetext{
${ }^{1}$ Here $\beta=f\left(\Omega_{m}, \Omega_{\Lambda}\right) / b$ is computed with $\Omega_{m}, \Omega_{\Lambda}$ and $b$ evaluated at the median redshift $z=0.35$, when $b=2.25 \pm$ 0.08 , taking into account linear growth of matter clustering between then and now.
} 
eral reasons for this that have been extensively studied in the literature:

(1) Nonlinear evolution alters the broad shape of the matter power spectrum on small scales.

(2) Nonlinear evolution washes out baryon wiggles on small scales.

(3) The power spectrum of the dark matter halos in which the galaxies reside differs from that of the underlying matter power spectrum in both amplitude and shape, causing bias.

(4) Multiple galaxies can share the same dark matter halo, enhancing small-scale bias.

We fit these complications using a model involving the three "nuisance parameters" $\left(b, Q_{\mathrm{nl}}, k_{*}\right)$ as illustrated in Fig. 9. Following $[29,88]$, we model our measured galaxy power spectrum as

$$
P_{\mathrm{g}}(k)=P_{\text {dewiggled }}(k) b^{2} \frac{1+Q_{\mathrm{nl}} k^{2}}{1+1.4 k},
$$

where the first factor on the right hand side accounts for the nonlinear suppression of baryon wiggles and the last factor accounts for a combination of the nonlinear change of the global matter power spectrum shape and scale-dependent bias of the galaxies relative to the dark matter. For $P_{\text {dewiggled }}(k)$ we adopt the prescription [88]

$$
P_{\text {dewiggled }}(k)=W(k) P(k)+[1-W(k)] P_{\text {nowiggle }}(k),
$$

where $W(k) \equiv e^{-\left(k / k_{*}\right)^{2} / 2}$ and $P_{\text {nowiggle }}(k)$ denotes the "no wiggle" power spectrum defined in [89] and illustrated in Fig. 9. In other words, $P_{\text {dewiggled }}(k)$ is simply a weighted average of the linear power spectrum and the wiggle-free

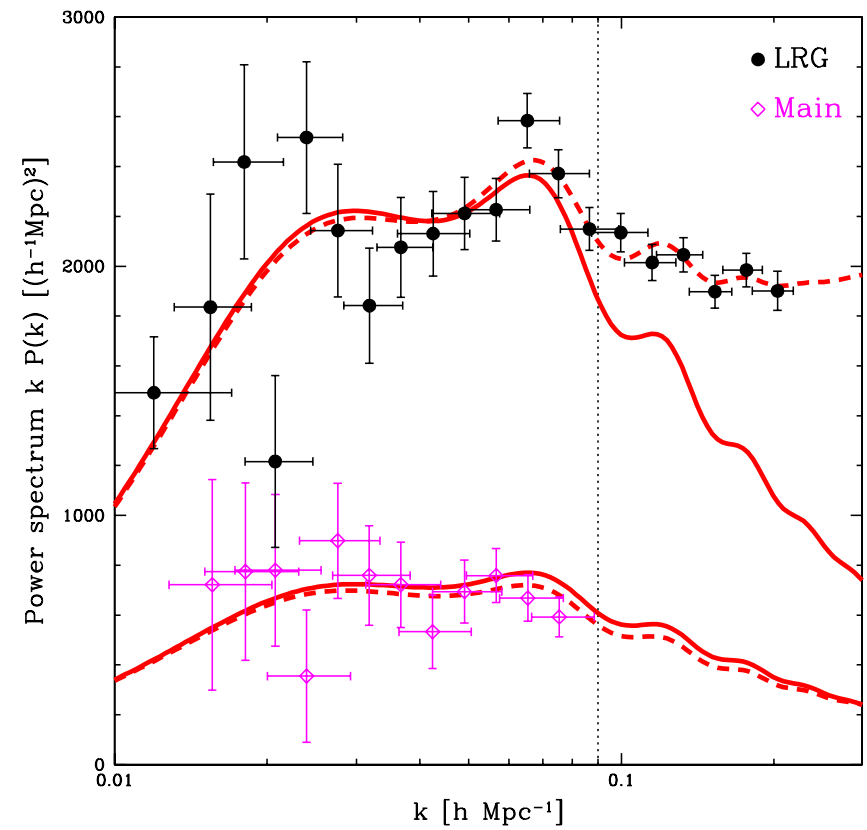

FIG. 7 (color online). Same as Fig. 4, but multiplied by $k$ and plotted with a linear vertical axis to more clearly illustrate departures from a simple power law.

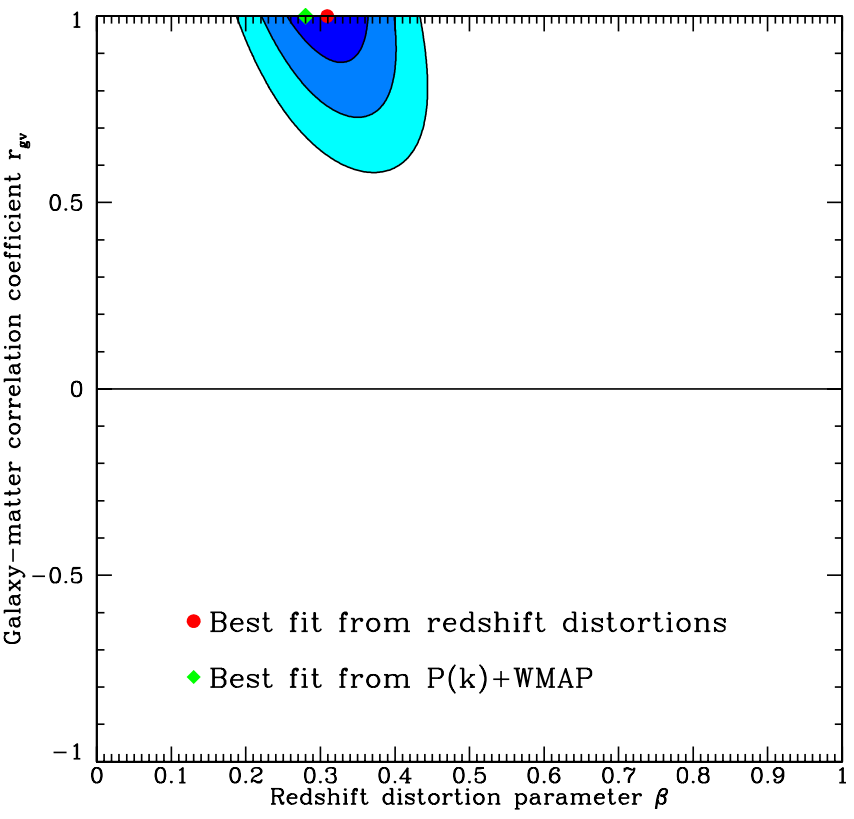

FIG. 8 (color online). Constraints on the redshift-space distortion parameters $\beta$ and $r_{\mathrm{gv}}$. The contours show the 1,2 , and $3 \sigma$ constraints from the observed LRG clustering anisotropy, with the circular dot indicating the best-fit values. The diamond shows the completely independent $\beta$-estimate inferred from our analysis of the WMAP3 and LRG power spectra (it puts no constraints on $r_{\mathrm{gv}}$, but has been plotted at $r_{\mathrm{gv}}=1$ ).

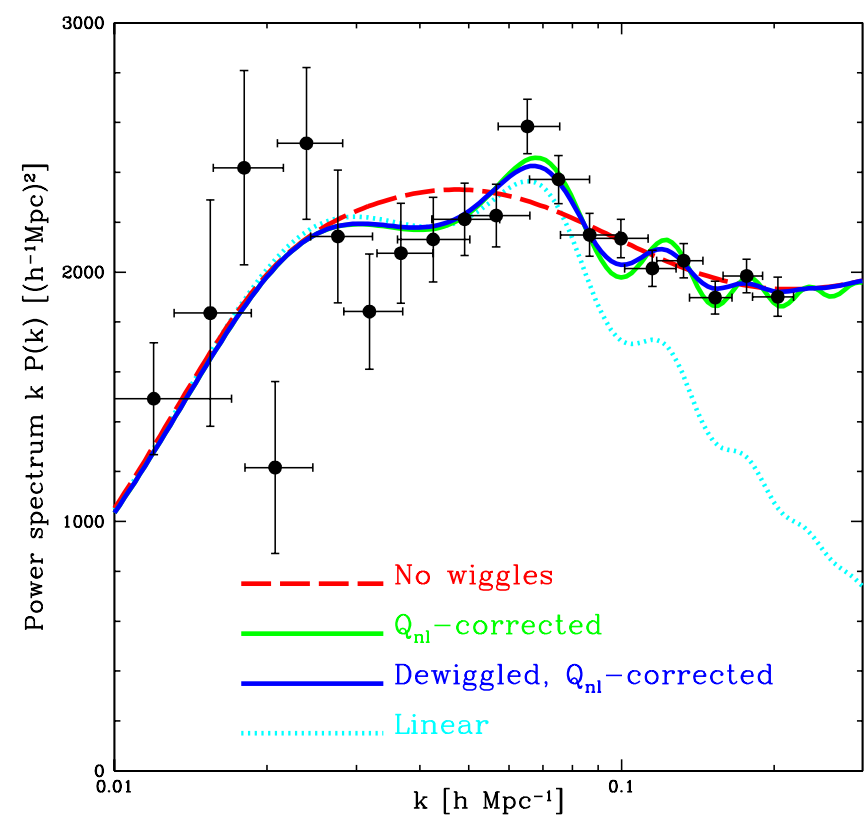

FIG. 9 (color online). Power spectrum modeling. The best-fit WMAP3 model from Table 5 of [7] is shown with a linear bias $b=1.89$ (dotted curve), after applying the nonlinear bias correction with $Q=31$ (the more wiggly solid curve), and after also applying the wiggle suppression of [88] (the less wiggly solid curve), which has no effect on very large scales and asymptotes to the "no wiggle" spectrum of [89] (dashed curve) on very small scales. The data points are the LRG measurements from Fig. 7. 
version thereof. Since the $k$-dependent weight $W(k)$ transitions from 1 for $k \ll k_{*}$ to 0 for $k \gg k_{*}$, Eq. (5) retains wiggles on large scales and gradually fades them out beginning around $k=k_{*}$. Inspired by [88], we define the wiggle suppression scale $k_{*} \equiv 1 / \sigma$, where $\sigma \equiv$ $\sigma_{\perp}^{2 / 3} \sigma_{\|}^{1 / 3}\left(A_{s} / 0.6841\right)^{1 / 2}$ and $\sigma_{\perp}$ and $\sigma_{\|}$are given by equations (12) and (13) in [88] based on fits to cosmological $\mathrm{N}$-body simulations. The expression in parenthesis is an amplitude scaling factor that equals unity for the best-fit WMAP3 normalization $A_{s}=0.6841$ of [7]. Essentially, $\sigma$ is the characteristic peculiar-velocity-induced displacement of galaxies that causes the wiggle suppression; [88] define it for a fixed power spectrum normalization, and it scales linearly with fluctuation amplitude, i.e., $\propto A_{s}^{1 / 2}$. For the cosmological parameter range allowed by WMAP3, we find that $k_{*} \sim 0.1 h / \mathrm{Mpc}$, with a rather rather weak dependence on cosmological parameters (mainly $\Omega_{m}$ and $A_{s}$ ).

The simulations and analytic modeling described by [29] suggest that the $Q_{\mathrm{nl}}$-prescription given by Eq. (4) accurately captures the scale-dependent bias of galaxy populations on the scales that we are interested in, though they examined samples less strongly biased than the LRGs considered here. To verify the applicability of this prescription for LRGs in combination with our dewiggling model, we reanalyze the $51 n$-body simulations described in [90], each of which uses a $512 h^{-1} \mathrm{Mpc}$ box with $256^{3}$ particles and WMAP1 parameters. Figure 10 compares these simulation results with our nonlinear modeling prediction defined by Eqs. (4) and (5) for $b=2.02, Q_{\mathrm{nl}}=$ 27.0, showing excellent agreement (at the $1 \%$ level) for $k \lesssim 0.4 h / \mathrm{Mpc}$. Choosing a $k_{*}$ very different from $0.1 \mathrm{~h} / \mathrm{Mpc}$ causes $5 \%$ wiggles to appear in the residuals because of a over- or under-suppression of the baryon oscillations. These simulations are likely to be underresolved and the LRG halo prescription used (one LRG for each halo above a threshold mass of $8 \times 10^{12} M_{\odot}$ ) is clearly overly simplistic, so the true value of $Q_{\mathrm{nl}}$ that best describes LRGs could be somewhat different. Nonetheless, this test provides encouraging evidence that Eq. (4) is accurate in combination with Eq. (5) and that our $Q=$ $30 \pm 4$ measurement from Table II is plausible. Further corroboration is provided by the results in [41] using the millennium simulation [97]. Here LRG type galaxies were simulated and selected in an arguably more realistic way, yet giving results nicely consistent with Fig. 10, with a best-fit value $Q_{\mathrm{nl}} \approx 24$. (We will see in Sec. IVF that FOG-compression can readily account for these slight differences in $Q_{\mathrm{nl}}$-value.) A caveat to both of these simulation tests is that they were performed in real space, and our procedure for measuring $P_{\mathrm{g}}(k)$ reconstructs the realspace power spectrum exactly only in the linear regime [28]. Thus, these results should be viewed as encouraging but preliminary, and more work is needed to establish the validity of the nonlinear modeling beyond $k \geq 0.1 \mathrm{~h} / \mathrm{Mpc}$;

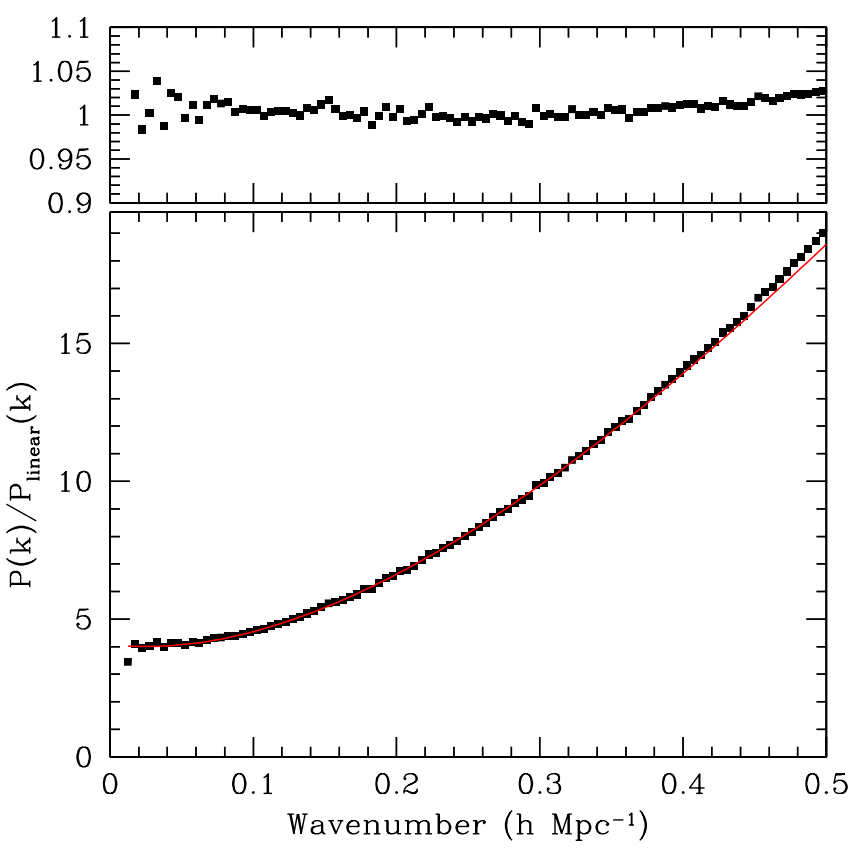

FIG. 10 (color online). The points in the bottom panel show the ratio of the real-space power spectrum from 51 averaged $n$-body simulations (see text) to the linear power spectrum dewiggled with $k_{*}=0.1 \mathrm{~h} / \mathrm{Mpc}$. Here LRGs were operationally defined as halos with mass exceeding $8 \times 10^{12} M_{\odot}$, corresponding to at least ten simulation particles. The solid curve shows the prediction from Eq. (4) with $b=2.02, Q_{\mathrm{nl}}=27$, seen to be an excellent fit for $k \lesssim 0.4 h / \mathrm{Mpc}$. The top panel shows the ratio of the simulation result to this fit. Although the simulation specifications and the LRG identification prescription can clearly be improved, they constitute the first and only that we tried, and were in no way adjusted to try to fit our $Q_{\mathrm{nl}}=30 \pm 4$ measurement from Table II. This agreement suggests that our use of Eqs. (4) and (5) to model nonlinearities is reasonable and that our measured $Q_{\mathrm{nl}}$-value is plausible.

for up-to-date discussions and a variety of ideas for paths forward, see, e.g., [98-101].

In addition to this simulation-based theoretical evidence that our nonlinear modeling method is accurate, we have encouraging empirical evidence: Fig. 9 shows an excellent fit to our measurements. Fitting the best-fit WMAP3 model from [32] to our first 20 data points (which extend out to $k=0.2 h / \mathrm{Mpc})$ by varying $\left(b, Q_{\mathrm{nl}}\right)$ gives $\chi^{2}=19.2$ for $20-2=18$ degrees of freedom, where the expected $1 \sigma$ range is $\chi^{2}=18 \pm(2 \times 18)^{1 / 2}=18 \pm 6$, so the fit is excellent. Moreover, Figs. 7 and 9 show that that main outliers are on large and highly linear scales, not on the smaller scales where our nonlinear modeling has an effect.

The signature of baryons is clearly seen in the measured power spectrum. If we repeat this fit with baryons replaced by dark matter, $\chi^{2}$ increases by 8.8 , corresponding to a baryon detection at $3.0 \sigma$ (99.7\% significance). Much of this signature lies in the acoustic oscillations: if we instead repeat the fit with $k_{*}=0$, corresponding to fully removing the wiggles, $\chi^{2}$ increases by an amount corresponding to a 
TABLE II. Cosmological parameters measured from WMAP and SDSS LRG data with the Occam's razor approach described in the text: the constraint on each quantity is marginalized over all other parameters in the vanilla set $\left(\omega_{b}, \omega_{\mathrm{c}}, \Omega_{\Lambda}, A_{s}, n_{s}, \tau, b, Q_{\mathrm{nl}}\right)$. Error bars are $1 \sigma$.

\begin{tabular}{|c|c|c|c|}
\hline Paramet & Value & Meaning & Definition \\
\hline \multicolumn{4}{|c|}{ Matter budget parameters: } \\
\hline$\Omega_{\text {tot }}$ & $1.003_{-0.009}^{+0.010}$ & Total density/critical density & $\Omega_{\mathrm{tot}}=\Omega_{m}+\Omega_{\Lambda}=1-\Omega_{k}$ \\
\hline$\Omega_{\Lambda}$ & $0.761_{-0.018}^{+0.017}$ & Dark energy density parameter & $\Omega_{\Lambda} \approx h^{-2} \rho_{\Lambda}\left(1.88 \times 10^{-26} \mathrm{~kg} / \mathrm{m}^{3}\right)$ \\
\hline$\omega_{b}$ & $0.0222_{-0.0007}^{+0.0007}$ & Baryon density & $\omega_{b}=\Omega_{b} h^{2} \approx \rho_{b} /\left(1.88 \times 10^{-26} \mathrm{~kg} / \mathrm{m}^{3}\right)$ \\
\hline$\omega_{c}$ & $0.1050_{-0.0040}^{+0.0041}$ & Cold dark matter density & $\omega_{\mathrm{c}}=\Omega_{\mathrm{c}} h^{2} \approx \rho_{c} /\left(1.88 \times 10^{-26} \mathrm{~kg} / \mathrm{m}^{3}\right)$ \\
\hline$\omega_{\nu}$ & $<0.010(95 \%)$ & Massive neutrino density & $\omega_{\nu}=\Omega_{\nu} h^{2} \approx \rho_{\nu} /\left(1.88 \times 10^{-26} \mathrm{~kg} / \mathrm{m}^{3}\right)$ \\
\hline$w$ & $-0.941_{-0.101}^{+0.087}$ & Dark energy equation of state & $p_{\Lambda} / \rho_{\Lambda}$ (approximated as constant) \\
\hline \multicolumn{4}{|c|}{ Seed fluctuation parameters: } \\
\hline$A_{s}$ & $0.690_{-0.044}^{+0.045}$ & Scalar fluctuation amplitude & Primordial scalar power at $k=0.05 / \mathrm{Mpc}$ \\
\hline$r$ & $<0.30(95 \%)$ & Tensor-to-scalar ratio & Tensor-to-scalar power ratio at $k=0.05 / \mathrm{Mpc}$ \\
\hline$n_{s}$ & $0.953_{-0.016}^{+0.016}$ & Scalar spectral index & Primordial spectral index at $k=0.05 / \mathrm{Mpc}$ \\
\hline$n_{t}+1$ & $0.9861_{-0.0142}^{+0.0096}$ & Tensor spectral index & $n_{t}=-r / 8$ assumed \\
\hline$\alpha$ & $-0.040_{-0.027}^{+0.027}$ & Running of spectral index & $\alpha=d n_{s} / d \ln k$ (approximated as constant) \\
\hline \multicolumn{4}{|c|}{ Nuisance parameters: } \\
\hline$\tau$ & $0.087_{-0.030}^{+0.028}$ & Reionization optical depth & \\
\hline$b$ & $1.896_{-0.069}^{+0.074}$ & Galaxy bias factor & $b=\left[P_{\text {galaxy }}(k) / P(k)\right]^{1 / 2}$ on large scales, where $P(k)$ refers to today. \\
\hline$Q_{\mathrm{nl}}$ & $30.3_{-4.1}^{+4.4}$ & Nonlinear correction parameter [29] & $P_{\mathrm{g}}(k)=P_{\text {dewiggled }}(k) b^{2}\left(1+Q_{\mathrm{nl}} k^{2}\right) /(1+1.7 k)$ \\
\hline \multicolumn{4}{|c|}{ Other popular parameters (determined by those above): } \\
\hline$h$ & $0.730_{-0.019}^{+0.019}$ & Hubble parameter & $h=\sqrt{\left(\omega_{b}+\omega_{\mathrm{c}}+\omega_{\nu}\right) /\left(\Omega_{\mathrm{tot}}-\Omega_{\Lambda}\right)}$ \\
\hline$\Omega_{m}$ & $0.239_{-0.017}^{+0.018}$ & Matter density/critical density & $\Omega_{m}=\Omega_{\mathrm{tot}}-\Omega_{\Lambda}$ \\
\hline$\Omega_{b}$ & $0.0416_{-0.0018}^{+0.0019}$ & Baryon density/critical density & $\Omega_{b}=\omega_{b} / h^{2}$ \\
\hline$\Omega_{c}$ & $0.197_{-0.015}^{+0.016}$ & CDM density/critical density & $\Omega_{\mathrm{c}}=\omega_{\mathrm{c}} / h^{2}$ \\
\hline$\Omega_{\nu}$ & $<0.024(95 \%)$ & Neutrino density/critical density & $\Omega_{\nu}=\omega_{\nu} / h^{2}$ \\
\hline$\Omega_{k}$ & $-0.0030_{-0.0102}^{+0.0095}$ & Spatial curvature & $\Omega_{k}=1-\Omega_{\mathrm{tot}}$ \\
\hline$\omega_{m}$ & $0.1272_{-0.0043}^{+0.0044}$ & Matter density & $\omega_{\mathrm{m}}=\omega_{b}+\omega_{\mathrm{c}}+\omega_{\nu}=\Omega_{m} h^{2}$ \\
\hline$f_{\nu}$ & $<0.090(95 \%)$ & Dark matter neutrino fraction & $f_{\nu}=\rho_{\nu} / \rho_{d}$ \\
\hline$A_{t}$ & $<0.21(95 \%)$ & Tensor fluctuation amplitude & $A_{t}=r A_{s}$ \\
\hline$M_{\nu}$ & $<0.94(95 \%) \mathrm{eV}$ & Sum of neutrino masses & $M_{\nu} \approx(94.4 \mathrm{eV}) \times \omega_{\nu}[91]$ \\
\hline$A_{.002}$ & $0.801_{-0.043}^{+0.042}$ & WMAP3 normalization parameter & $A_{s}$ scaled to $k=0.002 / \mathrm{Mpc}: A_{.002}=25^{1-n_{s}} A_{s}$ if $\alpha=0$ \\
\hline$r_{.002}$ & $<0.33(95 \%)$ & Tensor-to-scalar ratio (WMAP3) & Tensor-to-scalar power ratio at $k=0.002 / \mathrm{Mpc}$ \\
\hline$\sigma_{8}$ & $0.756_{-0.035}^{+0.035}$ & Density fluctuation amplitude & $\sigma_{8}=\left\{4 \pi \int_{0}^{\infty}\left[\frac{3}{x^{3}}(\sin x-x \cos x)\right]^{2} P(k) \frac{k^{2} d k}{(2 \pi)^{3}}\right\}^{1 / 2}, x \equiv k \times 8 h^{-1} \mathrm{Mpc}$ \\
\hline$\sigma_{8} \Omega_{m}^{0.6}$ & $0.320_{-0.023}^{+0.024}$ & Velocity fluctuation amplitude & \\
\hline \multicolumn{4}{|c|}{ Cosmic history parameters: } \\
\hline$z_{\mathrm{eq}}$ & $3057_{-102}^{+105}$ & Matter-radiation Equality redshift & $z_{\mathrm{eq}} \approx 24074 \omega_{\mathrm{m}}-1$ \\
\hline$z_{\text {rec }}$ & $1090.25_{-0.91}^{+0.93}$ & Recombination redshift & $z_{\text {rec }}\left(\omega_{\mathrm{m}}, \omega_{b}\right)$ given by eq. (18) of [92] \\
\hline$z_{\text {ion }}$ & $11.1_{-2.7}^{+2.2}$ & Reionization redshift (abrupt) & $z_{\text {ion }} \approx 92\left(0.03 h \tau / \omega_{b}\right)^{2 / 3} \Omega_{m}^{1 / 3}$ (assuming abrupt reionization; [93]) \\
\hline$z_{\text {acc }}$ & $0.855_{-0.059}^{+0.059}$ & Acceleration redshift & $z_{\mathrm{acc}}=\left[(-3 w-1) \Omega_{\Lambda} / \Omega_{m}\right]^{-1 / 3 w}-1$ if $w<-1 / 3$ \\
\hline$t_{\mathrm{eq}}$ & $0.0634_{-0.0041}^{+0.0045} \mathrm{Myr}$ & Matter-radiation Equality time & $t_{\mathrm{eq}} \approx(9.778 \mathrm{Gyr}) \times h^{-1} \int_{z_{\mathrm{eq}}}^{\infty}\left[H_{0} / H(z)(1+z)\right] d z[91]$ \\
\hline$t_{\text {rec }}$ & $0.3856_{-0.0040}^{+0.0040} \mathrm{Myr}$ & Recombination time & $t_{\mathrm{req}} \approx(9.778 \mathrm{Gyr}) \times h^{-1} \int_{z_{\mathrm{rec}}}^{\infty}\left[H_{0} / H(z)(1+z)\right] d z[91]$ \\
\hline$t_{\text {ion }}$ & $0.43_{-0.10}^{+0.20} \mathrm{Gyr}$ & Reionization time & $t_{\mathrm{ion}} \approx(9.778 \mathrm{Gyr}) \times h^{-1} \int_{z_{\mathrm{ion}}}^{\infty}\left[H_{0} / H(z)(1+z)\right] d z[91]$ \\
\hline$t_{\mathrm{acc}}$ & $6.74_{-0.24}^{+0.25} \mathrm{Gyr}$ & Acceleration time & $t_{\mathrm{acc}} \approx(9.778 \mathrm{Gyr}) \times h^{-1} \int_{z_{\mathrm{acc}}}^{\infty}\left[H_{0} / H(z)(1+z)\right] d z[91]$ \\
\hline & $13.76_{-0.15}^{+0.15} \mathrm{Gyr}$ & Age of Universe now & $t_{\text {now }} \approx(9.778 \mathrm{Gyr}) \times h^{-1} \int_{0}^{\infty}\left[H_{0} / H(z)(1+z)\right] d z[91]$ \\
\hline \multicolumn{4}{|c|}{ Fundamental parameters (independent of observing epoch): } \\
\hline$Q$ & $1.945_{-0.053}^{+0.051} \times 10^{-5}$ & Primordial fluctuation amplitude & $Q=\delta_{h} \approx A_{.002}^{1 / 2} \times 59.2384 \mu \mathrm{K} / T_{\mathrm{CMB}}$ \\
\hline$\kappa$ & $1.3_{-4.3}^{+3.7} \times 10^{-61}$ & Dimensionless spatial curvature [94] & $\kappa=\left(\hbar c / k_{B} T_{\mathrm{CMB}} a\right)^{2} k$ \\
\hline$\rho_{\Lambda}$ & $1.48_{-0.11}^{+0.11} \times 10^{-123} \rho_{\mathrm{Pl}}$ & Dark energy density & $\rho_{\Lambda} \approx h^{2} \Omega_{\Lambda} \times\left(1.88 \times 10^{-26} \mathrm{~kg} / \mathrm{m}^{3}\right)$ \\
\hline$\rho_{\text {halo }}$ & $6.6_{-1.0}^{+1.2} \times 10^{-123} \rho_{\mathrm{Pl}}$ & Halo formation density & $\rho_{\text {halo }}=18 \pi^{2} Q^{3} \xi^{4}$ \\
\hline$\xi$ & $3.26_{-0.11}^{+0.11} \mathrm{eV}$ & Matter mass per photon & $\xi=\rho_{\mathrm{m}} / n_{\gamma}$ \\
\hline$\xi_{b}$ & $0.569_{-0.018}^{+0.018} \mathrm{eV}$ & Baryon mass per photon & $\xi_{\mathrm{b}}=\rho_{\mathrm{b}} / n_{\gamma}$ \\
\hline$\xi_{c}$ & $2.69_{-0.10}^{+0.11} \mathrm{eV}$ & CDM mass per photon & $\xi_{\mathrm{c}}=\rho_{\mathrm{c}} / n_{\gamma}$ \\
\hline$\xi_{\nu}$ & $<0.26(95 \%) \mathrm{eV}$ & Neutrino mass per photon & $\xi_{\nu}=\rho_{\nu} / n_{\gamma}$ \\
\hline$\eta$ & $6.06_{-0.19}^{+0.20} \times 10^{-10}$ & Baryon/photon ratio & $\eta=n_{b} / n_{g}=\xi_{\mathrm{b}} / m_{p}$ \\
\hline$A_{\Lambda}$ & $2077_{-125}^{+135}$ & Expansion during matter domination & $\left(1+z_{\mathrm{eq}}\right)\left(\Omega_{m} / \Omega_{\Lambda}\right)^{1 / 3}[95]$ \\
\hline$\sigma_{\text {gal }}^{*}$ & $0.561_{-0.023}^{+0.024} \times 10^{-3}$ & Seed amplitude on galaxy scale & Like $\sigma_{8}$ but on galactic $\left(M=10^{12} M_{\odot}\right)$ scale early on \\
\hline
\end{tabular}


TABLE II. (Continued)

\begin{tabular}{|c|c|c|c|}
\hline \multicolumn{2}{|c|}{ Parameter Value } & Meaning & Definition \\
\hline \multicolumn{4}{|c|}{ CMB phenomenology parameters: } \\
\hline$A_{\text {peak }}$ & $0.579_{-0.013}^{+0.013}$ & Amplitude on CMB peak scales & $A_{\text {peak }}=A_{s} e^{-2 \tau}$ \\
\hline$A_{\text {pivot }}$ & $0.595_{-0.011}^{+0.012}$ & Amplitude at pivot point & $A_{\text {peak }}$ scaled to $k=0.028 / \mathrm{Mpc}: A_{\text {pivot }}=0.56^{n_{s}-1} A_{\text {peak }}$ if $\alpha=0$ \\
\hline$H_{1}$ & $4.88_{-0.34}^{+0.37}$ & 1st CMB peak ratio & $H_{1}\left(\Omega_{\mathrm{tot}}, \Omega_{\Lambda}, \omega_{b}, \omega_{\mathrm{m}}, w, n_{s}, \tau\right)$ given by [96] \\
\hline $\mathrm{H}_{2}$ & $0.4543_{-0.0051}^{+0.0051}$ & 2nd to 1 st $\mathrm{CMB}$ peak ratio & $\left.H_{2}=\left(0.925 \omega_{\mathrm{m}}^{0.18} 2.4^{n_{s}-1}\right) /\left[1+\left(\omega_{b} / 0.0164\right)^{12 \omega_{\mathrm{m}}^{0.52}}\right)\right]^{0.2}[96]$ \\
\hline $\mathrm{H}_{3}$ & $0.4226_{-0.0086}^{+0.0088}$ & 3rd to 1 st $\mathrm{CMB}$ peak ratio & $\begin{array}{c}H_{3}=2.17\left[1+\left(\omega_{b} / 0.044\right)^{2}\right]^{-1} \omega_{\mathrm{m}}^{0.59} 3.6^{n_{s}-1} / \\
{\left[1+1.63\left(1-\omega_{b} / 0.071\right) \omega_{\mathrm{m}}\right]}\end{array}$ \\
\hline$d_{A}\left(z_{\mathrm{rec}}\right)$ & $14.30_{-0.17}^{+0.17} \mathrm{Gpc}$ & $\begin{array}{l}\text { Comoving angular diameter } \\
\text { distance to } \mathrm{CMB}\end{array}$ & $d_{A}\left(z_{\mathrm{rec}}\right)=\frac{c}{H_{0}} \sinh \left[\Omega_{k}^{1 / 2} \int_{0}^{z_{\mathrm{rec}}}\left[H_{0} / H(z)\right] d z\right] / \Omega_{k}^{1 / 2}[91]$ \\
\hline$r_{s}\left(z_{\text {rec }}\right)$ & $0.1486_{-0.0014}^{+0.0014} \mathrm{Gpc}$ & Comoving sound horizon scale & $r_{\mathrm{s}}\left(\omega_{\mathrm{m}}, \omega_{b}\right)$ given by eq. (22) of [92] \\
\hline$r_{\mathrm{damp}}$ & $0.0672_{-0.0008}^{+0.0009} \mathrm{Gpc}$ & Comoving acoustic damping scale & $r_{\text {damp }}\left(\omega_{\mathrm{m}}, \omega_{b}\right)$ given by Eq. (26) of [92] \\
\hline$\Theta_{s}$ & $0.5918_{-0.0020}^{+0.0020}$ & CMB acoustic angular scale fit (degrees) & $\Theta_{s}\left(\Omega_{\mathrm{tot}}, \Omega_{\Lambda}, w, \omega_{b}, \omega_{\mathrm{m}}\right)$ given by $[96]$ \\
\hline$\ell_{A}$ & $302.2_{-1.0}^{+1.0}$ & CMB acoustic angular scale & $\ell_{A}=\pi d_{A}\left(z_{\mathrm{rec}}\right) / r_{\mathrm{s}}\left(z_{\mathrm{rec}}\right)$ \\
\hline
\end{tabular}

detection of wiggles at $2.3 \sigma$ (98\% significance). The data are not yet sensitive enough to distinguish between the wiggled and dewiggled spectra; dewiggling reduces $\chi^{2}$ by merely 0.04 .

In summary, the fact that LRGs tend to live in high-mass dark matter halos is a double-edged sword: it helps by giving high bias $b \sim 2$ and luminous galaxies observable at great distance, but it also gives a stronger nonlinear correction (higher $Q_{\mathrm{nl}}$ ) that becomes important on larger scales than for typical galaxies. Although Fig. 10 suggests that our nonlinear modeling is highly accurate out to $k=$ $0.4 h / \mathrm{Mpc}$, we retain only measurements with $k \lesssim$ $0.2 \mathrm{~h} / \mathrm{Mpc}$ for our cosmological parameter analysis to be conservative, and plan further work to test the validity of various nonlinear modeling approaches. In Sec. IV F 2, we will see that our data with $0.09 h / \mathrm{Mpc}<k \lesssim 0.2 h / \mathrm{Mpc}$, where nonlinear effects are clearly visible, allow us to constrain the nuisance parameter $Q_{\mathrm{nl}}$ without significantly improving our constraints on cosmological parameters. In other words, the cosmological constraints that we will report below are quite insensitive to our nonlinear modeling and come mainly from the linear power spectrum at $k<0.09 h / \mathrm{Mpc}$. More sophisticated treatments of galaxy bias in which $Q_{\mathrm{nl}}$ is effectively computed from theoretical models constrained by small-scale clustering may eventually obviate the need to marginalize over this nuisance parameter, increasing the leverage of our measurements for constraining the linear power spectrum shape [99].

\section{E. Robustness to systematic errors}

Let us now consider potential systematic errors in the LRG data that could affect our results. Examples of such effects include radial modulations (due to misestimates of the radial selection function) and angular modulations (due to effects such as uncorrected dust extinction, variable observing conditions, photometric calibration errors and fiber collisions) of the density field. As long as such effects are uncorrelated with the cosmic density field, they will tend to add rather than subtract power.

\section{Analysis of subsets of galaxies}

To test for effects that would be expected to vary across the sky (depending on, say, reddening, seasonally variable photometric calibration errors, or observing conditions such as seeing and sky brightness), we repeat our entire analysis for the seven different angular subsets of the sky shown in Fig. 3 in search of inconsistencies. To search for potential zero-point offsets and other systematic effects associated with the southern Galactic stripes, they are defined as one of these seven angular subsets (see Fig. 3). To test for effects that depend on redshift, we use the measurements for our three redshift slices, plotted in Fig. 6.

To test the null hypothesis that all these subsamples are consistent with having the same power spectrum, we fit them all to our WMAP + LRG best-fit vanilla model described in Section IV, including our nonlinear correction (this $P(k)$ curve is quite similar to the best-fit WMAP3 model plotted above in, e.g., Fig. 4). We include the 20 band-powers with $k \lesssim 0.2$ in our fit, so if the null hypothesis is correct, we expect a mean $\chi^{2}$ of 20 with a standard deviation of $\sqrt{2 \times 20} \approx 6.3$. Our seven angular subsamples give a mean $\left\langle\chi^{2}\right\rangle \approx 22.6$ and a scatter $\left\langle\left(\chi^{2}-\right.\right.$ $\left.20)^{2}\right\rangle^{1 / 2} \approx 6$.9. Our three radial subsamples give $\left\langle\chi^{2}\right\rangle \approx$ 18.6 and $\left\langle\left(\chi^{2}-20\right)^{2}\right\rangle^{1 / 2} \approx 2.4$. All of the ten $\chi^{2}$-values are statistically consistent with the null hypothesis at the 95\% level. We also repeated the cosmological parameter analysis reported below with the southern stripes omitted, finding no significant change in the measured parameter values. In other words, all our angular and radial subsamples are consistent with having the same power spectrum, so these tests reveal no evidence for systematic errors causing radial or angular power spectrum variations.

\section{Analysis of subsets of modes}

Because of their angular or radial nature, all potential systematic errors discussed above create excess power mainly in the radial and angular modes. As mentioned 
above, one of the advantages of the PKL method is that it allows these modes to be excluded from the analysis, in analogy to the way potentially contaminated pixels in a CMB map can be excluded from a CMB power spectrum analysis. To quantify any such excess, we therefore repeat our full-sample analysis with radial and/or angular modes deleted. The results of this test are shown in Fig. 11 and are very encouraging; the differences are tiny. Any systematic errors adding power to these special modes would cause the black circles to lie systematically above the other points, but no such trend is seen, so there is no indication of excess radial or angular power in the data.

The slight shifts seen in the power on the largest scales are expected, since a non-negligible fraction of the information has been discarded on those scales. Figure 11 shows that removing the special modes results in a noticeable error bar increase on the largest scales and essentially no change on smaller scales. This can be readily understood geometrically. If we count the number of modes that probe mainly scales $k<k_{*}$, then the number of purely radial, purely angular and arbitrary modes will grow as $k_{*}, k_{*}^{2}$, and $k_{*}^{3}$, respectively. This means that "special"

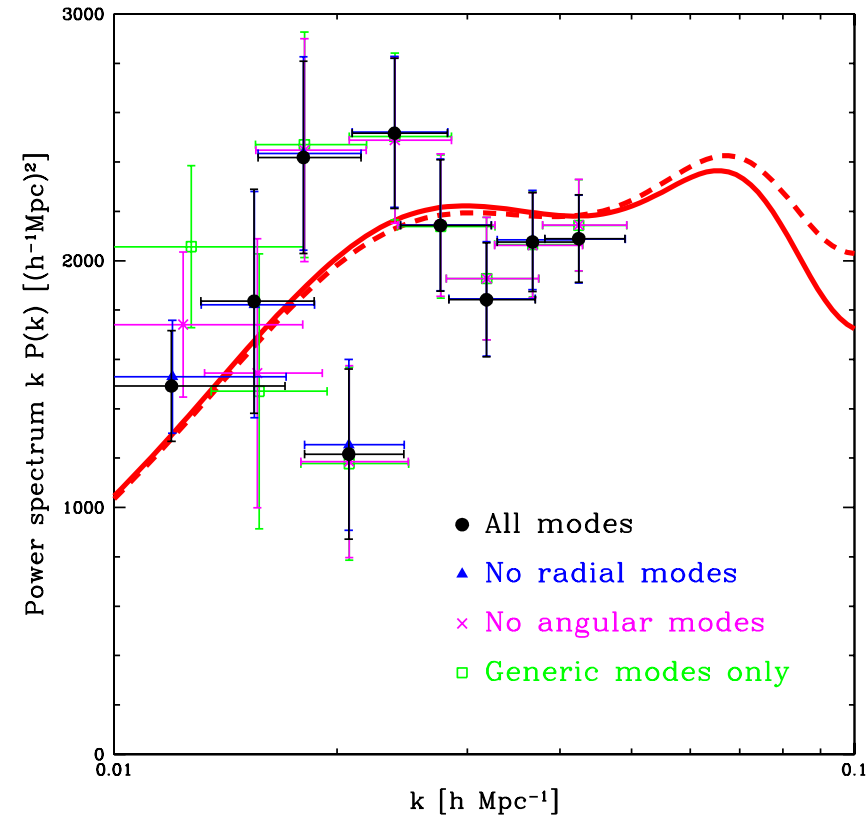

FIG. 11 (color online). Same as Fig. 7, but showing the effect of discarding special modes on the large-scale power. The circles with associated error bars correspond to our measured power spectrum using all 4000 full-sample PKL modes. The other points show the effect of removing the 332 purely angular modes (crosses), the 18 purely radial modes (triangles), and all special modes combined (squares), including seven associated with the motion of the local group as described in [28]. Any systematic errors adding power to these special modes would cause the black circles to lie systematically above the other points. These special modes are seen to have less impact at larger $k$ because they are outnumbered: the number of radial, angular, and generic modes below a given $k$-value scales as $k, k^{2}$, and $k^{3}$, respectively. modes (radial and angular) will make up a larger fraction of the total pool on large scales (at small $k$ ), and that the purely radial ones will be outnumbered by the purely angular ones. Conversely, the first 12 modes are all special ones: the monopole, the seven modes related to local-group motion, one radial mode and three angular modes. This means that almost all information on the very largest scales is lost when discarding special modes. Figure 11 illustrates this with the leftmost point labeled "generic" both having large error bars and being shifted to the right, where more information remains - yet it is consistent, lying about $1.3 \sigma$ above an imaginary line between the two leftmost black points. We also repeated the cosmological parameter analysis reported below with the special modes omitted, finding no significant change in the measured parameter values.

\section{F. Other tests}

We have found no evidence for systematic errors afflicting our power spectrum, suggesting that such effects, if present, are substantially smaller than our statistical errors. For additional bounds on potential systematic errors in the SDSS LRG sample, see [43].

A direct comparison of our $P(k)$-measurement and that of [43] is complicated because these are not measurements of the same function. [43] measures the angle-averaged redshift-space galaxy power spectrum, whereas our PKLmethod attempts to recover the real-space galaxy power spectrum, using finger-of-god (FOG) compression and linear theory to remove redshift-space distortion effects [28]. The galaxy selection is also different, with [43] mixing main-sample galaxies in with the LRGs. Both of these differences are expected to affect the nonlinear corrections. In addition, the quantity $P(k)$ plotted in [43] has correlated points with broader window functions than our uncorrelated points, and the angular coverage of the sample used in [43] is about 20\% larger. To make a direct but approximate comparison with [43], we perform our own FKP analysis, both with and without FOG-compression, and as described in Appendix A 1, we obtain good agreement with [43] on linear scales for the case of no defogging.

We further investigate the robustness of our results to systematic errors in Sec. IV F below, this time focusing on their potential impact on cosmological parameters.

\section{COSMOLOGICAL PARAMETERS}

Let us now explore the cosmological implications of our measurements by combining them with those from WMAP. As there has recently been extensive work on constraining cosmological parameters by combining multiple cosmological data sets involving CMB, galaxy clustering, Lyman $\alpha$ Forest, gravitational lensing, supernovae Ia and other probes (see, in particular, [7,39]), we will focus more narrowly on what can be learned from 
WMAP and the LRGs alone. This is interesting for two reasons:

(1) Less is more, in the sense that our results hinge on fewer assumptions about data quality and modeling. The WMAP and LRG power spectra suffice to break all major degeneracies within a broad class of models, yet they are also two remarkably clean measurements, probing gravitational clustering only on very large scales where complicated nonlinear physics is unlikely to cause problems.

(2) Since the LRG power spectrum is likely to be included (together with WMAP and other data sets) in future parameter analyses by other groups, it is important to elucidate what information it contains about cosmological parameters. We will therefore place particular emphasis on clarifying the links between cosmological parameters and observable features of both the LRG and WMAP power spectra, notably the LRG matter-radiation equality scale, the LRG acoustic scale, the CMB acoustic scale, unpolarized $\mathrm{CMB}$ peak height ratios and large-scale CMB polarization.

We then compare our constraints with those from other cosmological probes in Sec. VC. We also compare our results with the analysis of [36] below, which had the narrower focus of measuring the LRG acoustic scale; the correlation function analysis in that paper complements our present analysis, since the acoustic oscillations in $P(k)$ correspond to a readily measured single localized feature in real space $[36,102]$.

TABLE III. How key cosmological parameter constraints depend on data used and on assumptions about other parameters. The columns compare different theoretical priors indicated by numbers in italics. $w_{c}$ denotes dark energy that can cluster as in [7]. Rows labeled "+SDSS” combine WMAP3 and SDSS LRG data.

\begin{tabular}{|c|c|c|c|c|c|c|c|c|}
\hline & Data & Vanilla & Vanilla $+\Omega_{\text {tot }}$ & Vanilla $+r$ & Vanilla $+\alpha$ & Vanilla $+\omega_{\nu}$ & Vanilla $+w$ & Vanilla $+w_{c}$ \\
\hline \multirow[t]{2}{*}{$\Omega_{\text {tot }}$} & WMAP & 1 & $1.054_{-0.046}^{+0.064}$ & 1 & 1 & 1 & 1 & 1 \\
\hline & + SDSS & 1 & $\begin{array}{l}1.003_{-0.009}^{+0.010} \\
\end{array}$ & 1 & 1 & 1 & 1 & 1 \\
\hline \multirow[t]{2}{*}{$\Omega_{\Lambda}$} & WMAP & $0.761_{-0.037}^{+0.032}$ & $0.60_{-0.17}^{+0.14}$ & $0.805_{-0.042}^{+0.038}$ & $0.708_{-0.060}^{+0.051}$ & $0.651_{-0.086}^{+0.082}$ & $0.704_{-0.100}^{+0.071}$ & $0.879_{-0.168}^{+0.064}$ \\
\hline & + SDSS & $0.761_{-0.018}^{+0.017}$ & $0.757_{-0.021}^{+0.020}$ & $0.771_{-0.019}^{+0.018}$ & $0.750_{-0.022}^{+0.020}$ & $0.731_{-0.030}^{+0.024}$ & $0.757_{-0.020}^{+0.019}$ & $0.762_{-0.021}^{+0.020}$ \\
\hline \multirow[t]{2}{*}{$\Omega_{m}$} & WMAP & $0.239_{-0.032}^{+0.037}$ & $0.46_{-0.19}^{+0.23}$ & $0.195_{-0.038}^{+0.042}$ & $0.292_{-0.051}^{+0.060}$ & $0.349_{-0.082}^{+0.086}$ & $0.30_{-0.07}^{+0.10}$ & $0.12_{-0.06}^{+0.17}$ \\
\hline & $+\mathrm{SDSS}$ & $0.239_{-0.017}^{+0.018}$ & $0.246_{-0.025}^{+0.028}$ & $0.229_{-0.018}^{+0.019}$ & $0.250_{-0.020}^{+0.022}$ & $0.269_{-0.024}^{+0.030}$ & $0.243_{-0.019}^{+0.020}$ & $0.238_{-0.020}^{+0.021}$ \\
\hline \multirow[t]{2}{*}{$\omega_{m}$} & WMAP & $0.1272_{-0.0080}^{+0.0082}$ & $0.1277_{-0.0079}^{+0.0082}$ & $0.1194_{-0.0092}^{+0.0096}$ & $0.135_{-0.009}^{+0.010}$ & $0.139_{-0.011}^{+0.011}$ & $0.1274_{-0.0082}^{+0.0083}$ & $0.1269_{-0.0080}^{+0.0082}$ \\
\hline & $+\mathrm{SDSS}$ & $0.1272_{-0.0043}^{+0.0044}$ & $0.1260_{-0.0064}^{+0.0066}$ & $0.1268_{-0.0042}^{+0.0043}$ & $0.1271_{-0.0044}^{+0.0045}$ & $0.1301_{-0.0044}^{+0.0048}$ & $0.1248_{-0.0059}^{+0.0063}$ & $0.1264_{-0.0079}^{+0.0075}$ \\
\hline \multirow[t]{2}{*}{$\omega_{b}$} & WMAP & $0.0222_{-0.0007}^{+0.0007}$ & $\begin{array}{l}0.0218_{-0.0008}^{+0.0008} \\
n^{2}\end{array}$ & $0.0233_{-0.0010}^{+0.0011}$ & $0.0210_{-0.0010}^{+0.0010}$ & $0.0215_{-0.0009}^{+0.0009}$ & $0.0221_{-0.0007}^{+0.0007}$ & $0.0222_{-0.0007}^{+0.0008}$ \\
\hline & + SDSS & $0.0222_{-0.0007}^{+0.0007}$ & $0.0222_{-0.0007}^{+0.0007}$ & $0.0229_{-0.0008}^{+0.0009}$ & $0.0213_{-0.0010}^{+0.0010}$ & $0.0221_{-0.0008}^{+0.0008}$ & $0.0223_{-0.0007}^{+0.0007}$ & $0.0224_{-0.0007}^{+0.0008}$ \\
\hline \multirow[t]{2}{*}{$\omega_{\nu}$} & WMAP & 0 & 0 & 0 & 0 & $<0.024(95 \%)$ & 0 & 0 \\
\hline & $+\mathrm{SDSS}$ & 0 & 0 & 0 & 0 & $<0.010(95 \%)$ & 0 & 0 \\
\hline \multirow[t]{2}{*}{$M_{\nu}$} & WMAP & 0 & 0 & 0 & 0 & $<2.2(95 \%)$ & 0 & 0 \\
\hline & + SDSS & 0 & 0 & 0 & 0 & $<0.94(95 \%)$ & 0 & 0 \\
\hline \multirow[t]{2}{*}{$w$} & WMAP & -1 & -1 & -1 & -1 & -1 & $-0.82_{-0.19}^{+0.23}$ & $-1.69_{-0.85}^{+0.88}$ \\
\hline & $+\mathrm{SDSS}$ & -1 & -1 & -1 & -1 & -1 & $-0.941_{-0.101}^{+0.087}$ & $-1.00_{-0.19}^{+0.17}$ \\
\hline \multirow[t]{2}{*}{$\sigma_{8}$} & WMAP & $0.758_{-0.051}^{+0.050}$ & $0.732_{-0.046}^{+0.051}$ & $0.706_{-0.072}^{+0.064}$ & $0.776_{-0.053}^{+0.056}$ & $0.597_{-0.075}^{+0.085}$ & $0.736_{-0.052}^{+0.054}$ & $0.747_{-0.066}^{+0.1966}$ \\
\hline & + SDSS & $0.756_{-0.035}^{+0.035}$ & $0.747_{-0.044}^{+0.046}$ & $0.751_{-0.036}^{+0.036}$ & $0.739_{-0.035}^{+0.036}$ & $0.673_{-0.061}^{+0.056}$ & $0.733_{-0.043}^{+0.048}$ & $0.745_{-0.056}^{+0.057}$ \\
\hline \multirow[t]{2}{*}{$r_{.002}$} & WMAP & 0 & 0 & $<0.65(95 \%)$ & 0 & 0 & 0 & 0 \\
\hline & + SDSS & 0 & 0 & $<0.33(95 \%)$ & 0 & 0 & 0 & 0 \\
\hline \multirow[t]{2}{*}{$n_{s}$} & WMAP & $0.954_{-0.016}^{+0.017}$ & $0.943_{-0.016}^{+0.017}$ & $0.982_{-0.026}^{+0.032}$ & $0.871_{-0.046}^{+0.047}$ & $0.928_{-0.024}^{+0.022}$ & $0.945_{-0.016}^{+0.017}$ & $0.947_{-0.017}^{+0.019}$ \\
\hline & + SDSS & $0.953_{-0.016}^{+0.016}$ & $0.952_{-0.016}^{+0.011}$ & $0.967_{-0.020}^{+0.022}$ & $0.895_{-0.042}^{+0.041}$ & $0.945_{-0.017}^{+0.024}$ & $0.950_{-0.016}^{+0.016}$ & $0.953_{-0.017}^{+0.018}$ \\
\hline \multirow[t]{2}{*}{$\alpha$} & WMAP & 0 & 0 & 0 & $-0.056_{-0.031}^{+0.031}$ & 0 & 0 & 0 \\
\hline & + SDSS & 0 & 0 & 0 & $-0.040_{-0.027}^{+0.027}$ & 0 & 0 & 0 \\
\hline \multirow[t]{2}{*}{$h$} & WMAP & $0.730_{-0.031}^{+0.033}$ & $0.53_{-0.10}^{+0.15}$ & $0.782_{-0.047}^{+0.058}$ & $0.679_{-0.040}^{+0.044}$ & $0.630_{-0.044}^{+0.065}$ & $0.657_{-0.086}^{+0.085}$ & $1.03_{-0.37}^{+0.46}$ \\
\hline & $+\mathrm{SDSS}$ & $0.730_{-0.019}^{+0.019}$ & $0.716_{-0.043}^{+0.047}$ & $0.744_{-0.021}^{+0.022}$ & $0.713_{-0.022}^{+0.022}$ & $0.695_{-0.028}^{+0.025}$ & $0.716_{-0.029}^{+0.031}$ & $0.727_{-0.034}^{+0.037}$ \\
\hline \multirow[t]{2}{*}{$t_{\text {now }}$} & WMAP & $13.75_{-0.16}^{+0.16}$ & $16.0_{-1.8}^{+1.5}$ & $13.53_{-0.25}^{+0.21}$ & $13.98_{-0.20}^{+0.20}$ & $14.31_{-0.33}^{+0.24}$ & $13.96_{-0.28}^{+0.34}$ & $13.44_{-0.27}^{+0.49}$ \\
\hline & $+\mathrm{SDSS}$ & $13.76_{-0.15}^{+0.15}$ & $13.93_{-0.58}^{+0.59}$ & $13.65_{-0.18}^{+0.17}$ & $13.90_{-0.19}^{+0.19}$ & $13.98_{-0.20}^{+0.22}$ & $13.80_{-0.17}^{+0.18}$ & $13.77_{-0.24}^{+0.26}$ \\
\hline \multirow[t]{2}{*}{$\tau$} & WMAP & $0.090_{-0.029}^{+0.029}$ & $0.083_{-0.029}^{+0.029}$ & $0.091_{-0.032}^{+0.031}$ & $0.101_{-0.031}^{+0.031}$ & $0.082_{-0.030}^{+0.029}$ & $0.087_{-0.031}^{+0.030}$ & $0.087_{-0.030}^{+0.030}$ \\
\hline & + SDSS & $0.087_{-0.030}^{+0.028}$ & $0.088_{-0.031}^{+0.029}$ & $0.085_{-0.031}^{+0.029}$ & $0.101_{-0.032}^{+0.032}$ & $0.087_{-0.029}^{+0.028}$ & $0.090_{-0.031}^{+0.030}$ & $0.089_{-0.032}^{+0.030}$ \\
\hline \multirow[t]{2}{*}{$b$} & WMAP & & & & & & & \\
\hline & $+\mathrm{SDSS}$ & $1.896_{-0.069}^{+0.074}$ & $1.911_{-0.086}^{+0.092}$ & $1.919_{-0.072}^{+0.078}$ & $1.853_{-0.077}^{+0.081}$ & $2.03_{-0.10}^{+0.11}$ & $1.897_{-0.072}^{+0.076}$ & $1.92_{-0.08}^{+0.10}$ \\
\hline \multirow[t]{2}{*}{$Q_{\mathrm{nl}}$} & WMAP & & & & & & & \\
\hline & $+\mathrm{SDSS}$ & $30.3_{-4.1}^{+4.4}$ & $30.0_{-4.2}^{+4.6}$ & $30.9_{-4.1}^{+4.5}$ & $34.7_{-5.4}^{+6.1}$ & $34.9_{-5.3}^{+6.9}$ & $31.0_{-4.3}^{+4.7}$ & $31.0_{-4.4}^{+5.0}$ \\
\hline \multirow[t]{2}{*}{$\Delta \chi^{2}$} & WMAP & 0.0 & -2.0 & 0.0 & -3.6 & -1.0 & -1.0 & 0.0 \\
\hline & $+\mathrm{SDSS}$ & 0.0 & 0.0 & -0.5 & -2.4 & -0.5 & -0.9 & -0.3 \\
\hline
\end{tabular}


We work within the context of the arguably simplest inflationary scenario that fits our data. This is a hot big bang cosmology with primordial fluctuations that are adiabatic (i.e., we do not include isocurvature modes) and Gaussian, with negligible generation of fluctuations by cosmic strings, textures or domain walls. We assume the standard model of particle physics with three active neutrino species, very slightly heated during the era of electron/positron annihilation [103]. Within this framework, we parameterize our cosmological model in terms of 12
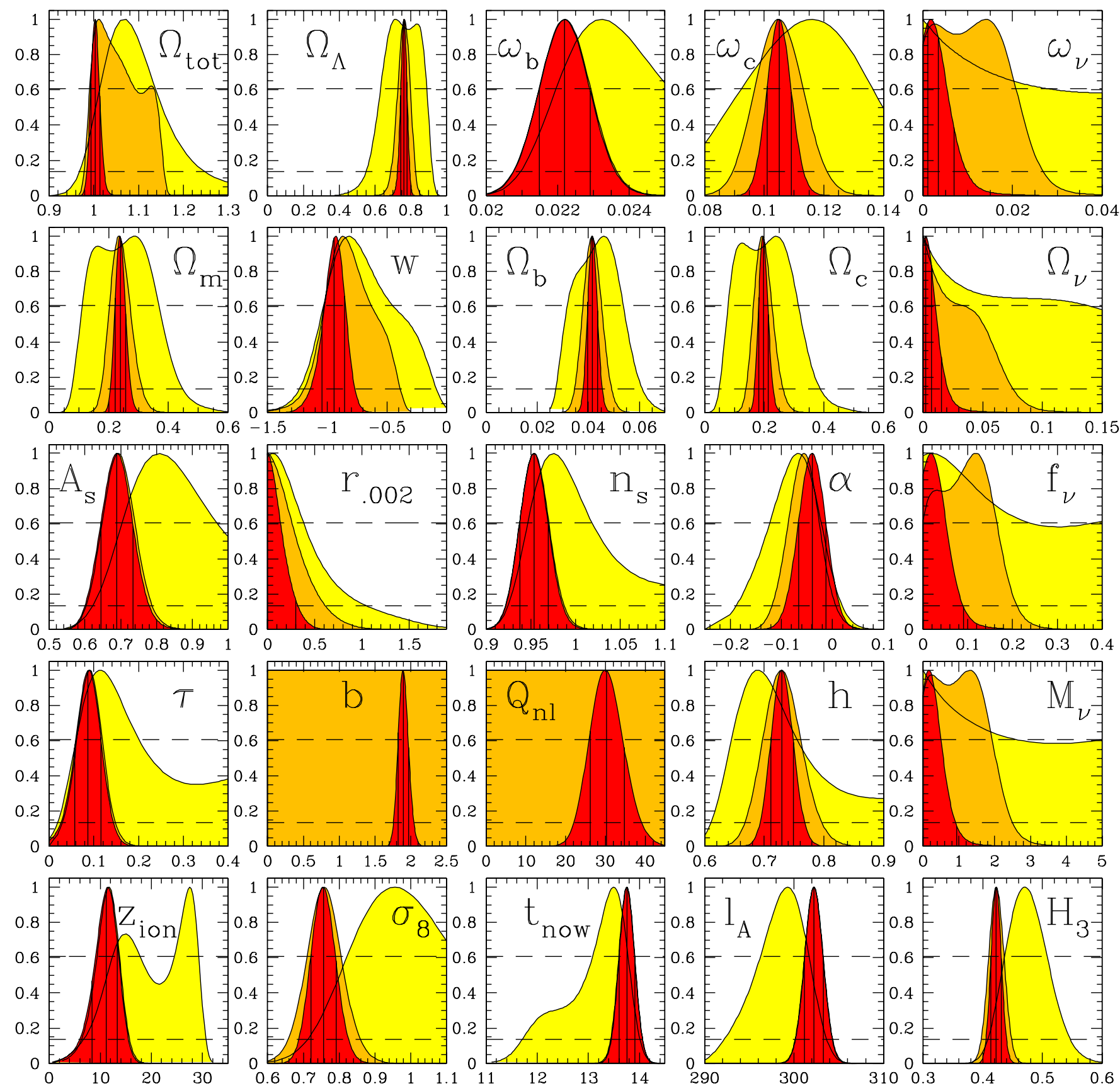

FIG. 12 (color online). Constraints on key individual cosmological quantities using WMAP1 (yellow/light gray distributions), WMAP3 (narrower orange/gray distributions) and including SDSS LRG information (red/dark gray distributions). If the orange/gray is completely hidden behind the red/dark gray, the LRGs thus add no information. Each distribution shown has been marginalized over all other quantities in the vanilla class of models parametrized by $\left(\Omega_{\Lambda}, \omega_{b}, \omega_{c}, A_{s}, n_{s}, \tau, b, Q_{\mathrm{nl}}\right)$. The parameter measurements and error bars quoted in the tables correspond to the median and the central $68 \%$ of the distributions, indicated by three vertical lines for the WMAP3 + SDSS case above. When the distribution peaks near zero (like for $r$ ), we instead quote an upper limit at the 95th percentile (single short vertical line). The horizontal dashed lines indicate $e^{-x^{2} / 2}$ for $x=1$ and 2 , respectively, so if the distribution were Gaussian, its intersections with these lines would correspond to $1 \sigma$ and $2 \sigma$ limits, respectively. 
parameters that are nowadays rather standard, augmented with the two nuisance parameters $b$ and $Q_{\mathrm{nl}}$ from Eq. (4):

$$
\mathbf{p} \equiv\left(\Omega_{\mathrm{tot}}, \Omega_{\Lambda}, \omega_{b}, \omega_{\mathrm{c}}, \omega_{\nu}, w, A_{s}, r, n_{s}, n_{t}, \alpha, \tau, b, Q_{\mathrm{nl}}\right) .
$$

Table II defines these 14 parameters and another 45 that can be derived from them; in essence, $\left(\Omega_{\text {tot }}, \Omega_{\Lambda}, \omega_{b}, \omega_{c}\right.$, $\left.\omega_{\nu}, w\right)$ define the cosmic matter budget, $\left(A_{s}, n_{s}, \alpha, r, n_{t}\right)$ specify the seed fluctuations and $\left(\tau, b, Q_{\mathrm{nl}}\right)$ are nuisance parameters. We will frequently use the term "vanilla" to refer to the minimal model space parametrized by $\left(\Omega_{\Lambda}, \omega_{b}, \omega_{\mathrm{c}}, A_{s}, n_{s}, \tau, b, Q_{\mathrm{nl}}\right), \quad$ setting $\omega_{\nu}=\alpha=r=$ $n_{t}=0, \Omega_{\text {tot }}=1$, and $w=-1$; this is the smallest subset of our parameters that provides a good fit to our data. Since current $n_{t}$-constraints are too weak to be interesting, we make the slow-roll assumption $n_{t}=-r / 8$ throughout this paper rather than treat $n_{t}$ as a free parameter.

All our parameter constraints were computed using the now standard Monte Carlo Markov chain (MCMC) approach [104-110] as implemented in [33]. ${ }^{2}$

\section{A. Basic results}

Our constraints on individual cosmological parameters are given in Tables II and III and illustrated in Fig. 12, both for WMAP alone and when including our SDSS LRG information. Table II and Fig. 12 take the Occam's razor approach of marginalizing only over vanilla parameters $\left(\Omega_{\Lambda}, \omega_{b}, \omega_{c}, A_{s}, n_{s}, \tau, b, Q_{\mathrm{nl}}\right)$, whereas Table III shows how key results depend on assumptions about the nonvanilla parameters $\left(\Omega_{\mathrm{tot}}, \omega_{\nu}, w, r, \alpha\right)$ introduced one at a time. In other words, Table II and Fig. 12 use the vanilla assumptions by default; for example, models with $\omega_{\nu} \neq 0$ are used only for the constraints on $\omega_{\nu}$ and other neutrino parameters $\left(\Omega_{\nu}, \xi_{\nu}, f_{\nu}\right.$, and $\left.M_{\nu}\right)$.

The parameter measurements and error bars quoted in the tables correspond to the median and the central $68 \%$ of the probability distributions, indicated by three vertical lines in Fig. 12. When a distribution peaks near zero, we

\footnotetext{
${ }^{2}$ To mitigate numerically deleterious degeneracies, the independent MCMC variables are chosen to be the parameters $\left(\Theta_{s}, \Omega_{\Lambda}, \omega_{b}, \omega_{d}, f_{\nu}, w, A_{\text {peak }}, n_{s}, \alpha, r, n_{t}, A_{\tau}, b, Q_{\mathrm{nl}}\right) \quad$ from Table II, where $\omega_{d} \equiv \omega_{\mathrm{c}}+\omega_{\nu}$, i.e., $\left(\Omega_{\mathrm{tot}}, \omega_{\mathrm{c}}, \omega_{\nu}, A_{s}, \tau\right)$ are replaced by $\left(\Theta_{s}, \omega_{d}, f_{\nu}, A_{\text {peak }}, e^{-2 \tau}\right)$ as in $[33,111]$. When imposing a flatness prior $\Omega_{\text {tot }}=1$, we retained $\Theta_{s}$ as a free parameter and dropped $\Omega_{\Lambda}$. The WMAP3 log-likelihoods are computed with the software provided by the WMAP team or taken from WMAP team chains on the LAMBDA archive (including all unpolarized and polarized information) and fit by a multivariate 4th order polynomial [112] for more rapid MCMC-runs involving galaxies. The SDSS likelihood uses the LRG sample alone and is computed with the software available at http://space.mit.edu/home/tegmark/sdss/ and described in Appendix A 4, employing only the measurements with $k \leq$ $0.2 h / \mathrm{Mpc}$ unless otherwise specified. Our WMAP3 + SDSS chains have $3 \times 10^{6}$ steps each and are thinned by a factor of 10. To be conservative, we do not use our SDSS measurement of the redshift-space distortion parameter $\beta$, nor do we use any other information ("priors") whatsoever unless explicitly stated.
}

instead quote an upper limit at the 95th percentile. Note that the tabulated median values are near but not identical to those of the maximum likelihood model. Our best-fit vanilla model has $\Omega_{\Lambda}=0.763, \omega_{b}=0.0223, \omega_{\mathrm{c}}=0.105$, $A_{s}=0.685, n_{s}=0.954, \tau=0.0842, b=1.90, Q_{\mathrm{nl}}=$ 31.0. As customary, the $2 \sigma$ contours in the numerous two-parameter figures below are drawn where the likelihood has dropped to 0.0455 of its maximum value, which corresponds to $\Delta \chi^{2} \approx 6.18$ and $95.45 \% \approx 95 \%$ enclosed probability for a two-dimensional Gaussian distribution.

We will spend most of the remainder of this paper digesting this information one step at a time, focusing on what WMAP and SDSS do and do not tell us about the underlying physics, and on how robust the constraints are to assumptions about physics and data sets. The onedimensional constraints in the tables and Fig. 12 fail to reveal important information hidden in parameter correlations and degeneracies, so we will study the joint constraints on key 2-parameter pairs. We will begin with the vanilla 6-parameter space of models, then introduce additional parameters (starting in Sec. IV B) to quantify both how accurately we can measure them and to what extent they weaken the constraints on the other parameters.

First, however, some of the parameters in Table II deserve comment. The additional parameters below the double line in Table II are all determined by those above the double line by simple functional relationships, and fall into several groups.

Together with the usual suspects under the heading "other popular parameters," we have included alternative fluctuation amplitude parameters: to facilitate comparison with other work, we quote the seed fluctuation amplitudes not only at the scale $k=0.05 / \mathrm{Mpc}$ employed by CMBfast [113], CAMB [114], and CosmoMC [108] (denoted $A_{s}$ and $r$ ), but also at the scale $k=0.002 / \mathrm{Mpc}$ employed by the WMAP team in [7] (denoted $A_{.002}$ and $r_{.002}$ ).

The "cosmic history parameters" specify when our universe became matter-dominated, recombined, reionized, started accelerating ( $\ddot{a}>0$ ), and produced us.

Those labeled "fundamental parameters" are intrinsic properties of our universe that are independent of our observing epoch $t_{\text {now }}$. (In contrast, most other parameters would have different numerical values if we were to measure them, say, $10 \mathrm{Gyr}$ from now. For example, $t_{\text {now }}$ would be about $24 \mathrm{Gyr}, z_{\mathrm{eq}}$ and $\Omega_{\Lambda}$ would be larger, and $h, \Omega_{m}$ and $\omega_{\mathrm{m}}$ would all be smaller. Such parameters are therefore not properties of our universe, but merely alternative time variables.)

The $Q$-parameter (not to be confused with $Q_{\mathrm{nl}}$ ) is the primordial density fluctuation amplitude $\sim 10^{-5}$. The curvature parameter $\kappa$ is the curvature that the Universe would have had at the Planck time if there was no inflationary epoch, and its small numerical value $\sim 10^{-61}$ constitutes the flatness problem that inflation solves. $\left(\xi, \xi_{b}, \xi_{c}, \xi_{\nu}\right)$ are the fundamental parameters corresponding to the cosmologi- 
cally popular quartet $\left(\Omega_{m}, \Omega_{b}, \Omega_{c}, \Omega_{\nu}\right)$, giving the densities per CMB photon. The current densities are $\rho_{i}=\rho_{h} \omega_{i}$, where $i=m, b, c, \nu$, and $\rho_{h}$ denotes the constant reference density $3(H / h)^{2} / 8 \pi G=3\left(100 \mathrm{~km} \mathrm{~s}^{-1} \mathrm{Mpc}^{-1}\right)^{2} / 8 \pi G \approx$ $1.87882 \times 10^{-26} \mathrm{~kg} / \mathrm{m}^{3}$, so the conversion between the conventional and fundamental density parameters is $\xi_{i} \equiv$ $\rho_{i} / n_{\gamma} \approx 25.646 \mathrm{eV} \times\left(T_{\mathrm{cmb}} / 2.726 \mathrm{~K}\right) \omega_{i}$ in units where $c=1$. The parameter $\xi_{m}$ is of the same order as the temperature at matter-radiation equality temperature, $k T_{\mathrm{eq}} \approx 0.22 \xi[115] .^{3}$

The tiny value $\sim 10^{-123}$ of the vacuum density $\rho_{\Lambda}$ in Planck units where $c=G=\hbar=1$ constitutes the wellknown cosmological constant problem, and the tiny yet similar value of the parameter combination $Q^{3} \xi^{4}$ explains the origin of attempts to explain this value anthropically [116-123]: $Q^{3} \xi^{4}$ is roughly the density of the universe at the time when the first nonlinear dark matter halos would form if $\rho_{\Lambda}=0$ [115], so if $\rho_{\Lambda} \gg Q^{3} \xi^{4}$, dark energy freezes fluctuation growth before then and no nonlinear structures ever form.

The parameters $\left(A_{\Lambda}, \sigma_{\mathrm{gal}}^{*}\right)$ are useful for anthropic buffs, since they directly determine the density fluctuation history on galaxy scales through equation (5) in [95] (where $\sigma_{\text {gal }}^{*}$ is denoted $\left.\sigma_{M}(0)\right)$. Roughly, fluctuations grow from the initial level $\sigma_{\text {gal }}^{*}$ by a factor $A_{\Lambda}$. Marginalizing over the neutrino fraction gives $A_{\Lambda}=2279_{-182}^{+240}, \quad \sigma_{\text {gal }}^{*}=$ $0.538_{-0.022}^{+0.024} \times 10^{-3}$.

The group labeled "CMB phenomenology parameters" contains parameters that correspond rather closely to the quantities most accurately measured by the CMB, such as heights and locations of power spectrum peaks. Many are seen to be measured at the percent level or better. These parameters are useful for both numerical and intuitionbuilding purposes $[96,111,112,124-126]$. Whereas CMB constraints suffer from severe degeneracies involving physical parameters further up in the table (involving, e.g., $\Omega_{\text {tot }}$, and $\Omega_{\Lambda}$ as discussed below), these phenomenological parameters are all constrained with small and fairly uncorrelated measurement errors. By transforming the multidimensional WMAP3 log-likelihood function into the space spanned by $\left(H_{2}, \omega_{\mathrm{m}}, f_{\nu}, \Omega_{\Lambda}, w, \Theta_{s}, A_{\text {pivot }}, H_{3}, \alpha\right.$, $\left.r, n_{t}, A_{\tau}, b, Q_{\mathrm{nl}}\right)$, it becomes better approximated by our quartic polynomial fit described in Footnote 2 and [112]: for example, the rms error is a negligible $\Delta \ln \mathcal{L} \approx 0.03$ for the vanilla case. Roughly speaking, this transformation replaces the curvature parameter $\Omega_{\text {tot }}$ by the characteristic peak scale $\Theta_{s}$, the baryon fraction by the ratio $\mathrm{H}_{2}$ of the

${ }^{3}$ The matter-radiation equality temperature is given by

$$
k T_{\text {eq }}=\frac{30 \zeta(3)}{\pi^{4}}\left[1+\frac{7}{8} N_{\nu}\left(\frac{4}{11}\right)^{4 / 3}\right]^{-1} \xi \approx 0.2195 \xi,
$$

where $\zeta(3) \approx 1.202$, and the effective number of neutrino species in the standard model is $N_{\nu} \approx 3.022$ [103] when taking into account the effect of electron-positron annihilation on the relic neutrino energy density. first two peak heights, the spectral index $n_{s}$ by the ratio $H_{3}$ of the third to first peak heights, and the overall peak amplitude $A_{\text {peak }}$ by the amplitude $A_{\text {pivot }}$ at the pivot scale where it is uncorrelated with $n_{s}$. Aside from this numerical utility, these parameters also help demystify the "black box" aspect of CMB parameter constraints, elucidating their origin in terms of features in the data and in the physics [96].

\section{B. Vanilla parameters}

Figure 12 compares the constraints on key parameters from the 1-year WMAP data ("WMAP1"), the 3-year WMAP data ("WMAP3") and WMAP3 combined with our SDSS LRG measurements ("WMAP + LRG"). We include the WMAP1 case because it constitutes a welltested baseline and illustrates both the dramatic progress in the field and what the key new WMAP3 information is, particularly from $E$-polarization.

\section{What WMAP3 adds}

The first thing to note is the dramatic improvement from WMAP1 to WMAP3 emphasized in [7]. (Plotted WMAP1 constraints are from [33].) As shown in [127], this stems almost entirely from the new measurement of the low- $\ell E$ power spectrum, which detects the reionization signature at about $3 \sigma$ and determines the corresponding optical depth $\tau=0.09 \pm 0.03$. This measurement breaks the severe vanilla degeneracy in the WMAP1 data [32,33] (see Fig. 13) and causes the dramatic tightening of the constraints on $\left(\omega_{b}, \omega_{c}, \Omega_{\Lambda}, A_{s}, n_{s}\right)$ seen in the figures; essentially, with $\tau$ well constrained, the ratio of large-scale power to the acoustic peaks determines $n_{s}$, and the relative heights of the acoustic peaks then determine $\omega_{b}$ and $\omega_{\mathrm{c}}$ without residual uncertainty due to $n_{s}$. Indeed, [127] has shown that discarding all the WMAP3 polarization data (both TE and EE) and replacing it with a Gaussian prior $\tau=0.09 \pm$ 0.03 recovers parameter constraints essentially identical to those from the full WMAP3 data set. In Section IV F 1, we will return to the issue of what happens if this $\tau$-measurement is compromised by polarized foreground contamination.

The second important change from WMAP1 to WMAP3 is that the central values of some parameters have shifted noticeably [7]. Improved modeling of noise correlations and polarized foregrounds have lowered the low- $\ell$ TE power and thus eliminated the WMAP1 evidence for $\tau \sim$ 0.17 . Since the fluctuation amplitude scales as $e^{\tau}$ times the CMB peak amplitude, this $\tau$ drop of 0.08 would push $\sigma_{8}$ down by about $8 \%$. In addition, better measurements around the 3rd peak and a change in analysis procedure (marginalizing over the SZ-contribution) have lowered $\omega_{\mathrm{m}}$ by about $13 \%$, causing fluctuation growth to start later $\left(z_{\text {eq }}\right.$ decreases) and end earlier ( $z_{\text {acc }}$ increases), reducing $\sigma_{8}$ by another $8 \%$. These effects combine to lower $\sigma_{8}$ by about $21 \%$ when also taking into account the slight lowering of $n_{s}$. 


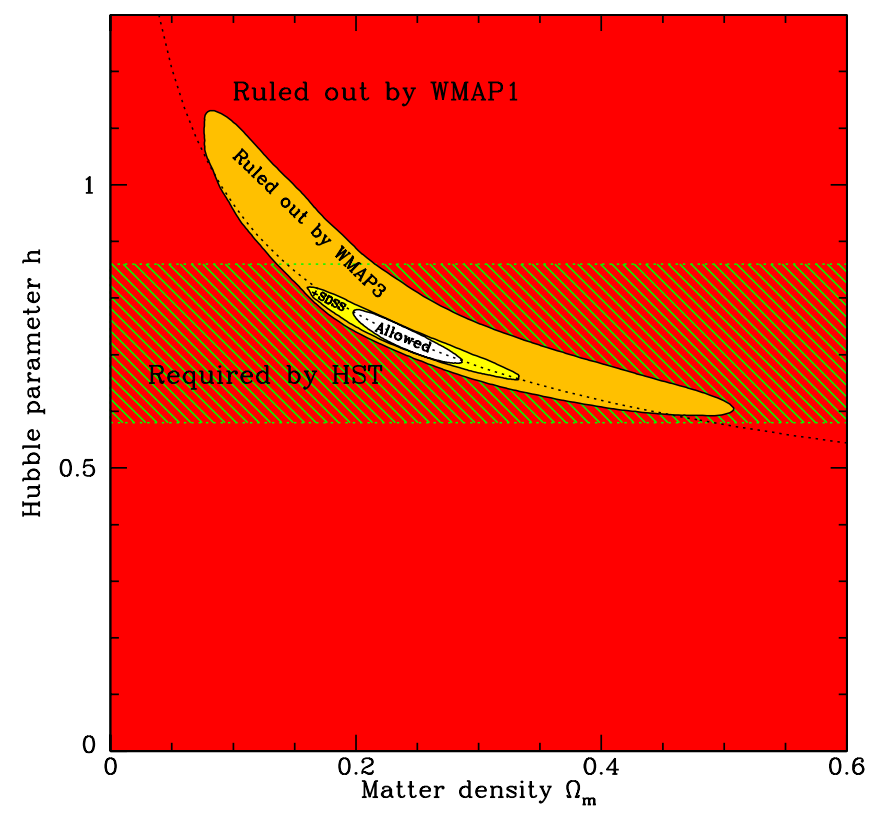

FIG. 13 (color online). $95 \%$ constraints in the $\left(\Omega_{m}, h\right)$ plane. For 6-parameter vanilla models, the shaded red/gray region is ruled out by WMAP1 and the shaded orange/gray region by WMAP3; the main source of the dramatic improvement is the measurement of $E$-polarization breaking the degeneracy involving $\tau$. Adding SDSS LRG information further constrains the parameters to the white region marked "Allowed." The horizontal hatched band is required by the HST key project [136]. The dotted line shows the fit $h=0.72\left(\Omega_{m} / 0.25\right)^{-0.32}$, explaining the origin of the percent-level constraint $h\left(\Omega_{m} / 0.25\right)^{0.32}=$ $0.719 \pm 0.008(1 \sigma)$.

\section{What SDSS LRGs add}

A key reason that non-CMB datasets such as the 2dFGRS and the SDSS improved WMAP1 constraints so dramatically was that they helped break the vanilla banana degeneracy seen in Fig. 13, so the fact that WMAP3 now mitigates this internally with its $E$-polarization measurement of $\tau$ clearly reduces the value added by other datasets. However, Table III shows that our LRG measurements nonetheless give substantial improvements, cutting error bars on $\Omega_{m}, \omega_{\mathrm{m}}$, and $h$ by about a factor of 2 for vanilla models and by up to almost an order of magnitude when curvature, tensors, neutrinos or $w$ are allowed.

The physics underlying these improvements is illustrated in Fig. 14. The cosmological information in the CMB splits naturally into two parts, one "vertical" and one "horizontal," corresponding to the vertical and horizontal positions of the power spectrum peaks.

By vertical information, we mean the relative heights of the acoustic peaks, which depend only on the physical matter densities $\left(\omega_{\mathrm{m}}, \omega_{b}, \omega_{\nu}\right)$ and the scalar primordial power spectrum shape $\left(n_{s}, \alpha\right)$. They are independent of curvature and dark energy, since $\Omega_{\Lambda}(z) \approx \Omega_{k}(z) \approx 0$ at $z \gtrsim 10^{3}$. They are independent of $h$, since the physics at those early times depended only on the expansion rate as a

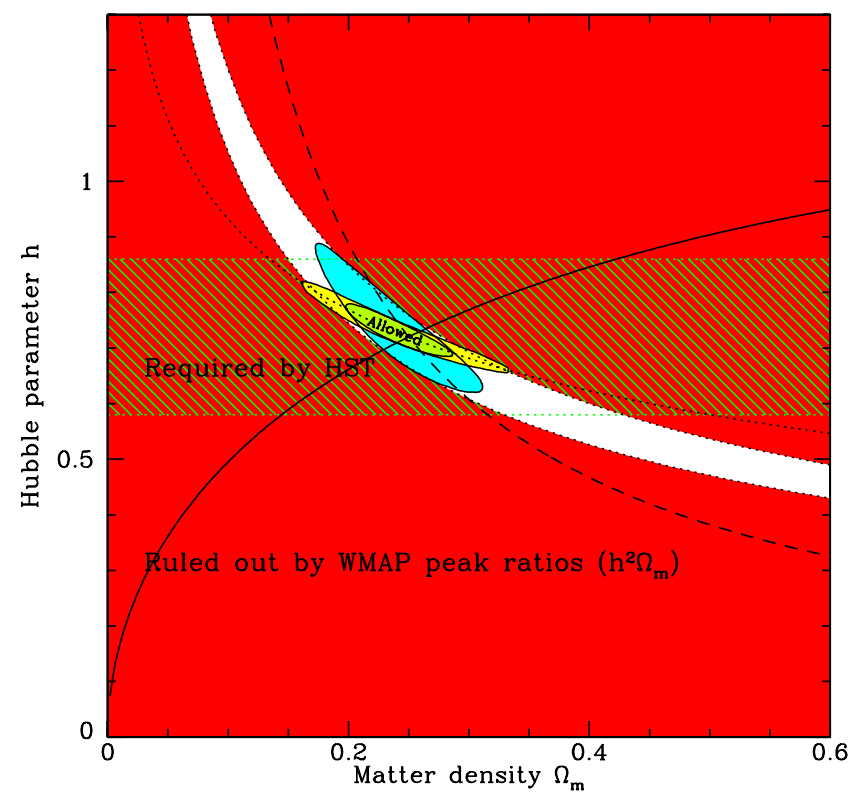

FIG. 14 (color online). Illustration of the physics underlying the previous figure. Using only WMAP CMB peak height ratios constrains $\left(\omega_{\mathrm{m}}, \omega_{b}, n_{s}\right)$ independently of $A_{s}, \tau$, curvature and late-time dark energy properties. This excludes all but the white band $\omega_{\mathrm{m}} \equiv h^{2} \Omega_{m}=0.127 \pm 0.017(2 \sigma)$. If we assume $\Omega_{\mathrm{tot}}=$ 1 and vanilla dark energy, we can supplement this with independent "standard ruler" information from either WMAP CMB (thin yellow/light gray ellipse) giving $\Omega_{m}=0.239 \pm 0.034$ $(1 \sigma)$, or SDSS galaxies (thicker blue/gray ellipse) giving $\Omega_{m}=$ $0.239 \pm 0.027(1 \sigma)$. These two rulers are not only beautifully consistent, but also complementary, with the joint constraints (small ellipse marked allowed) being tighter than those from using either separately, giving $\Omega_{m}=0.238 \pm 0.017(1 \sigma)$. The plotted 2-dimensional constraints are all $2 \sigma$. The three black curves correspond to constant "horizontal" observables: constant angular scales for the acoustic peaks in the CMB power (dotted, $h_{\sim}^{\propto} \Omega_{m}^{-0.3}$ ), for the acoustic peaks in the galaxy power (solid, $h_{\sim}^{\propto} \Omega_{m}^{0.37}$ ) and for the turnover in the galaxy power spectrum (dashed, $h_{\sim}^{\propto} \Omega_{m}^{-0.93}$ ). This illustrates why the galaxy acoustic scale is even more helpful than that of the CMB for measuring $\Omega_{m}$ : although it is currently less accurately measured, its degeneracy direction is more perpendicular to the CMB peak ratio measurement of $h^{2} \Omega_{m}$.

function of temperature back then, which is simply $\xi^{1 / 2} T^{3 / 2}$ times a known numerical constant, where $\xi$ is given by $\omega_{\mathrm{m}}$ and the current CMB temperature (see Table 3 in [115]). They are also conveniently independent of $\tau$ and $r$, which change the power spectrum shape only at $\ell \ll 10^{2}$.

By horizontal (a.k.a. "standard ruler") information, we mean the acoustic angular scale $\ell_{A} \equiv \pi d_{A}\left(z_{\text {rec }}\right) / r_{\mathrm{s}}\left(z_{\text {rec }}\right)$ defined in Table II. The $\ell$-values of CMB power spectrum peaks and troughs are all equal to $\ell_{A}$ times constants depending on $\left(\omega_{\mathrm{m}}, \omega_{b}\right)$, so changing $\ell_{A}$ by some factor by altering $\left(\Omega_{k}, \Omega_{\Lambda}, w\right)$ simply shifts the CMB peaks horizontally by that factor and alters the late integrated Sachs Wolfe effect at $\ell \ll 10^{2}$. Although this single number $\ell_{A}$ is now measured to great precision $(\sim 0.3 \%)$, it 
depends on multiple parameters, and it is popular to break this degeneracy with assumptions rather than measurements. The sound horizon at recombination $r_{\mathrm{s}}\left(z_{\mathrm{rec}}\right)$ in the denominator depends only weakly on $\left(\omega_{\mathrm{m}}, \omega_{b}\right)$, which are well constrained from the vertical information, and Table II shows that it is now known to about $1 \%$. In contrast, the comoving angular diameter distance to recombination $d_{A}\left(z_{\text {rec }}\right)$ depends sensitively on both the spatial curvature $\Omega_{k}$ and the cosmic expansion history $H(z)$, which in turn depends on the history of the dark energy density:

$$
\begin{aligned}
\frac{H(z)}{H_{0}}= & {\left[X(z) \Omega_{\Lambda}+(1+z)^{2} \Omega_{k}+(1+z)^{3} \Omega_{m}\right.} \\
& \left.\left.+(1+z)^{4} \Omega_{r}\right)\right]^{1 / 2} .
\end{aligned}
$$

Here $X(z)$ is defined as the dark energy density relative to its present value [128], with vanilla dark energy (a cosmological constant) corresponding to $X(z)=1$. The most common (although physically unmotivated) parametrization of this function in the literature has been a simple power law $X(z)=(1+z)^{3(1+w)}$, although it has also been constrained with a variety of other parametric and nonparametric approaches (see [129] and references therein). The parameter $\Omega_{r}$ refers to the radiation contribution from photons and massless neutrinos, which is given by $h^{2} \Omega_{r} \approx$ $0.0000416\left(T_{\mathrm{cmb}} / 2.726 \mathrm{~K}\right)^{4}$ and makes a negligible contribution at low redshift.

Using the vertical WMAP information alone gives a tight constraint on $\omega_{\mathrm{m}} \equiv h^{2} \Omega_{m}$, corresponding to the white band in Fig. 14, independent of assumptions about curvature or dark energy. ${ }^{4}$ To this robust measurement, we can now add two independent pieces of information if we are willing to make the vanilla assumptions that curvature vanishes and dark energy is a cosmological constant: If we add the WMAP horizontal information, the allowed region shrinks to the thin ellipse hugging the $h_{\sim}^{\propto} \Omega_{m}^{-0.3}$ line of constant $\ell_{A}$ (dotted). If we instead add the LRG information (which constrains $h \Omega_{m}^{0.93}$ via the $P(k)$ turnover scale and $h \Omega_{m}^{-0.37}$ via the acoustic oscillation scale ${ }^{5}$, the allowed region shrinks to the thick ellipse.

\footnotetext{
${ }^{4}$ To obtain this $\omega_{\mathrm{m}}$-constraint, we marginalized over $\ell_{A}$ by marginalizing over either $\Omega_{k}$ or $w$; Table III shows that these two approaches give essentially identical answers.

${ }^{5}$ The origin of these scalings can be understood as follows. The matter-radiation equality horizon scale $r_{\mathrm{eq}} \propto \omega_{\mathrm{m}}^{-1}$. The sound horizon scales as $r_{\mathrm{s}}\left(z_{\mathrm{eq}}\right) \propto \omega_{\mathrm{m}}^{-0.25}$ with a weak dependence on $\omega_{b}$ that is negligible in this context [92]. For the LRG mean redshift $z=0.35$, the power law fit $d_{A}\left(z, \Omega_{m}\right)=$ $0.3253\left(\Omega_{m} / 0.25\right)^{-0.065} c H_{0}^{-1} \propto h^{-1} \Omega_{m}^{-0.065}$ is quite good within our range of interest, accurate to within about $0.1 \%$ for $0.2<$ $\Omega_{m}<0.3$. For $z=1100$, the power law fit $d_{A}\left(z, \Omega_{m}\right) \approx$ $3.4374\left(\Omega_{m} / 0.25\right)^{-0.4} \mathrm{cH}_{0}^{-1} \propto h^{-1} \Omega_{m}^{-0.4}$ retains $0.1 \%$ accuracy for $0.19<\Omega_{m}<0.35$. The $P(k)$ turnover angle $\propto$ $r_{\mathrm{eq}} / d_{A}(0.35) \propto\left(h^{2} \Omega_{m}\right)^{-1} / h^{-1} \Omega_{m}^{-0.065}$ is therefore constant for $h \propto \Omega_{m}^{-0.93}$, the $P(k)$ acoustic angle $\propto r_{\mathrm{s}} / d_{A}(0.35) \propto$ $\left(h^{2} \Omega_{m}\right)^{-0.25} / h^{-1} \Omega_{m}^{-0.065}$ is constant for $h \propto \Omega_{m}^{0.37}$, and the $C_{\ell}$ acoustic angle $\propto r_{\mathrm{s}} / d_{A}\left(z_{\text {rec }}\right) \propto\left(h^{2} \Omega_{m}\right)^{-0.25} / h^{-1} \Omega_{m}^{-0.4}$ is constant for $h \propto \Omega_{m}^{-0.3}$.
}

These two independent pieces of horizontal information are seen to be not only beautifully consistent, but also complementary: the joint constraints are significantly tighter than those from using either separately. When going beyond vanilla models below, the thin CMB-only ellipse is of course no longer relevant, making the LRG constraints even more valuable.

\section{Spacetime geometry}

To zeroth order (ignoring perturbations), the spacetime geometry is simply the Friedmann-Robertson-Walker metric determined by the curvature $\Omega_{k}$ and the cosmic expansion history $H(z)$. The vanilla assumptions imply the special case of no curvature $\left(\Omega_{k}=0\right)$ and constant dark energy $(H(z)$ given by Eq. (8) with $X(z)=1)$.

Let us now spice up the vanilla model space by including spatial curvature $\Omega_{k}$ and a constant dark energy equation of state $w$ as free parameters, both to constrain them and to quantify how other constraints get weakened when dropping these vanilla assumptions.

\section{LRGs as a standard ruler at $z=0.35$}

Before constraining specific spacetime geometry parameters, let us review the relevant physics to intuitively understand what CMB and LRGs do and do not teach us about geometry. As discussed in the previous section, current CMB data accurately measure only a single number that is sensitive to the spacetime geometry information in $\Omega_{k}$ and $H(z)$. This number is the peak angular scale $\ell_{A}$, and it in turn depends on the four independent parameters $\left(\Omega_{m}, \Omega_{k}, w, h\right)$. ( $\Omega_{\Lambda}$ is of course not independent, fixed by the identity $\Omega_{\Lambda}=1-\Omega_{k}-\Omega_{m}$.) Since the sound horizon size $r_{\mathrm{s}}$ is now accurately known independently of spacetime geometry from $\mathrm{CMB}$ peak ratios, the $\mathrm{CMB}$ $\ell_{A}$-measurement provides a precise determination of the comoving angular diameter distance to the last scattering surface, $d_{A}\left(z_{\text {rec }}\right)$, thus allowing one function of $\left(\Omega_{m}, \Omega_{k}, w, h\right)$ to be accurately measured.

As emphasized in $[36,128,130,131]$, measuring the acoustic angular scale at low redshift in galaxy clustering similarly constrains a second independent combination of $\left(\Omega_{m}, \Omega_{k}, w, h\right)$, and measuring $d_{A}(z)$ at multiple redshifts with future redshift surveys and current and future SN Ia data can break all degeneracies and allow robust recovery of both $\Omega_{k}$ and the dark energy history $X(z)$. For the galaxy approach, the point is that leaving the early-universe physics $\left(\omega_{b}, \omega_{\mathrm{m}}, n_{s}\right.$, etc.) fixed, changing the spacetime geometry merely scales the horizontal axis of the angular power spectrum of galaxies at a given redshift $z$ as $d_{A}(z)$. More generally, as described in detail in [36], the main effect of changing the spacetime geometry is to shift our measured three-dimensional power spectrum horizontally by rescaling the $k$ axis. The $k$-scale for angular modes dilates as the comoving angular diameter distance $d_{A}(z)$ to the mean survey redshift $z \approx 0.35$, whereas that for 
radial modes dilates as $d\left(d_{A}\right) / d z=c / H(z)$ for the flat case. For small variations around our best-fit model, the change in $H(0.35)$ is about half that of the angular diameter distance. To model this, [36] treats the net dilation as the cube root of the product of the radial dilation times the square of the transverse dilation, defining the distance parameter

$$
d_{V}(z) \equiv\left[d_{A}(z)^{2} \frac{c z}{H(z)}\right]^{1 / 3}
$$

Using only the vertical WMAP peak height information as a prior on $\left(\omega_{b}, \omega_{d}, n_{s}\right)$, our LRG power spectrum gives the measurement $\quad d_{V}(0.35)=1.300 \pm 0.088 \mathrm{Gpc}, \quad$ which agrees well with the value measured in [36] using the LRG correlation function. It is this geometric LRG information that explains most of the degeneracy breaking seen in the Figs. 15-18 below.

As more LRG data become available and strengthen the baryon bump detection from a few $\sigma$ to $>5 \sigma$, this measurement should become even more robust, not requiring any $\omega_{\mathrm{m}}$-prior from WMAP peak heights.

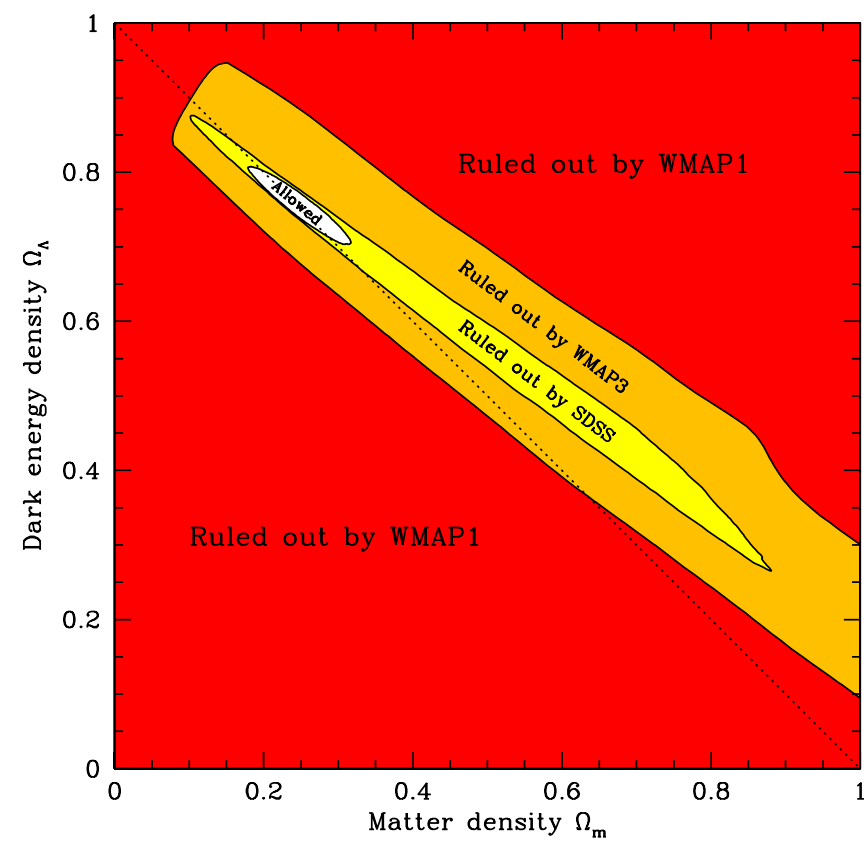

FIG. 15 (color online). $95 \%$ constraints in the $\left(\Omega_{m}, \Omega_{\Lambda}\right)$ plane. The large shaded regions are ruled out by WMAP1 (red/dark gray) and WMAP3 (orange/gray) when spatial curvature is added to the 6 vanilla parameters, illustrating the well-known geometric degeneracy between models that all give the same acoustic peak locations in the $\mathrm{CMB}$ power spectrum. The yellow/light gray region is ruled out when adding SDSS LRG information, breaking the degeneracy mainly by measuring the acoustic peak locations in the galaxy power spectrum. Models on the diagonal dotted line are flat, those below are open and those above are closed. Here the yellow banana has been cut off from below by an $h \gtrsim 0.4$ prior in the CosmoMC software.

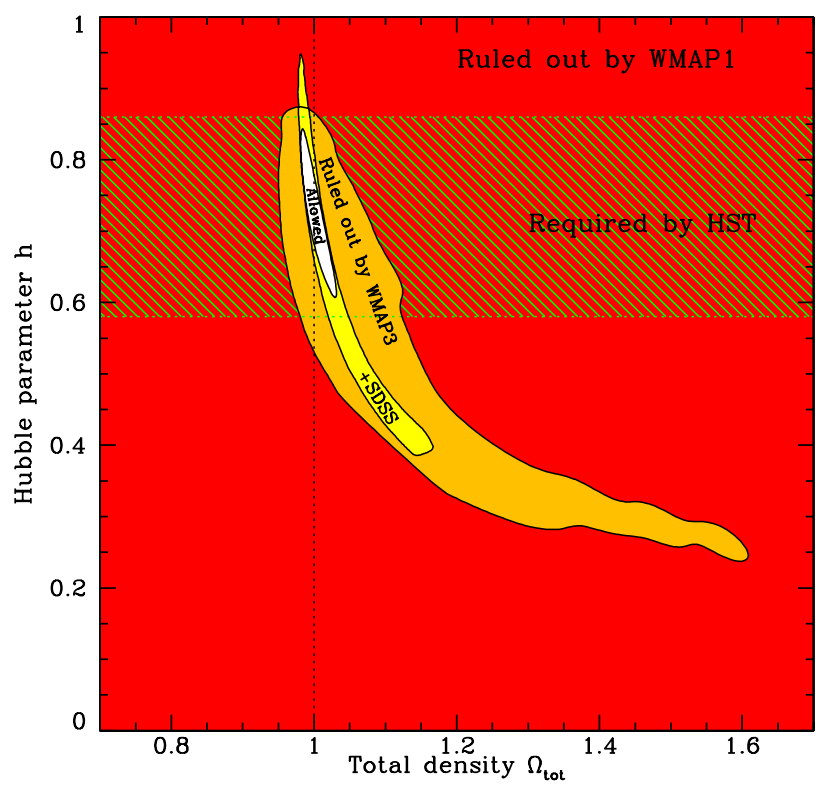

FIG. 16 (color online). $95 \%$ constraints in the $\left(\Omega_{\mathrm{tot}}, h\right)$ plane for 7-parameter curved models. The shaded red/dark gray region was ruled out by WMAP1 alone, and WMAP3 tightened these constraints (orange/gray region), illustrating that CMB fluctuations alone do not simultaneously show space to be flat and measure the Hubble parameter. The yellow/light gray region is ruled out when adding SDSS LRG information. Here the yellow banana has been artificially cut off for $h \geqslant 0.4$ by a hardwired prior in the CosmoMC software.

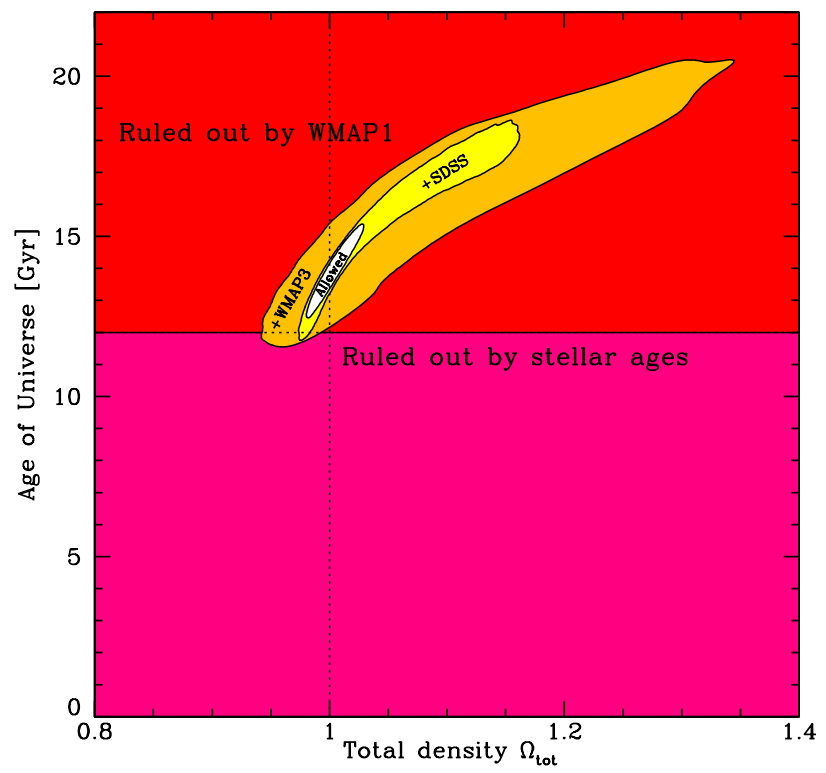

FIG. 17 (color online). $95 \%$ constraints in the $\left(\Omega_{\text {tot }}, t_{\text {now }}\right)$ plane for 7-parameter curved models. The shaded red/dark gray region is ruled out by WMAP1 alone, and WMAP3 tightened these constraints (orange/gray region), illustrating that CMB fluctuations do not simultaneously show space to be flat and measure the age of the Universe. The yellow/light gray region is ruled out when adding SDSS LRG information. The age limit $t_{\text {now }}>$ 12 Gyr shown is the $95 \%$ lower limit from white dwarf ages by [184]; for a review of recent age determinations, see [7]. 


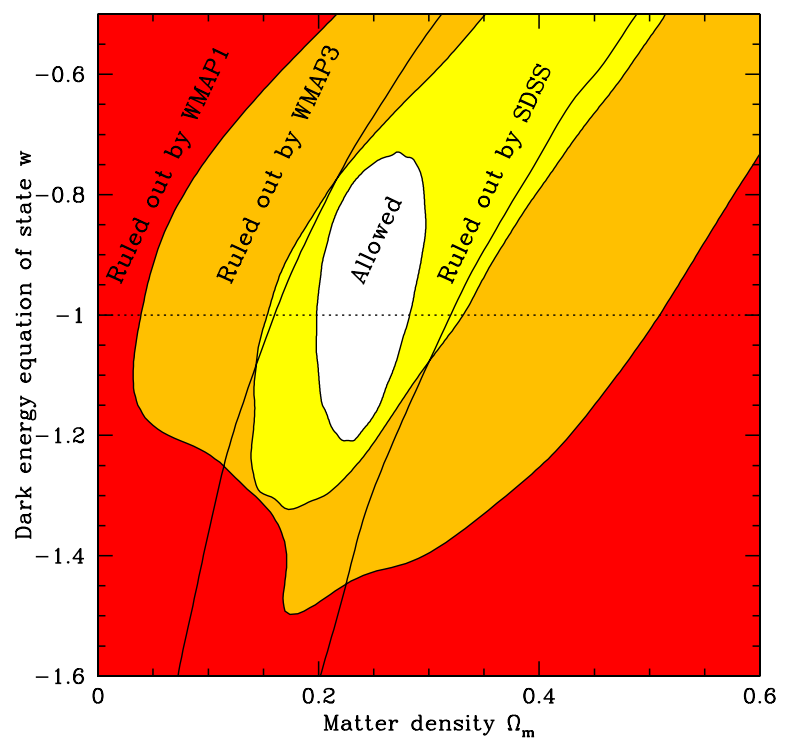

FIG. 18 (color online). $95 \%$ constraints in the $\left(\Omega_{m}, w\right)$ plane. The shaded red/gray region is ruled out by WMAP1 alone when the dark energy equation of state $w$ is added to the 6 vanilla parameters. The shaded orange/gray region is ruled out by WMAP3. The yellow/light gray region is ruled out when adding SDSS LRG information. The region not between the two black curves is ruled out by WMAP3 when dark energy is assumed to cluster.

\section{Spatial curvature}

Although it has been argued that closed inflation models require particularly ugly fine-tuning [132], a number of recent papers have considered nearly-flat models either to explain the low CMB quadrupole [133], in string theory landscape-inspired short inflation models, or for anthropic reasons $[94,134,135]$, so it is clearly interesting and worthwhile to continue sharpening observational tests of the flatness assumption. In the same spirit, measuring the Hubble parameter $h$ independently of theoretical assumptions about curvature and measurements of galaxy distances at low redshift provides a powerful consistency check on our whole framework.

Figs. 15-17 illustrate the well-known CMB degeneracies between the curvature $\Omega_{k} \equiv 1-\Omega_{\text {tot }}$ and dark energy $\Omega_{\Lambda}$, the Hubble parameter $h$, and the age of the universe $t_{\text {now }}$; without further information or priors, one cannot simultaneously demonstrate spatial flatness and accurately measure $\Omega_{\Lambda}, h$ or $t_{\text {now }}$, since the CMB accurately constrains only the single combination $\ell_{A}$. Indeed, the WMAP3 degeneracy banana extends towards even larger $\Omega_{\text {tot }}$ than these figures indicate; the plotted banana has been artificially truncated by a hardwired lower limit on $h$ in the CosmoMC software used to compute this particular MCMC.

Including our LRG information is seen to reduce the curvature uncertainty by about a factor of 5 , providing a striking vindication of the standard inflationary prediction $\Omega_{\mathrm{tot}}=1$. The physical reason for this LRG improvement is obvious from the thick ellipse in Fig. 14: WMAP vertical peak height information combined with LRG standard ruler information on $d_{V}(0.35)$ measures $\Omega_{m}$ rather independently of curvature.

Yet even with WMAP + LRG information, the figures show that a strong degeneracy persists between curvature and $h$, and curvature and $t_{\text {now }}$, leaving the measurement uncertainty on $h$ comparable with that from the HST key project [136]. If we add the additional assumption that space is exactly flat, then uncertainties shrink by factors around 4 and 10 for $h$ and $t_{\text {now }}$, respectively, still in beautiful agreement with other measurements.

In conclusion, within the class of almost flat models, the WMAP-only constraints on $h, t_{\text {now }}, \Omega_{\Lambda}$, and $\Omega_{\text {tot }}$ remain weak, and including our LRG measurements provides a huge improvement in precision.

\section{Dark energy}

Although we now know its present density fairly accurately, we still know precious little else about dark energy, and much interest is focused on understanding its nature. Assuming flat space, Table III and Fig. 18 show our constraints on constant $w$ for two cases: assuming that dark energy is homogeneous (does not cluster) and that it allows spatial perturbations (does cluster) as modeled in [7]. We see that adding $w$ as a free parameter does not significantly improve $\chi^{2}$ for the best fit, and all data are consistent with the vanilla case $w=-1$, with $1 \sigma$ uncertainties in $w$ in the $10 \%-30 \%$ range, depending on dark energy clustering assumptions.

As described above, the physical basis of these constraints is similar to those for curvature, since (aside from low- $\ell$ corrections from the late ISW effect and dark energy clustering), the only readily observable effect of the dark energy density history $X(z)$ is to alter $d_{A}\left(z_{\text {rec }}\right)$ and $d_{A}(0.35)$, and hence the CMB and LRG acoustic angular scales. (The dark energy history also affects fluctuation growth and hence the power spectrum amplitude, but we do not measure this because our analysis marginalizes over the galaxy bias parameter $b$.)

It has been argued (see, e.g., [137]) that it is inappropriate to assume $\Omega_{k}=0$ when constraining $w$, since there is currently no experimental evidence for spatial flatness unless $w=-1$ is assumed. We agree with this critique, and merely note that no interesting joint constraints can currently be placed on as many as four spacetime geometry parameters $\left(\Omega_{m}, \Omega_{k}, w, h\right)$ from WMAP and our LRG measurements alone, since they accurately constrain only the two combinations $d_{A}\left(z_{\text {rec }}\right)$ and $d_{V}(0.35)$. Other data such as SN Ia need to be included for this; [7] do this and obtain $w=-1.06_{-0.08}^{+0.13}$.

One can also argue, in the spirit of Occam's razor, that the fact that vanilla works so well can be taken as evidence against both $\Omega_{k} \neq 0$ and $w \neq-1$, since it would require a fluke coincidence for them to both have significantly non- 
vanilla values that conspire to lie on the same $d_{V}(0.35)$ and $d_{A}\left(z_{\text {rec }}\right)$ degeneracy tracks as the vanilla model.

\section{Inflation}

Inflation [138-142] remains the leading paradigm for what happened in the early universe because it can solve the flatness, horizon and monopole problems (see, e.g., [143]) and has, modulo minor caveats, successfully predicted that $\Omega_{\text {tot }} \approx 1, n_{s} \approx 1,|\alpha| \ll 1$, and $r \lesssim 1$ as well as the facts that the seed fluctuations are mainly Gaussian and adiabatic. For the ekpyrotic universe alternative [144], controversy remains about whether it can survive a "bounce" and whether it predicts $n_{s} \approx 1$ [145] or $n_{s} \approx 3$ [146].

In the quest to measure the five parameters $\left(Q, n_{s}-\right.$ $\left.1, \alpha, r, n_{t}\right)$ characterizing inflationary seed fluctuations, the first breakthrough was the 1992 COBE discovery that $Q \sim$ $10^{-5}$ and that the other four quantities were consistent with zero [147]. The second breakthrough is currently in progress, with WMAP3 suggesting $1-n_{s}>0$ at almost the $3 \sigma$ level $\left(1-n_{s}=0.049_{-0.015}^{+0.019}\right)$ [7]. This central value is in good agreement with classic (single slow-rolling scalar field) inflation models, which generically predict nonscale invariance in the ballpark $1-n_{s} \sim 2 / N \sim 0.04$, assuming that the number of e-foldings between the time horizon the observed fluctuations exit the horizon and the end of inflation is $50<N<60$ as per [148]. This central value of $n_{s}$ agrees well with numerous measurements in the recent literature (e.g., [149]); it is merely the error bars that have changed.

As illustrated in Fig. 19 and discussed in [7], $n_{s}=1$ becomes allowed if the tensor fluctuation parameter $r$ is included (as it clearly should be when constraining inflation models), but the "vanilla lite" Harrison-Zeldovich model $\left(n_{s}=1, r=0\right)$ remains ruled out. In contrast, the arguably simplest of all inflation models, a single slowrolling scalar field with potential $V(\phi) \propto \phi^{2}$, remains viable: it predicts $\left(n_{s}, r\right)=(1-2 / N, 8 / N) \approx$ $(0.96,0.15)$. The string-inspired " $N$-flation" model makes a similar prediction [150,151].

Our constraints on the inflation parameters $\left(Q, n_{s}, \alpha, r, n_{t}\right)$ in Table III and Fig. 19 are seen to confirm those reported in [7] - the main addition of our LRG analysis is simply to provide a clean way of tightening the WMAP-only constraints on both $\Omega_{\text {tot }}$ and $r$ (by factors of 5 and 2, respectively). Lyman $\alpha$ Forest (Ly $\alpha \mathrm{F}$ ) constraints provide valuable complementary information on smaller scales, constraining the running of the spectral index to vanish at the percent level $[39,40]$.

Since the WMAP3 announcement, there has been substantial discussion of how strong the evidence against Harrison-Zeldovch $\quad\left(n_{s}=1, \quad r=0\right)$ really is $[39,127,152-157]$. For example, the WMAP team marginalized over the SZ-amplitude on small scales, which lowered the $n_{s}$-estimate by about 0.01 , but did not model

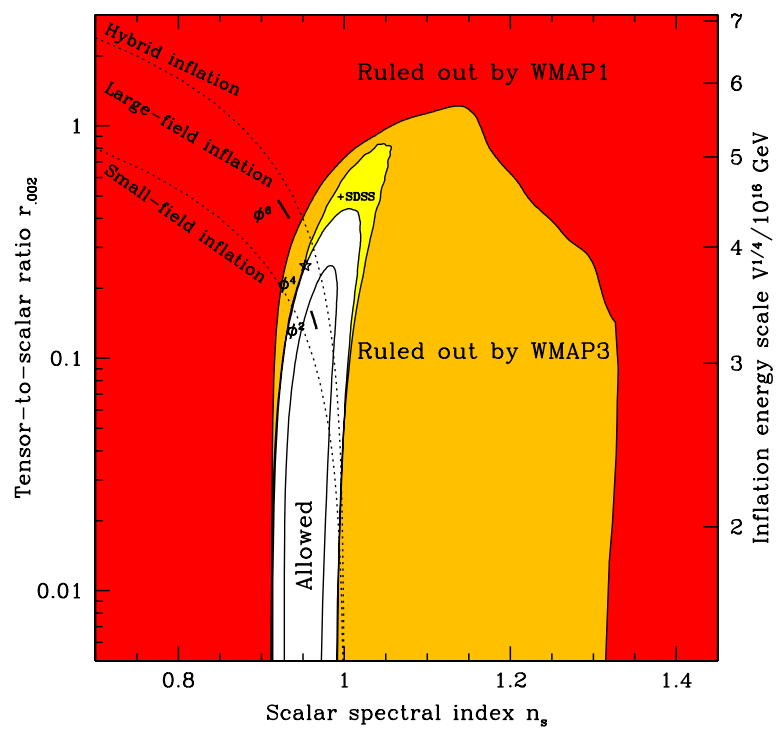

FIG. 19 (color online). $95 \%$ constraints in the $\left(n_{s}, r_{.002}\right)$ plane for 7-parameter tensor models (the vanilla parameters plus $r$ ). The large shaded regions are ruled out by WMAP1 (red/dark gray) and WMAP3 (orange/gray). The yellow/light gray region is ruled out when adding SDSS LRG information, pushing the upper limit on $r_{.002}$ down by a factor of 2 to $r_{.002}<0.33(95 \%)$. The solid black curve without shading shows the $68 \%$ limit. The two dotted lines delimit the three classes of inflation models known as small-field, large-field and hybrid models. Some single-field inflation models make highly specific predictions in this plane as indicated. From top to bottom, the figure shows the predictions for $V(\phi) \propto \phi^{6}$ (line segment; ruled out by CMB alone), $V(\phi) \propto \phi^{4}$ (star; a textbook inflation model; on verge of exclusion) and $V(\phi) \propto \phi^{2}$ (line segment; the eternal stochastic inflation model; still allowed). These predictions assume that the number of e-foldings between horizon exit of the observed fluctuations and the end of inflation is 64 for the $\phi^{4}$ model and between 50 and 60 for the others as per [148].

the CMB lensing effect, which would raise the $n_{s}$-estimate by a comparable amount [127]. It has also been argued that improved modeling of point source contamination increases the $n_{s}$-estimate [156]. Inclusion of smaller-scale CMB data and Ly $\alpha \mathrm{F}$ information clearly affects the significance as well. The bottom line is therefore that even modest improvements in measurement accuracy over the next few years can significantly improve our confidence in distinguishing between competing early-universe models-even without detecting $r>0$.

\section{E. Neutrinos}

It has long been known [158] that galaxy surveys are sensitive probes of neutrino mass, since they can detect the suppression of small-scale power caused by neutrinos streaming out of dark matter overdensities. For detailed discussion of post-WMAP3 astrophysical neutrino constraints, see [7,39,159-163].

Our neutrino mass constraints are shown in Fig. 20 and in the $M_{\nu}$-panel of Fig. 12, where we allow our standard 6 


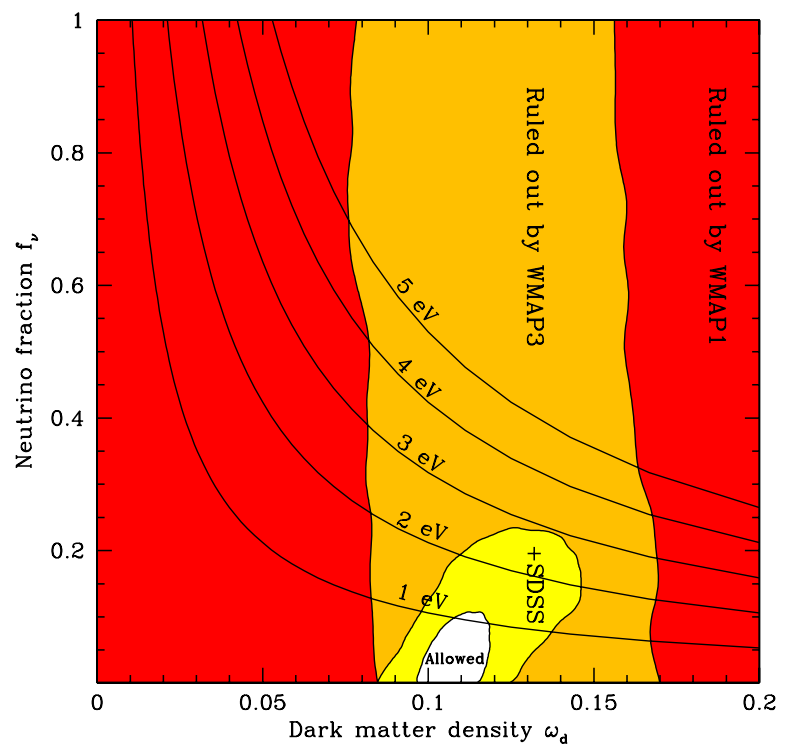

FIG. 20 (color online). $95 \%$ constraints in the $\left(\omega_{d}, f_{\nu}\right)$ plane. The large shaded regions are ruled out by WMAP1 (red/dark gray) and WMAP3 (orange/gray) when neutrino mass is added to the 6 vanilla parameters. The yellow/light gray region is ruled out when adding SDSS LRG information. The five curves correspond to $M_{\nu}$, the sum of the neutrino masses, equaling 1, $2,3,4$, and $5 \mathrm{eV}$, respectively-barring sterile neutrinos, no neutrino can have a mass exceeding $\sim M_{\nu} / 3 \approx 0.3 \mathrm{eV}(95 \%)$.

vanilla parameters and $f_{\nu}$ to be free. ${ }^{6}$ Assuming three active neutrinos with standard freeze-out abundance, we obtain a $95 \%$ upper limit $M_{\nu}<0.9 \mathrm{eV}$, so combining this with the atmospheric and solar neutrino oscillation results $[164,165]$, which indicate small mass differences between the neutrino types, implies that none of the three masses can exceed $M_{\nu} / 3 \approx 0.3 \mathrm{eV}$. In other words, the heaviest neutrino (presumably in a hierarchical model mostly a linear combination of $\nu_{\mu}$ and $\nu_{\tau}$ ) would have a mass in the range $0.04-0.3 \mathrm{eV}$.

If one is willing to make stronger assumptions about the ability to model smaller-scale physics, notably involving the $\operatorname{Ly} \alpha \mathrm{F}$, one can obtain the substantially sharper upper bound $M_{\nu}<0.17 \mathrm{eV}$ [39]. However, it should be noted that [39] also find that these same assumptions rule out the standard model with three active neutrino species at $2.5 \sigma$, preferring more than three species.

\section{F. Robustness to data details}

Above, we explored in detail how our cosmological parameter constraints depend on assumptions about physics in the form of parameter priors $\left(\Omega_{k}=0, w=-1\right.$, etc.). Let us now discuss how sensitive they are to details related to data modeling.

\footnotetext{
${ }^{6}$ It has been claimed that the true limits on neutrino masses from the WMAP1 (but not WMAP3) CMB maps are tighter than represented in these figures $[37,160,161]$.
}

\section{CMB modeling issues}

With any data set, it is prudent to be extra cautious regarding the most recent additions and the parts with the lowest signal-to-noise ratio. In the WMAP case, this suggests focusing on the $T$ power spectrum around the third peak and the large-scale $E$-polarization data, which as discussed in Sec. IV B 1 were responsible for tightening and lowering the constraints on $\omega_{\mathrm{m}}$ and $\tau$, respectively.

The large-scale $E$-polarization data appear to be the most important area for further investigation, because they are single-handedly responsible for most of the dramatic WMAP3 error bar reductions, yet constitute only a $3 \sigma$ detection after foregrounds an order of magnitude larger have been subtracted from the observed polarized CMB maps [2]. As discussed in [127] and Sec. IV B 1, all the WMAP3 polarization information is effectively compressed into the probability distribution for $\tau$, since using the prior $\tau=0.09 \pm 0.03$ instead of the polarized data leaves the parameter constraints essentially unchanged. This error bar $\Delta \tau=0.03$ found in [7] and Table II reflects only noise and sample variance and does not include foreground uncertainties. If future foreground modeling increases this error bar substantially, it will reopen the vanilla banana degeneracy described in [33]: Increasing $\tau$ and $A_{s}$ in such a way that $A_{\text {peak }} \equiv A_{s} e^{-2 \tau}$ stays constant, the peak heights remain unchanged and the only effect is to increase power on the largest scales. The large-scale power relative to the first peak can then be brought back down to the observed value by increasing $n_{s}$, after which the second peak can be brought back down by increasing $\omega_{b}$. Since quasar observations of the Gunn-Peterson effect allow $\tau$ to drop by no more than about $1 \sigma(0.03)[166,167]$, the main change possible from revised foreground modeling is therefore that $\left(\tau, \Omega_{\Lambda}, \omega_{d}, \omega_{b}, A_{s}, n_{s}, h\right)$ all increase together [33]. For a more detailed treatment of these issues, see [168].

A separate issue is that, as discussed in Sec. IV D, reasonable changes in the CMB data modeling can easily increase $n_{s}$ by of order 0.01 [39,127,152-154,156], weakening the significance with which the Harrison-Zeldovich model $\left(n_{s}=1, r=0\right)$ can be ruled out.

With the above-mentioned exceptions, parameter measurements now appear rather robust to WMAP modeling details. We computed parameter constraints using the WMAP team chains available on the LAMBDA archive. We created our own chains using the CosmoMC package [108] for the vanilla case (of length 310817 ) as a crosscheck and for the case with curvature (of length 226456) since this was unavailable on LAMBDA. The parameter constraints were in excellent agreement between these two vanilla chains. For a fair comparison between WMAP team and CosmoMC-based chains, the best-fit $\chi^{2}$ values listed in Table III have been offset-calibrated so that they all give the same value for our best-fit vanilla model. 


\section{LRG modeling issues}

Since we marginalize over the overall amplitude of LRG clustering via the bias parameter $b$, the LRG power spectrum adds cosmological information only through its shape. Let us now explore how sensitive this shape is to details of the data treatment. A popular way to parametrize the power spectrum shape in the literature has been in terms of the two parameters $\left(\Omega_{m}, f_{b}\right)$ shown in Fig. 21, where $f_{b} \equiv \Omega_{b} / \Omega_{m}$ is the baryon fraction. Since we wish to use $\left(\Omega_{m}, f_{b}\right)$ merely to characterize this shape here, not for constraining cosmology, we will ignore all CMB data and restrict ourselves to vanilla models with $n_{s}=1, h=$ 0.72 and $A_{s}=1$, varying only the four parameters $\left(\Omega_{m}, f_{b}, b, Q_{\mathrm{nl}}\right)$. Figure 21 suggest that for vanilla models, the two parameters $\left(\Omega_{m}, f_{b}\right)$ do in fact capture the bulk of this shape information, since the WMAP + LRG joint constraints from our full 6-parameter analysis are seen to be essentially the intersection of the WMAP and "ALL LRG" allowed regions in the $\left(\Omega_{m}, f_{b}\right)$-plane.

(a) Sensitivity to defogging - Fig. 21 shows good consistency between the power spectrum shapes recovered from the three radial subsamples. Let us now explore in more detail issues related to our nonlinear modeling. Our results were based on the measure-

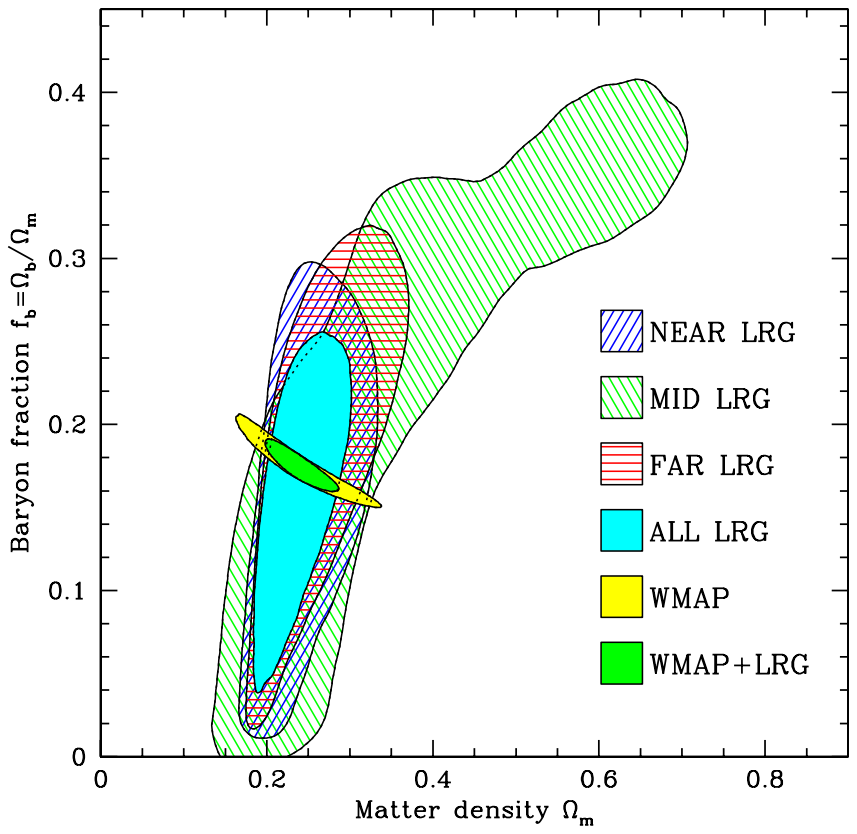

FIG. 21 (color online). The key information that our LRG measurements add to WMAP comes from the power spectrum shape. Parametrizing this shape by $\Omega_{m}$ and the baryon fraction $\Omega_{b} / \Omega_{m}$ for vanilla models with $n_{s}=1, h=0.72$, the $95 \%$ constraints above are seen to be nicely consistent between the various radial subsamples. Moreover, the WMAP + LRG joint constraints from our full 6-parameter analysis are seen to be essentially the intersection of the WMAP and "ALL LRG" allowed regions, indicating that these two shape parameters carry the bulk of the cosmologically useful LRG information. ment using FOG compression with threshold $\delta_{c}=$ 200 defined in [28]. Applied to the LRG sample alone, the FOG-compression algorithm (described in detail in [28]) finds about $20 \%$ of the LRGs in FOGs using this threshold; $77 \%$ of these FOGs contain two LRGs, $16 \%$ contain three, and $7 \%$ contain more than three. Thus not all LRGs are brightest cluster galaxies that each reside in a separate dark matter halo. Figure 22 shows a substantial dependence of $P(k)$ on this $\delta_{c}$ identification threshold for $k \gtrsim 0.1 \mathrm{~h} / \mathrm{Mpc}$. This is because FOGs smear out galaxy clusters along the line of sight, thereby strongly reducing the number of very close pairs, suppressing the small-scale power. Figure 22 shows that on small scales, the approximate scaling $P(k) \times$ ${ }_{\sim}^{\propto} k^{-1.3}$ seen for our default FOG compression matches the well-known correlation function scaling $\xi(r)_{\sim}^{\propto} r^{-1.7}$, which also agrees with the binding energy considerations of [169]. Fitting linear power spectra to these $P(k)$ curves would clearly give parameter constraints strongly dependent on $\delta_{c}$, with less aggressive FOG-removal (a higher threshold $\delta_{c}$ ) masquerading as lower $\Omega_{m}$. Using our nonlinear modeling, however, we find that $\delta_{c}$ has almost no effect on the cosmological parameters, with the change seen in Fig. 22 being absorbed by a change in the $Q_{\mathrm{nl}}$-parameter. For the three cases $\delta_{c}=(100200337)$, our above-mentioned 4parameter fits give highly stable best-fit values

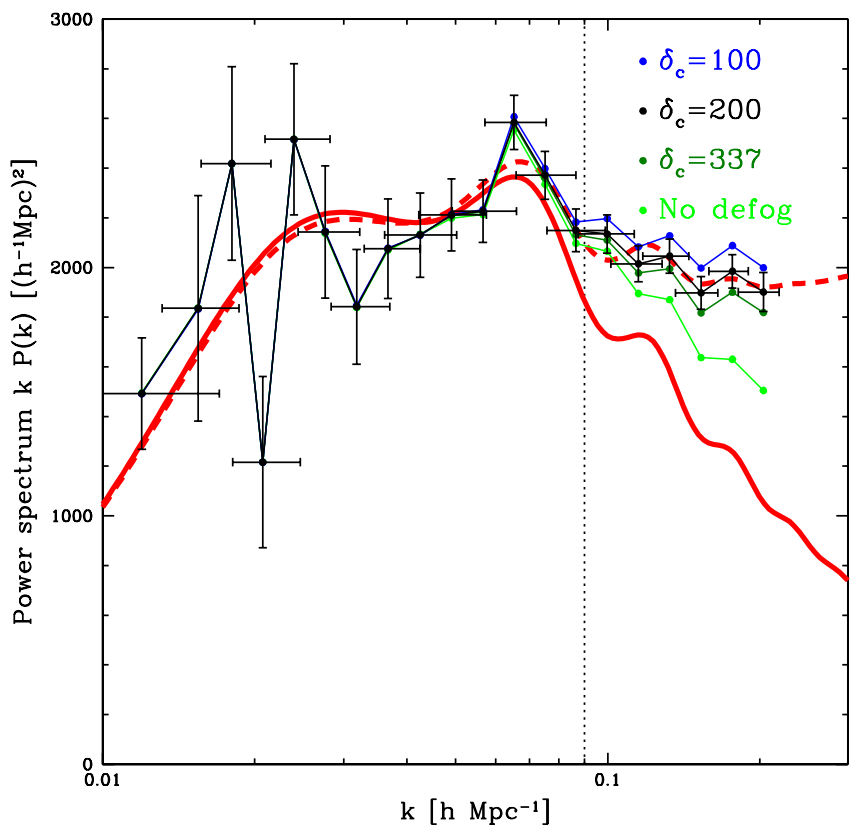

FIG. 22 (color online). Effect of finger-of-god (FOG) compression. Raising the FOG-compression threshold $\delta_{c}$ means that fewer FOGs are identified and compressed, which suppresses small-scale power while leaving the large-scale power essentially unchanged. 
$\Omega_{m}=(0.244,0.242,0.243)$ and $f_{b}=(0.168,0.169$, $0.168)$ together with the strongly varying best-fit values $Q_{\mathrm{nl}}=(27.0,30.9,34.2)$. If we fix the baryon density at the best-fit WMAP3 value and vary only the three parameters $\left(\Omega_{m}, b, Q_{\mathrm{nl}}\right)$, the corresponding results are $\Omega_{m}=(0.246,0.243,0.244)$ and $Q_{\mathrm{nl}}=$ $(27.1,31.0,34.3)$. Note that the cosmological parameter values do not show a rising or falling trend with $\delta_{c}$. For comparison, the $1 \sigma$ uncertainty on $\Omega_{m}$ from Table II is $\Delta \Omega_{m} \approx 0.02$, an order of magnitude larger than these variations. In other words, the $Q_{\mathrm{nl}}$-parameter closely emulates the effect of changing $\delta_{c}$, so that marginalizing over $Q_{\mathrm{nl}}$ is tantamount to marginalizing over $\delta_{c}$, making our treatment rather robust to the modeling of nonlinear redshift distortions.

(b) Sensitivity to k-cutoff-This is all very reassuring, showing that our cosmological constraints are almost completely unaffected by major changes in the $k \gtrsim 0.1 \mathrm{~h} / \mathrm{Mpc}$ power spectrum. (The reason that we nonetheless perform the $Q_{\mathrm{nl}}$-marginalization is if course that we wish to immunize our results against any small nonlinear corrections that extend to $k \lessgtr$ $0.1 \mathrm{~h} / \mathrm{Mpc}$.) To further explore this insensitivity to nonlinearities, we repeat the above analysis for the default $\delta_{c}=200$ case, including measurements for $0.01 h / \mathrm{Mpc} \leq k \leq k_{\max }$, and vary the upper limit $k_{\max }$. We apply a prior $0 \leq Q_{\mathrm{nl}} \leq 50$ to prevent unphysical $Q_{\mathrm{nl}}$-values for small $k_{\max }$-values (where $Q_{\mathrm{nl}}$ becomes essentially unconstrained). If no nonlinear modeling is performed, then as emphasized in [43], the recovered value of $\Omega_{m}$ should increase with $k_{\max }$ as nonlinear effects become important. In contrast, Fig. 23 shows that with our nonlinear modeling, the recovered $\Omega_{m}$-value is strikingly in-

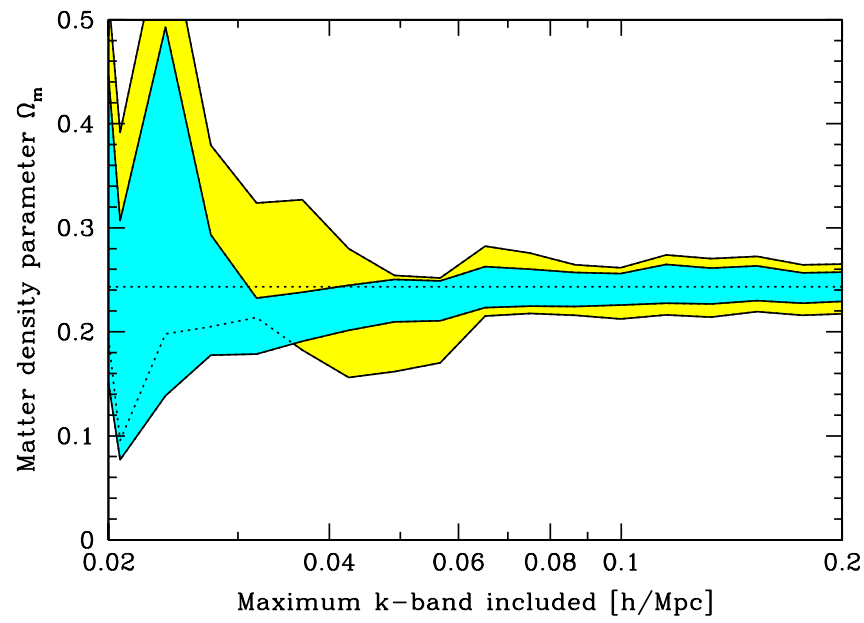

FIG. 23 (color online). $1 \sigma$ constraints on $\Omega_{m}$ as a function of the largest $k$-band included in the analysis. The yellow band shows the result when marginalizing over the baryon density $\omega_{b}$, the thinner cyan/gray band shows the result when fixing $\omega_{b}$ at the best-fit WMAP3 value. sensitive to $k_{\max }$. For $k_{\max } \ll 0.07 h / \mathrm{Mpc}$, the constraints are weak and fluctuate noticeably as each new band power is included, but for $k_{\max }$ beyond the first baryon bump at $k \sim 0.07 \mathrm{~h} / \mathrm{Mpc}$, both the central value and the measurement uncertainty remain essentially constant all the way out to $k_{\max }=$ $0.2 h / \mathrm{Mpc}$.

The above results tells us that, to a decent approximation, our $k \gtrsim 0.1 / \mathrm{Mpc}$ data are not contributing information about cosmological parameters, merely information about $Q_{\mathrm{nl}}$. Indeed, the error bar $\Delta \Omega_{m}$ is larger when using $k<0.2 \mathrm{~h} / \mathrm{Mpc}$ data and marginalizing over $Q_{\mathrm{nl}}$ then when using merely $k<$ $0.09 \mathrm{~h} / \mathrm{Mpc}$ data and fixing $Q_{\mathrm{nl}}$. In other words, our cosmological constraints come almost entirely from the LRG power spectrum shape at $k \lesssim$ $0.1 \mathrm{~h} / \mathrm{Mpc}$.

(c) Comparison with other galaxy $P(k)$-measurements - Let us conclude this section by briefly comparing with $\Omega_{m}$-values obtained from other recent galaxy clustering analyses.

Our WMAP3 + LRG measurement $\Omega_{m}=$ $0.24 \pm 0.02$ has the same central as that from WMAP3 alone [7], merely with a smaller error bar, and the most recent $2 \mathrm{dFGRS}$ team analysis also prefers $\Omega_{m} \approx 0.24$ [37]. This central value is $1.5 \sigma$ below the result $\Omega_{m}=0.30 \pm 0.04$ reported from WMAP1 + SDSS main-sample galaxies in [33]; part of the shift comes from the lower third peak in WMAP3 as discussed in Sec. IV B. PostWMAP3 results are also consistent with ours. Analysis of an independent SDSS LRG sample with photometric redshifts gave best-fit $\Omega_{m}$-values between 0.26 and 0.29 depending on binning [41], while an independent analysis including acoustic oscillations in SDSS LRGs and main-sample galaxies preferred $\Omega_{m} \approx 0.256$ [170].

The galaxy power spectra measured from the abovementioned data sets are likely to be reanalysed as nonlinear modeling methods improve. This makes it interesting to compare their statistical constraining power. [41] do so by comparing the error bar $\Delta \Omega_{m}$ from fitting two-parameter $\left(\Omega_{m}, b\right)$-models to all $k \leq 0.2 h / \mathrm{Mpc}$ data, with all other parameters, including $Q_{\mathrm{nl}}$ or other nonlinear modeling parameters, fixed at canonical best-fit values. This gives $\Delta \Omega_{m} \approx$ 0.020 for $2 \mathrm{dFGRS}$ and $\Delta \Omega_{m} \approx 0.012$ for for the SDSS LRG sample with photometric redshifts [41]. Applying the same procedure to our LRGs yields $\Delta \Omega_{m}=0.007$. This demonstrates both the statistical power of our sample, and that our cosmological analysis has been quite conservative in the sense of marginalizing away much of the power spectrum information (marginalizing over $Q_{\mathrm{nl}}$ doubles the error bar to $\Delta \Omega_{m}=0.014$ ). 


\section{Other issues}

A fortunate side effect of improved cosmological precision is that priors now matter less. Monte Carlo Markov chain generators usually assume a uniform Bayesian prior in the space of its "work parameters." For example, if two different papers parametrize the fluctuation amplitude with $A_{s}$ and $\ln A_{s}$, respectively, they implicitly assign $A_{s}$-priors that are constant and $\propto 1 / A_{s}$, respectively (the new prior picks up a factor from the Jacobian of the parameter transformation). Such prior differences could lead to substantial $(\sim 1 \sigma)$ discrepancies on parameter constraints a few years ago, when some parameters were still only known to a factor of order unity. In contrast, Table II shows that most parameters are now measured with relative errors in the range $1 \%-10 \%$. As long as these relative measurement errors are $\ll 1$, such priors become unimportant: Since the popular reparametrizations in the literature and in Table II involve smooth functions that do not blow up except perhaps where parameters vanish or take unphysical values, the relative variation of their Jacobian across the allowed parameter range will be of the same order as the relative variation of the parameters $(\ll 1)$, i.e., approximately constant. Chosing a uniform prior across the allowed region in one parameter space is thus essentially equivalent to choosing a uniform prior across the allowed region of anybody else's favorite parameter space.

\section{CONCLUSIONS}

We have measured the large-scale real-space power spectrum $P(k)$ using luminous red galaxies in the Sloan Digital Sky Survey (SDSS) with narrow well-behaved window functions and uncorrelated minimum-variance errors. The results are publicly available in an easy-to-use form at http://space.mit.edu/home/tegmark/sdss.html.

This is an ideal sample for measuring the large-scale power spectrum, since its effective volume exceeds that of the SDSS main galaxy sample by a factor of 6 and that of the 2dFGRS by an order of magnitude. Our results are robust to omitting purely angular and purely radial density fluctuations and are consistent between different parts of the sky. They provide a striking model-independent confirmation of the predicted large-scale $\Lambda \mathrm{CDM}$ power spectrum. The baryon signature is clearly detected (at $3 \sigma$ ), and the acoustic oscillation scale provides a robust measurement of the distance to $z=0.35$ independent of curvature and dark energy assumptions.

Although our measured power spectrum provides independent cross-checks on $\Omega_{m}$ and the baryon fraction, in good agreement with WMAP, its main utility for cosmological parameter estimation lies in complementing $\mathrm{CMB}$ measurements by breaking their degeneracies; for example, Table III shows that it cuts error bars on $\Omega_{m}, \omega_{\mathrm{m}}$, and $h$ by about a factor of 2 for vanilla models (ones with a cosmological constant and negligible curvature, tensor modes, neutrinos and running spectral index) and by up to almost an order of magnitude when curvature, tensors, neutrinos or $w$ are allowed. We find that all these constraints are essentially independent of scales $k>$ $0.1 \mathrm{~h} / \mathrm{Mpc}$ and associated nonlinear complications.

Since the profusion of tables and figures in Sec. IV can be daunting to digest, let us briefly summarize them and discuss both where we currently stand regarding cosmological parameters and some outstanding issues.

\section{A. The success of vanilla}

The first obvious conclusion is that "vanilla rules OK." We have seen several surprising claims about cosmological parameters come and go recently, such as a running spectral index, very early reionization and cosmologically detected neutrino mass - yet the last two rows of Table III show that there is no strong evidence in the data for any nonvanilla behavior: none of the nonvanilla parameters reduces $\chi^{2}$ significantly relative to the vanilla case. The WMAP team made the same comparison for the CMBonly case and came to the same conclusion [7]. Adding a generic new parameter would be expected to reduce $\chi^{2}$ by about unity by fitting random scatter. Although WMAP alone very slightly favor spatial curvature, this preference disappears when SDSS is included. The only nonvanilla behavior that is marginally favored is running spectral index $\alpha<0$, although only at $1.6 \sigma$. This persistent success of the vanilla model may evoke disturbing parallels with the enduring success of the standard model of particle physics, which has frustrated widespread hopes for surprises. However, the recent evidence for $n_{s}<1$ represents a departure from the $n_{s}=1$ "vanilla lite" model that had been an excellent fit ever since COBE [147], and as we discuss below, there are good reasons to expect further qualitative progress soon.

\section{B. Which assumptions matter?}

When quoting parameter constraints, it is important to know how sensitive they are to assumptions about both data sets and priors. The most important data assumptions discussed in Section IV F are probably those about polarized $\mathrm{CMB}$ foreground modeling for constraining $\tau$ and those about nonlinear galaxy clustering modeling for constraining the power spectrum shape. The effect of priors on other parameters is seen by comparing the seven columns of Table III, and the effect of including SDSS is seen by comparing odd and even rows.

WMAP alone has robustly nailed certain parameters so well that that neither adding SDSS information nor changing priors have any significant effect. Clearly in this camp are the baryon density $\omega_{b}$ (constrained by WMAP evenodd peak ratios) and the reionization optical depth $\tau$ (constrained by WMAP low- $\ell E$-polarization); indeed, Table 1 in [39] shows that adding $\operatorname{Ly} \alpha \mathrm{F}$ and other CMB and LSS data does not help here either. The spectral index $n_{s}$ is also in this nailed-by-WMAP category as long as we assume 
that $\alpha$ is negligible; generic slow-roll inflation models predict $|\alpha| \lesssim 10^{-3}$, well below the limits of detectability with current data sets.

For many other parameters, e.g., $\Omega_{m}, h$, and $t_{\text {now }}$, the WMAP-only constraints are extremely sensitive to priors, with the inclusion of SDSS information tightening them by factors $2-10$. The prior assumptions of the vanilla model $\left(\Omega_{k}=r=f_{\nu}=\alpha=0, w=-1\right)$ matter a lot with WMAP alone, and when one of them is dropped, the best-fit values of $\Omega_{m}$ and $h$ are typically very different, with much larger errors. These assumptions no longer matter much when SDSS is included, greatly simplifying the caveat list that the cautious cosmologist needs to keep in mind. This is quite different from the recent past, when the joint constraints from older WMAP and SDSS data were sensitive to prior assumptions such as spatial flatness [33]; a major reason for this change is clearly the SDSS measurement of the baryon acoustic scale. Indeed, one of the most interesting results of our analysis is the strengthened evidence for a flat universe, with the constraint on $\Omega_{\text {tot }}$ tightening from $1.054_{-0.046}^{+0.064}$ (WMAP3 only) to $1.003_{-0.009}^{+0.010}$ (WMAP3 + SDSS).

In other words, large-scale cosmic clustering data now robustly constrain all the vanilla parameters, even when any one of $\left(f_{v}, \Omega_{k}, r, f_{\nu}, w\right)$ are included as in Table III. If $w$ is varied jointly with $\Omega_{k}$ (as it arguably should be [137]), one expects dramatically weakened constraints on the two (since two standard rulers cannot determine the three parameters $\left(w, \Omega_{k}, \Omega_{m}\right)$ ), but rather unaffected degradation for the rest.

\section{Other data}

Our cosmological parameter analysis has been very conservative, using the bare minimum number of data sets (two) needed to break all major degeneracies, and using measurements which mainly probe the large-scale linear regime. It is therefore interesting to compare our results with the complementary approach of [39] of pushing the envelope by using essentially all available data (including Ly $\alpha \mathrm{F}$, supernovae Ia and smaller-scale CMB experiments), which gives tighter constraints at the cost of more caveats. Comparing with the error bars in Table 1 of [39] shows that the additional data give merely modest improvements for $\left(\omega_{b}, \omega_{d}, n_{s}, r, h\right)$, a halving of the error bars on $\Omega_{\text {tot }}$ (still consistent with flatness), and great gains for $\alpha$ and $M_{\nu}$. These last two parameters are strongly constrained by the small-scale Ly $\alpha \mathrm{F}$ information, with [39] reporting $\alpha=-0.015 \pm 0.012$ and $M_{\nu} / 3<$ $0.06 \mathrm{eV}(95 \%)$, a factor of 6 below our constraint and bumping right up against the atmospheric lower bound $\sim 0.04 \mathrm{eV}$. On the other hand, the same analysis also rules out the standard model with three active neutrino species at $2.5 \sigma$ [39]; one can always worry about pushing the envelope too far by underestimating modeling uncertainties and systematics. [39] also highlight interesting tension at the
$2 \sigma$-level between the $\mathrm{Ly} \alpha \mathrm{F}$ and WMAP3 data regarding the fluctuation amplitude $\sigma_{8}$, and weak gravitational lensing may emerge as the decisive arbiter here, by directly pinning down the matter fluctuation amplitude independently of bias $[171,172]$.

\section{Future challenges}

The impressive improvement of cosmological measurements is likely to continue in coming years. For example, the SDSS should allow substantially better cosmological constraints from LRGs for several reasons. When the SDSS-II legacy survey is complete, the sky area covered should be about 50\% larger than the DR4 sample we have analyzed here, providing not only smaller error bars, but also narrower window functions as the gaps in Fig. 3 are filled in. Global photometric calibration will be improved [173]. Various approaches may allow direct measurements of the bias parameter $b$, e.g., galaxy lensing [174], higherorder correlations [175], halo luminosity modeling [176] and reionization physics [177]. A bias measurement substantially more accurate than our $11 \%$ constraint from redshift-space distortions would be a powerful degeneracy breaker. Figure 24 shows that our other galaxy nuisance parameter, $Q_{\mathrm{nl}}$, is somewhat degenerate with $\Omega_{m}$, so improved nonlinear modeling that reliably predicts the slight departure from linear theory in the quasilinear regime from smaller-scale data would substantially tighten our cosmological parameter constraints. More generally, any improved modeling that allows inclusion of higher $k$ will help.

As a result of such data progress in many areas, parameter constraints will clearly keep improving. How good is good enough? The baryon density $\omega_{b}$ is a parameter over which it is tempting to declare victory and move on: The

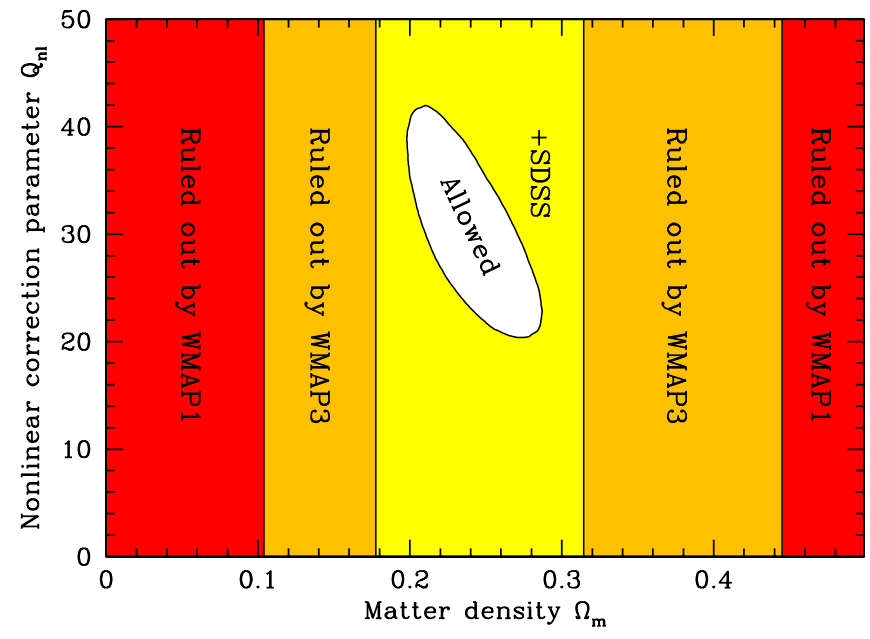

FIG. 24 (color online). $95 \%$ constraints in the $\left(\Omega_{m}, Q_{\mathrm{nl}}\right)$ plane for vanilla models. The shaded regions are ruled out by WMAP1 (red/dark gray), WMAP3 (orange/gray) and when adding SDSS LRG information. 
constraints on it from cosmic clustering are in good agreement, and are now substantially tighter than those from the most accurate competing technique against which it can be cross-checked (namely big bang nucleosynthesis), and further error bar reduction appears unlikely to lead to qualitatively new insights. In contrast, there are a number of parameters where cosmic clustering constraints are only now beginning to bump up against theory and other measurements, so that further sensitivity gains give great discovery potential. We have $\left(n_{s}, r, \alpha, \Omega_{k}\right)$ to test inflation, $M_{\nu}$ to cosmologically detect neutrino mass, $w$ and more generally $X(z)$ to constrain dark energy, and $\sigma_{8}$ to resolve tension between different cosmological probes.

Cosmology has now evolved from Alan Sandage's "search for two numbers" $\left(h, \Omega_{m}\right)$ to Alan Alexander Milne's "now we are six" $\left(h, \Omega_{b}, \Omega_{\mathrm{c}}, \sigma_{8}, n_{s}, \tau\right)$. Each time a nontrivial value was measured for a new parameter, nature gave up a valuable clue. For example, $\Omega_{\mathrm{c}}>0$ revealed the existence of dark matter, $\Omega_{\Lambda}>0$ revealed the existence of dark energy and the recent evidence for $n_{s}<1$ may sharpen into a powerful constraint on inflation. Milestones clearly within reach during the next few years include a measurement of $n_{s}<1$ at high significance and $M_{\nu}>0$ from cosmology to help uncover the neutrino mass hierarchy. If we are lucky and $r \sim 0.1$ (as suggested by classic inflation and models such as [150]), an $r>0$ detection will push the frontier of our ignorance back to $10^{-35} s$ and the GUT scale. Then there is always the possibility of a wild surprise such as $\Omega_{\text {tot }} \neq 1$, large $|\alpha|$, $X(z) \neq 1$, demonstrable non-Gaussianity, isocurvature contributions, or something totally unexpected. Our results have helped demonstrate that challenges related to survey geometry, bias and potential systematic errors can be overcome, giving galaxy clustering a valuable role to play in this ongoing quest for greater precision measurements of the properties of our universe.

\section{ACKNOWLEDGMENTS}

We thank Angelica de Oliveira-Costa, Kirsten A. Hubbard, Oliver Zahn, and Matias Zaldarriaga for helpful comments, and Dulce Gonçalves de Oliveira-Costa for ground support. We thank the WMAP team for making data and Monte Carlo Markov chains public via the Legacy Archive for Microwave Background Data Analysis (LAMBDA) at http://lambda.gsfc.nasa.gov, and Anthony Lewis and Sarah Bridle for making their CosmoMC software [108] available at http://cosmologist.info/cosmomc. Support for LAMBDA is provided by the NASA Office of Space Science. M.T. was supported by NASA Grants NAG5-11099 and NNG06GC55G, NSF Grants AST0134999, and 0607597, the Kavli Foundation, and the David and Lucile Packard Foundation, and the Research Corporation. D. J.E. was supported by NSF Grant AST0407200 and by the Alfred P. Sloan Foundation. Funding for the SDSS has been provided by the Alfred P. Sloan
Foundation, the Participating Institutions, the National Science Foundation, the U.S. Department of Energy, the National Aeronautics and Space Administration, the Japanese Monbukagakusho, the Max Planck Society, and the Higher Education Funding Council for England. The SDSS Web Site is http://www.sdss.org. The SDSS is managed by the Astrophysical Research Consortium for the Participating Institutions. The Participating Institutions are the American Museum of Natural History, Astrophysical Institute Potsdam, University of Basel, Cambridge University, Case Western Reserve University, University of Chicago, Drexel University, Fermilab, the Institute for Advanced Study, the Japan Participation Group, Johns Hopkins University, the Joint Institute for Nuclear Astrophysics, the Kavli Institute for Particle Astrophysics and Cosmology, the Korean Scientist Group, the Chinese Academy of Sciences (LAMOST), Los Alamos National Laboratory, the Max-Planck-Institute for Astronomy (MPIA), the Max-Planck-Institute for Astrophysics (MPA), New Mexico State University, Ohio State University, University of Pittsburgh, University of Portsmouth, Princeton University, the United States Naval Observatory, and the University of Washington.

\section{APPENDIX A: POWER SPECTRUM ESTIMATION DETAILS}

\section{Relation between methods for measuring the power spectrum and correlation function}

In this section, we clarify the relationship between different popular techniques for quantifying galaxy clustering with pair-based statistics, including correlation function estimation with the "DD - 2DR + RR" method [52,53] and power spectrum estimation with the FKP [19], FFT [26,29,30,38,43], and PKL [23,24,27,28,34,58] methods.

Suppose we have $N_{d}$ data points giving the comoving redshift-space position vectors $\mathbf{r}_{i}$ of galaxies numbered $i=$ $1, N_{d}$, and $N_{r}$ random points $\mathbf{s}_{i}$ from a mock catalog which has the same selection function $\bar{n}(\mathbf{r})$ as the real data. The number densities of data points and random points are then sums of Dirac $\delta$-functions:

$$
\begin{aligned}
& n_{d}(\mathbf{r})=\sum_{i=1}^{N_{d}} \delta\left(\mathbf{r}-\mathbf{r}_{i}\right), \\
& n_{r}(\mathbf{r})=\sum_{i=1}^{N_{r}} \delta\left(\mathbf{r}-\mathbf{s}_{i}\right) .
\end{aligned}
$$

By definition of the selection function $\bar{n}(\mathbf{r})$, the quantity

$$
\hat{\delta}(\mathbf{r}) \equiv \frac{n_{d}(\mathbf{r})-\alpha n_{r}(\mathbf{r})}{\bar{n}(\mathbf{r})},
$$

where $\alpha \equiv N_{d} / N_{r}$, is then an unbiased estimator of the underlying density fluctuation field $\delta(\mathbf{r})$ in the sense that $\langle\hat{\delta}\rangle=\delta$, where the averaging is over Poisson fluctuations 
as customary. Except for the PKL method, all techniques we will discuss take the same general form, weighting galaxy pairs in a form that depends only on the position of each galaxy and on the distance between the two, so we will now describe them all with a unified notation. (For an even more general pair-weighting formalism that also incorporates the PKL method, see [178].) As long as one uses $N_{r} \gg N_{d}$ random points, they will contribute negligible Poisson noise; their role is in effect to evaluate certain cumbersome integrals by Monte Carlo integration.

Let us define the quantity

$$
\hat{\xi}[f] \equiv \iint w(\mathbf{r}) \hat{\delta}(\mathbf{r}) w\left(\mathbf{r}^{\prime}\right) \hat{\delta}\left(\mathbf{r}^{\prime}\right) f\left(\left|\mathbf{r}-\mathbf{r}^{\prime}\right|\right) d^{3} r d^{3} r^{\prime} .
$$

Here $w(\mathbf{r})$ and $f(d)$ are the above-mentioned weight functions that depend on position and distance, respectively. As we will see, the "DD - 2DR + RR", FKP and FFT methods simply correspond to different choices of $w$ and $f$. Substituting Eqs. (A1)-(A3) into Eq. (A4), we find that

$$
\hat{\xi}[f]=\hat{\xi}_{d d}[f]-2 \hat{\xi}_{d r}[f]+\hat{\xi}_{r r}[f],
$$

where we have defined

$$
\begin{gathered}
\hat{\xi}_{d d}[f] \equiv \sum_{i=1}^{N_{d}} \sum_{j=1}^{N_{d}} \frac{w\left(\mathbf{r}_{i}\right) w\left(\mathbf{r}_{j}\right)}{\bar{n}\left(\mathbf{r}_{i}\right) \bar{n}\left(\mathbf{r}_{j}\right)} f\left(\left|\mathbf{r}_{i}-\mathbf{r}_{j}\right|\right), \\
\hat{\xi}_{d r}[f] \equiv \alpha \sum_{i=1}^{N_{d}} \sum_{j=1}^{N_{r}} \frac{w\left(\mathbf{r}_{i}\right) w\left(\mathbf{s}_{j}\right)}{\bar{n}\left(\mathbf{r}_{i}\right) \bar{n}\left(\mathbf{s}_{j}\right)} f\left(\left|\mathbf{r}_{i}-\mathbf{s}_{j}\right|\right), \\
\hat{\xi}_{r r}[f] \equiv \alpha^{2} \sum_{i=1}^{N_{r}} \sum_{j=1}^{N_{r}} \frac{w\left(\mathbf{s}_{i}\right) w\left(\mathbf{s}_{j}\right)}{\bar{n}\left(\mathbf{s}_{i}\right) \bar{n}\left(\mathbf{s}_{j}\right)} f\left(\left|\mathbf{s}_{i}-\mathbf{s}_{j}\right|\right) .
\end{gathered}
$$

As a first example, let us consider the FKP method [19]. This corresponds to [178]

$$
\begin{gathered}
f(d)=j_{0}(k d), \\
w(\mathbf{r}) \propto \frac{\bar{n}(\mathbf{r})}{1+\bar{n}(\mathbf{r}) P(k)},
\end{gathered}
$$

and turns $\hat{\xi}$ into the FKP estimator of the windowconvolved power spectrum $P(k)$. Here $j_{0}(x) \equiv \sin (x) / x$, $w$ is normalized so that $\int w(\mathbf{r})^{2} d^{3} r=1$ and $P$ is an a priori guess as to what the galaxy power spectrum is. For details, see [178] around Eqs. (25) and (56). The main point is that Fourier transforming $\hat{\delta}$ and averaging $|\hat{\delta}(\mathbf{k})|^{2}$ over a spherical shell in $k$-space gives the factor $\int e^{-i \mathbf{k} \cdot\left|\mathbf{r}-\mathbf{r}^{\prime}\right|} d \Omega_{k} / 4 \pi=j_{0}\left(k\left|\mathbf{r}-\mathbf{r}^{\prime}\right|\right)=f$. We apply this method to our LRG data and compare the results with those of [43] in Fig. 25, finding good agreement.

The FFT method $[26,29,30,38,43]$ is identical to the FKP method except for two simplifications: $P$ in Eq. (A10) is taken to be a $k$-independent constant and the density field is binned onto a three-dimensional grid to

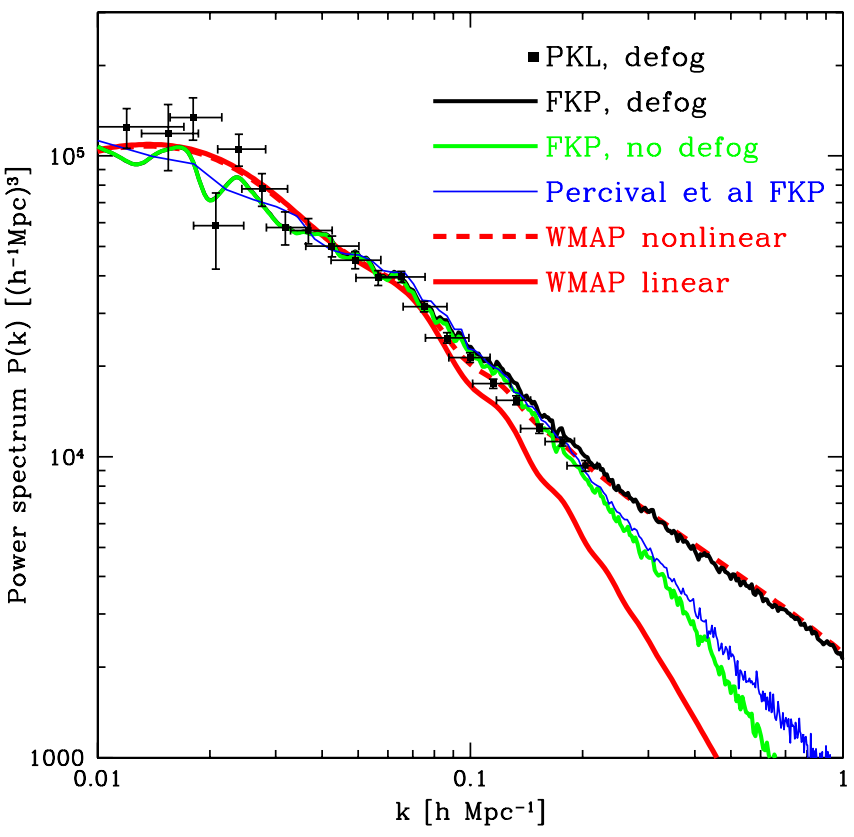

FIG. 25 (color online). Comparison of power spectrum estimation techniques. Our FKP measurement without defogging is seen to agree quite well with the measurement of [43] considering that the latter includes also main-sample galaxies with different $\beta$ and small-scale clustering properties. These curves cannot be directly compared with the PKL measurements or theoretical models, because they are not corrected for the effects of redshift distortions, window functions and the integral constraint; the qualitative agreement that is nonetheless seen is as good as one could expect given these caveats.

replace the time-consuming double sums above with a fast Fourier transform.

The "DD - 2DR + RR" method [52,53] estimates the correlation function $\xi(r)$ by the Landy-Szalay estimator

$$
\hat{\xi}_{\mathrm{LS}}=\frac{\hat{\xi}_{d d}-2 \hat{\xi}_{d r}+\hat{\xi}_{r r}}{\hat{\xi}_{r r}}
$$

which is often written informally as (DD $-2 \mathrm{DR}+$ $\mathrm{RR}) / \mathrm{RR}$. Here two common weighting choices in the literature are $w(\mathbf{r})=\bar{n}(\mathbf{r})$ [52] and $w(\mathbf{r})=\bar{n}(\mathbf{r}) /[1+$ $\bar{n}(\mathbf{r}) J]$ [53], where $J \equiv \int_{0}^{r} \xi\left(r^{\prime}\right) d^{3} r^{\prime}$ tends to be of the same order of magnitude as $P(k)$. To measure the binned correlation function using Eqs. (A6)-(A8), one thus sets $f(d)=1$ when $d$ is inside the bin and $f(d)=0$ otherwise.

These close relationships between the FKP, FFT and "DD - 2DR + RR" methods lead to interesting conclusions regarding all three methods.

First, it can be interesting for some applications to replace $J$ by $P$ when measuring the correlation function, using $w(\mathbf{r})=\bar{n}(\mathbf{r}) /[1+\bar{n}(\mathbf{r}) P]$, as was done for the analysis of the QDOT survey in [19] and for the LRG analysis in [36]. For instance, one could use a constant $P$ evaluated at the baryon wiggle scale if the goal is to measure the baryon bump in the correlation function. 
Second, there is an interesting equivalence between the methods. For reasons that will become clear below, let us refer to the numerator of Eq. (A11), $\hat{\xi}_{d d}-2 \hat{\xi}_{d r}+\hat{\xi}_{r r}$, as the "convolved" correlation function estimator and full expression $\hat{\xi}_{\text {LS }}$ as the "deconvolved" estimator. The information content in the convolved and decovolved estimators is clearly the same, since dividing by $\hat{\xi}_{r r}$ in Eq. (A11) is a reversible operation. Moreover, it is straightforward to show that the FKP estimator of $P(k)$ is simply the 3D Fourier transform of the convolved correlation function estimator as long as the same weighting function $w(r)$ is used for both. [179] also comment on this. (Note that this is a quite different statement from the well-known fact that $P(k)$ is the 3D Fourier transform of the correlation function $\xi(r)$.) This implies that the measured FKP power spectrum and the measured correlation function contain exactly the same information. In particular, it means that cosmological constraints from one are no better than cosmological constraints from the other, since they should be identical as long as window functions, covariance matrices, etc., are handled correctly. (An analogous correspondence for purely angular data is discussed in [180].) In contrast, the information content in the PKL measurement of the power spectrum is not identical; it uses a more general pair weighting than Eq. (A4) and by construction contains more cosmological information; a more detailed discussion of this point is given in Appendix A.3 in [181].

Third, this Fourier equivalence between the convolved correlation function estimator and the FKP power spectrum estimator sheds light on the fact that the deconvolved correlation function estimator $\hat{\xi}_{\text {LS }}$ is unbiased $\left(\langle\hat{\xi}\rangle_{\mathrm{LS}}(d)=\right.$ $\xi(d)$, the true correlation function), whereas the expectation value of the FKP estimator is merely the true power spectrum convolved with a so-called window function. This difference stems from the division by $\hat{\xi}_{r r}$ in Eq. (A11): Multiplication by $\hat{\xi}_{r r}$ in real space corresponds to convolution with the Fourier transform of $\hat{\xi}_{r r}$ (the window function) in Fourier space. The reason that one cannot deconvolve this windowing in Fourier space is that one cannot Fourier transform $\hat{\xi}_{\text {LS }}$, as it is completely unknown for large $d$-values that exceed all pair separations in the survey.

Fourth, this equivalence implies that gridding errors in the 3D FFT method (which become important at large $k$ [30]) can be completely eliminated by simply computing the correlation function with $w(\mathbf{r})=\bar{n}(\mathbf{r}) /[1+\bar{n}(\mathbf{r}) P]$ by summation over pairs and then transforming the convolved correlation function with the kernel $j_{0}(k r)$.

Figure 25 compares the LRG power spectra measured with the different techniques discussed above. A direct comparison between our PKL $P(k)$-measurement and that of [43] is complicated both by window function effects and by the fact that the latter was performed in redshift space without FOG compression, with SDSS MAIN galaxies mixed in with the LRG sample. To facilitate comparison, we performed our own FKP analysis using the direct summation method as described above, with constant $P=$ $30000\left(h^{-1} \mathrm{Mpc}\right)^{3}$ and $\alpha \approx 0.06$. This is seen to agree with the measurement of [43] to within a few percent for $0.04 h / \mathrm{Mpc}<k<0.2 h / \mathrm{Mpc}$ for the case of no defogging, with the remaining differences presumably due mainly to the inclusion of main-sample galaxies, particularly on small scales where nonlinear behavior becomes important. Figure 25 also shows that our defogged FKP measurements agree qualitatively between the PKL and FKP techniques, and that the FKP power spectrum continues to track our nonlinear WMAP model beautifully all the way out to $k=$ $1 h / \mathrm{Mpc}$ even though $Q_{\mathrm{nl}}$ was only fit to the $k<0.2 h / \mathrm{Mpc}$ PKL data.

An important caveat must be borne in mind when interpreting Fig. 25: The PKL points are constructed in such a way as to allow direct visual comparison with a model power spectrum [28], but the FKP and [43] curves are not, and should not be expected to fall right on top the PKL points or the best-fit cosmological model because they are not corrected for the effects of redshift distortions, window functions and the integral constraint. Redshift distortions should boost the FKP LRG curves slightly above the true real-space power spectrum (see Sec. A 3), and should boost the curve from Fig. 25 slightly more because the mainsample galaxies have a higher $\beta$ than the LRGs. The FKP window functions are broader than their PKL counterparts, and the steeper the power spectrum is, the more power leaks in from larger scales, causing the plotted measurements to lie above the true power spectrum. Finally, the integral constraint suppresses the plotted FKP power on the largest scales. In conclusion, the agreement seen in Fig. 25 is as good as one could expect given these many caveats.

\section{Numerical acceleration of the PKL method}

In this section, we describe a numerical improvement over the PKL power spectrum estimation method described in [28] that enables us to increase the number of modes from 4000 to 42000 .

The cosmological information content in a galaxy redshift survey, quantified by the Fisher information matrix [46,47,57], scales approximately as the effective volume $V_{\text {eff }}$ defined in Eq. (1), with error bars on cosmological parameters optimally measured from the survey scaling as $V_{\text {eff }}^{-1 / 2}$. However, actually extracting all this information in a numerically feasible way is far from trivial, contributing to the extensive literature on power spectrum estimation methods.

Our PKL method expands the galaxy density field in $N$ functions ("PKL modes") that probe successively smaller scales, and the number of modes needed to retain all information down to some length scale $\lambda=2 \pi / k$ is clearly of order $V_{\text {eff }} / \lambda^{3}$. In [28], $N=4000$ modes were used, and it was empirically determined that this retained essentially all information for $k \lessgtr 0.1 h / \mathrm{Mpc}$ with a grad- 
ual tapering off towards smaller scales. This was a convenient coincidence, since using $N \gg 4000$ becomes numerically painful: because of the many $N \times N$ matrix operations involved in the analysis, the disk usage is about 80 GB times $(N / 4000)^{2}$ and the CPU time required on a current workstation is about 20 days times $(N / 4000)^{3}$.

The effective volume of our LRG sample is about 10 times larger than that of the above-mentioned main galaxy analysis because the sky area covered has increased and because the sample is significantly deeper. To extract all the $k \lesssim 0.1 h / \mathrm{Mpc}$ information, we would therefore like to use about 10 times more modes, but without the analysis taking $10^{3}$ times longer $(\sim 50 \mathrm{yr})$.

We therefore combine the method of [33] with a divideand-conquer approach, performing a separate 2000-mode analysis on each of the 21 subvolumes described in Sec. II ( 3 radial $\times 7$ angular subsets) and combining the results with minimum-variance weighting (which, following the notation of [28], corresponds to simply summing both the F-matrices and the q-vectors). Although this combined analysis with its $21 \times 2000=42000$ modes becomes lossless in the information theory sense on scales substantially smaller than each of the 21 subvolumes, it destroys most of the information on scales comparable to these volumes, because the mean density in each volume is projected out (effectively marginalized over) [28]. It also becomes suboptimal on these largest scales because it neglects correlations between different subvolumes when optimizing the pair weighting. We therefore complement the combined analysis with a 4000-mode global analysis of the entire volume, which is optimal on the largest scales.

Both of these analyses produce uncorrelated band power estimators, and we use the first 8 (with $k<0.04 h / \mathrm{Mpc}$ ) from the global analysis and the remaining ones from the combined analysis. This splice point was chosen because the Fisher matrices show that the global analysis contains the most information (gives the smallest power spectrum error bars) for smaller $k$, and the combined analysis contains the most information for larger $k$. For the radial subsamples, the corresponding splice points are after bands 11 (NEAR), 10 (MID) and 8 (FAR). We confirm that, as the above scaling arguments suggest, the two analyses give essentially identical results in the intermediate $k$-range where they both retain virtually all the information. For example, the two analyses agree for band number 9 to about $0.7 \%$ in power, a difference which is completely negligible compared to the statistical error bars.

\section{Redshift-space distortion details}

As described in detail in [28], our PKL method produces three estimators $\left(\hat{P}_{\mathrm{gg}}(k), \hat{P}_{\mathrm{gv}}(k), \hat{P}_{\mathrm{vv}}(k)\right)$ of the galaxygalaxy, galaxy-velocity, and velocity-velocity power spectra $\left(P_{\mathrm{gg}}(k), P_{\mathrm{gv}}(k), P_{\mathrm{vv}}(k)\right)$. These estimators are uncorrelated, both with each other and between different $k$-bands, but not unbiased: the expectation value of $\hat{P}_{\mathrm{gg}}(k)$, say, includes contributions from all three power spectra. As explained in [28], we therefore construct our final power spectrum estimator $\hat{P}_{\mathrm{g}}$ as a linear combination of $\hat{P}_{\mathrm{gg}}(k)$, $\hat{P}_{\mathrm{gv}}(k)$, and $\hat{P}_{\mathrm{vv}}(k)$ that makes it an unbiased estimator of the real-space galaxy power spectrum $P_{\mathrm{gg}}(k)$. This linear combination corresponds to the process of marginalizing over the relative amplitudes of $P_{\mathrm{gv}}(k)$ and $P_{\mathrm{vv}}(k)$, which according to Eqs. (2) and (3) are $\beta r_{\mathrm{gv}}$ and $\beta^{2}$, respectively, so it can also be thought of as a marginalization over $\beta$ and $r_{\mathrm{gv}}$.

Two ways of forming this linear combination were explored in [28], referred to as the modeling method and the disentanglement method, respectively. The former corresponds to marginalizing over $\beta$ and $r_{\mathrm{gv}}$ globally, treating them as scale-independent constants, whereas the latter corresponds to treating them as arbitrary functions of $k$ and marginalizing over them separately for each $k$-band. We used the former approach for the "official" $P(k)$-measurement in [28] that was used for cosmological parameter estimation, and we make the same choice in the present paper, using only $k<0.09 \mathrm{~h} / \mathrm{Mpc}$ data to find the best-fitting values $\left(\beta, r_{\mathrm{gv}}\right) \approx(0.3,1)$. The latter approach is more conservative, at the price of producing much larger error bars.

To facilitate the interpretation of our thus-measured power spectrum $\hat{P}_{\mathrm{g}}(k)$, it is helpful to reexpress it in terms of multipoles of the redshift-space power spectrum. In the small-angle (distant observer) approximation where all galaxy pairs subtend a small-angle relative to the line of sight, $\left(P_{\mathrm{gg}}, P_{\mathrm{gv}}, P_{\mathrm{vv}}\right)$ reduce to simple linear combinations of the monopole, quadrupole and hexadecapole power spectra in redshift space $[182,183]$ :

$$
\left(\begin{array}{c}
P_{\mathrm{gg}}(k) \\
P_{\mathrm{gv}}(k) \\
P_{\mathrm{vv}}(k)
\end{array}\right)=\left(\begin{array}{ccc}
1 & -\frac{1}{2} & \frac{3}{8} \\
0 & \frac{3}{4} & -\frac{15}{8} \\
0 & 0 & \frac{35}{8}
\end{array}\right)\left(\begin{array}{c}
P_{0}^{\mathrm{s}}(k) \\
P_{2}^{\mathrm{s}}(k) \\
P_{4}^{\mathrm{s}}(k)
\end{array}\right) .
$$

Inverting Eq. (A12) gives

$$
\left(\begin{array}{c}
P_{0}^{\mathrm{s}}(k) \\
P_{2}^{\mathrm{s}}(k) \\
P_{4}^{\mathrm{s}}(k)
\end{array}\right)=\left(\begin{array}{ccc}
1 & \frac{2}{3} & \frac{1}{5} \\
0 & \frac{4}{3} & \frac{4}{7} \\
0 & 0 & \frac{8}{35}
\end{array}\right)\left(\begin{array}{c}
P_{\mathrm{gg}}(k) \\
P_{\mathrm{gv}}(k) \\
P_{\mathrm{vv}}(k)
\end{array}\right) .
$$

Equation (A12) tells us that, in the small-angle approximation, the disentanglement method would correspond to measuring $\left\langle\hat{P}_{\mathrm{g}}(k)\right\rangle=P_{0}^{\mathrm{s}}(k)-\frac{1}{2} P_{2}^{\mathrm{s}}(k)+\frac{3}{8} P_{4}^{\mathrm{s}}(k)=P_{\mathrm{gg}}(k)$. The corresponding weights for the modeling method are found by minimizing the variance among the class of all unbiased estimators, and thus depend on the detailed survey geometry, the shot noise level, etc. Empirically, we find $\left\langle\hat{P}_{\mathrm{g}}(k)\right\rangle \approx 0.8 P_{0}^{\mathrm{s}}(k)-0.07 P_{2}^{\mathrm{s}}(k)+0.006 P_{4}^{\mathrm{s}}(k)$, with the weights roughly independent of $k$. This can be intuitively understood from the fact that the estimators of $P_{2}^{\mathrm{s}}$ and $P_{4}^{\mathrm{s}}$ are much noisier than that for $P_{0}^{\mathrm{s}}$, and thus get assigned low statistical weight. If $P_{2}^{\mathrm{s}}$ and $P_{4}^{\mathrm{s}}$ were so noisy that they were 
discarded altogether, only the estimator of $P_{0}^{\mathrm{s}}$ would be used. The relation $P_{0}^{\mathrm{s}}(k)=\left(1+\frac{2}{3} r_{\mathrm{gv}} \beta+\frac{1}{5} \beta^{2}\right) P_{\mathrm{gg}}(k)$ following from Eq. (A13) would then give the simple estimator $\hat{P}_{\mathrm{g}}(k)=\hat{P}_{0}^{\mathrm{s}}(k) /\left(1+\frac{2}{3} r_{\mathrm{gv}} \beta+\frac{1}{5} \beta^{2}\right) \approx 0.8 \hat{P}_{0}^{\mathrm{s}}(k)$ for $\beta=0.3, r_{\mathrm{gv}}=1$, i.e., weights close to those we find empirically. Our measured uncertainty in this normalization factor $\left(1+\frac{2}{3} r_{\mathrm{gv}} \beta+\frac{1}{5} \beta^{2}\right)$ is about $3 \%$ (see Fig. 8), in good agreement with the exact numerical calculation described in [28], and this translates into an overall $3 \%$ calibration uncertainty of our measured power spectrum which is perfectly correlated between all $k$-bands.

The fact that the quantity measured by our power spectrum estimator $\hat{P}_{\mathrm{g}}(k)$ is so similar to the rescaled redshiftspace monopole spectrum is convenient, since it implies that nonlinear simulations of the redshift-space power spectrum (as discussed in Sec. IIID) should apply rather well to our results. However, it is important to keep in mind that our measurement $\hat{P}_{\mathrm{g}}(k)$ is a more accurate estimator of $P_{\mathrm{gg}}(k)$ than the rescaled redshift-space power spectrum would be, for several reasons. First, it never resorts to the small-angle approximation. Second, full account is taken of the fact that anisotropic survey geometry can skew the relative abundance of galaxy pairs around a single point that are aligned along or perpendicularly to the line-ofsight. These two caveats matter because $P_{2}^{\mathrm{s}}(k)$ and $P_{4}^{\mathrm{s}}(k)$ are undefined except in the small-angle limit, which could cause the correction factor $\left(1+\frac{2}{3} r_{\mathrm{gv}} \beta+\frac{1}{5} \beta^{2}\right)$ to be inaccurate on large scales. Finally, our estimator $\hat{P}_{\mathrm{g}}(k)$ by construction has smaller error bars than a standard FKP estimator of the redshift-space power spectrum, and one expects this advantage to be most important on the largest scales, comparable to and exceeding the thickness of the slices seen in Fig. 3.

\section{How spacetime geometry affects the power spectrum measurement}

We performed our power spectrum analysis in comoving three-dimensional space, with the conversion of redshifts into comoving distances performed for a fiducial flat $\Lambda$ CDM model with $\Omega_{m}=0.25$. As described in Sec. IV C 1, the conversion between redshift and comoving distance (measured in $h^{-1} \mathrm{Mpc}$ ) depends on the cosmo- logical parameters $\left(\Omega_{m}, \Omega_{\text {tot }}, w\right)$, so if a different fiducial model had been used for the conversion, then the inferred three-dimensional galaxy distribution in comoving coordinates would be radially dilated. As discussed in [36] and Sec. IV C 1, this would approximately dilate the dimensionless power spectrum $k^{3} P(k)$ by scaling the $k$ axis by a factor

$$
a \equiv \frac{d_{V}(z)}{d_{V}^{\text {fiducial }}(z)}
$$

where $d_{V}(z)$ is given by Eq. (9) and $z=0.35$ is the median survey redshift. For the parameter range allowed by WMAP3 and our LRG data,

$$
a \approx\left(\frac{\Omega_{m}}{0.25}\right)^{-0.087}\left(-w \Omega_{\mathrm{tot}}\right)^{0.19} .
$$

This means that the typical correction is very small: the rms scatter in the scaling factor $a$ is $0.7 \%$ for vanilla models, $1 \%$ for curved models and $3 \%$ for $w$-models. For example, increasing the fiducial $\Omega_{m}$-value by $25 \%$, from 0.24 to 0.30 , alters the scaling factor by $2 \%$ and, since the power spectrum turnover scale $\propto \Omega_{m}$, ignoring this correction could potentially bias the measured $\Omega_{m}$-value from 0.240 to 0.245 .

To be conservative, we nonetheless correct for this scaling effect in our likelihood software. Reanalyzing the galaxy data with the fiducial model replaced by the one to be tested would shift the measured $P(k)$ curve up to the left on a $\log -\log$ plot if $a>1$, with $k \mapsto k / a$ and $P \mapsto P a^{3}$. We therefore apply the opposite scaling $(k \mapsto k a$ and $P \mapsto$ $\left.P / a^{3}\right)$ to the theoretically predicted power spectrum $P(k)$ before computing its $\chi^{2}$ against our measurement power spectrum from Table I. We repeated our entire power spectrum analysis for $\Omega_{m}=0.30$ and confirmed that this scaling is accurate. Our likelihood software, which is available at http://space.mit.edu/home/tegmark/sdss/, evaluates $a$ exactly instead of using Eq. (A15).

In summary, the correction discussed in this section is quite small, especially since marginalizing over bias erases the effect of the $a^{3}$ amplitude shift, but we include it anyway to ensure that there is no bias on cosmological parameter estimates.
[1] G. Hinshaw et al., astro-ph/0603451.

[2] L. Page et al., astro-ph/0603450.

[3] S. Masi, astro-ph/0507509.

[4] J. Sievers et al., astro-ph/0509203.

[5] D. J. Eisenstein, W. Hu, and M. Tegmark, Astrophys. J. 518, 2 (1999).

[6] G. Efstathiou and J. R. Bond, Mon. Not. R. Astron. Soc.
304, 75 (1999).

[7] D. N. Spergel et al., astro-ph/0603449.

[8] J.P. Huchra, M. J. Geller, V. de Lapparent, and I. I. G. Corwin, Jr., Astrophys. J. Suppl. Ser. 72, 433 (1990).

[9] E. E. Falco et al., PASP 111, 438 (1999).

[10] S. A. Shectman et al., Astrophys. J. 470, 172 (1996).

[11] W. Saunders et al., Mon. Not. R. Astron. Soc. 317, 55 
(2000).

[12] M. C. Cooper et al., astro-ph/0607512.

[13] M. Colless et al., astro-ph/0306581.

[14] D. G. York et al., Astron. J. 120, 1579 (2000).

[15] M.S. Vogeley, C. Park, M. J. Geller, and J. P. Huchra, Astrophys. J. 391, L5 (1992).

[16] K. B. Fisher, M. Davis, M. A. Strauss, A. Yahil, and J. P. Huchra, Astrophys. J. 402, 42 (1993).

[17] C. Park, M.S. Vogeley, M. J. Geller, and J. P. Huchra, Astrophys. J. 431, 569 (1994).

[18] L. N. da Costa, M. S. Vogeley, M. J. Geller, J. P. Huchra, and C. Park, Astrophys. J. 437, L1 (1994).

[19] H. A. Feldman, N. Kaiser, and J. A. Peacock, Astrophys. J. 426, 23 (1994).

[20] H. Tadros and G. Efstathiou, Mon. Not. R. Astron. Soc. 276, L45 (1995).

[21] H. Tadros and G. Efstathiou, Mon. Not. R. Astron. Soc. 282, 1381 (1996).

[22] H. Lin et al., Astrophys. J. 471, 617 (1996).

[23] N. Padmanabhan, M. Tegmark, and A. J. S. Hamilton, Astrophys. J. 550, 52 (2001).

[24] A. J.S. Hamilton, M. Tegmark, and N. Padmanabhan, Mon. Not. R. Astron. Soc. 317, L23 (2000).

[25] A. J. S. Hamilton and M. Tegmark, Mon. Not. R. Astron. Soc. 330, 506 (2002).

[26] W. J. Percival et al., Mon. Not. R. Astron. Soc. 327, 1297 (2001).

[27] M. Tegmark, A. J. S. Hamilton, and Y. Xu, Mon. Not. R. Astron. Soc. 335, 887 (2002).

[28] M. Tegmark et al., Astrophys. J. 606, 702 (2004).

[29] S. Cole et al., Mon. Not. R. Astron. Soc. 362, 505 (2005).

[30] G. Hütsi, Astron. Astrophys. 449, 891 (2006).

[31] X. Wang, M. Tegmark, and M. Zaldarriaga, Phys. Rev. D 65, 123001 (2002).

[32] D. N. Spergel et al., Astrophys. J. Suppl. Ser. 148, 175 (2003).

[33] M. Tegmark et al., Phys. Rev. D 69, 103501 (2004).

[34] A. C. Pope et al., Astrophys. J. 607, 655 (2004).

[35] U. Seljak et al., Phys. Rev. D 71, 103515 (2005).

[36] D. J. Eisenstein et al., Astrophys. J. 633, 560 (2005).

[37] A. G. Sanchez, C. M. Bauch, W. J. Percival, N. D. Padilla, S. Cole, C. S. Frenk, and P. Norberg, Mon. Not. R. Astron. Soc. 366, 189 (2006).

[38] G. Hütsi, astro-ph/0604129.

[39] U. Seljak, A. Slosar, and P. McDonald, astro-ph/0604335.

[40] M. Viel, M. G. Haehnelt, and A. Lewis, Mon. Not. R. Astron. Soc. 370, L51 (2006).

[41] N. Padmanabhan et al., astro-ph/0605302.

[42] C. Blake, A. Collister, S. Bridle, and O. Lahav, astro-ph/ 0605303.

[43] W. J. Percival et al., astro-ph/0608636.

[44] J. Adelman-McCarthy et al. (to be published).

[45] M.S. Vogeley and A.S. Szalay, Astrophys. J. 465, 34 (1996).

[46] M. Tegmark, A. N. Taylor, and A. F. Heavens, Astrophys. J. 480, 22 (1997).

[47] M. Tegmark, Phys. Rev. Lett. 79, 3806 (1997).

[48] N. Kaiser, Mon. Not. R. Astron. Soc. 219, 785 (1986).

[49] D. J. Eisenstein et al., Astron. J. 122, 2267 (2001).

[50] M. A. Strauss et al., Astron. J. 124, 1810 (2002).

[51] G. Richards et al., Astron. J. 123, 2945 (2002).
[52] S. D. Landy and A. S. Szalay, Astrophys. J. 412, 64 (1993).

[53] A. J.S. Hamilton, Astrophys. J. 417, 19 (1993).

[54] E. Hawkins et al., Mon. Not. R. Astron. Soc. 346, 78 (2003).

[55] D. J. Eisenstein et al., Astrophys. J. 619, 178 (2005).

[56] I. Zehavi et al., Astrophys. J. 621, 22 (2005).

[57] K. Karhunen, Über lineare Methoden in der Wahrscheinlichkeitsrechnung (Kirjapaino oy. sana, Helsinki, 1947).

[58] A. J. S. Hamilton, astro-ph/0503604.

[59] J. A. Peacock and D. Nicholson, Mon. Not. R. Astron. Soc. 253, 307 (1991).

[60] C. Stoughton et al., Astron. J. 123, 485 (2002).

[61] J.E. Gunn, M. A. Carr, C. M. Rockosi, and M. Sekiguchi et al., Astron. J. 116, 3040 (1998).

[62] J.E. Gunn, W. A. Siegmund, E. J. Mannery, and E. J. Owen et al., Astron. J. 131, 2332 (2006).

[63] M. Fukugita, T. Ichikawa, J.E. Gunn, M. Doi, K. Shimasaku, and D.P. Schneider, Astron. J. 111, 1748 (1996).

[64] J. R. Pier et al., Astron. J. 125, 1559 (2003).

[65] R. H. Lupton, J. E. Gunn, Z. Ivezić, G. R. Knapp, S. Kent, and N. Yasuda, in Astronomical Data Analysis Software and Systems X, ASP Conf. Ser. No. 238, edited by F. R. Harnden, Jr., F. A. Primini, and H. E. Payne (Astron. Soc. Pac., San Francisco, 2001), p. 269.

[66] R. H. Lupton et al. (unpublished).

[67] D. W. Hogg, D. P. Finkbeiner, D. J. Schlegel, and J.E. Gunn, Astron. J. 122, 2129 (2001).

[68] J. A. Smith et al., Astron. J. 123, 2121 (2002).

[69] Z. Ivezić, R. H. Lupton, D. Schlegel et al., Astron. Nachr. 325, 583 (2004).

[70] D. Tucker, S. Kent, and M. S. Richmond et al. (unpublished).

[71] D. J. Schlegel, D. P. Finkbeiner, and M. Davis, Astrophys. J. 500, 525 (1998).

[72] M. R. Blanton, R. H. Lupton, F. M. Maley, N. Young, I. Zehavi, and J. Loveday, Astron. J. 125, 2276 (2003).

[73] A. Uomoto et al., Proc. SPIE Int. Soc. Opt. Eng. 5492, 1411 (2004).

[74] J. Adelman-McCarthy et al., Astrophys. J. Suppl. Ser. 162, 38 (2006).

[75] M. R. Blanton et al., Astron. J. 129, 2562 (2005).

[76] A. J. S. Hamilton and M. Tegmark, Mon. Not. R. Astron. Soc. 349, 115 (2004).

[77] J. A. Peacock and S. J. Dodds, Mon. Not. R. Astron. Soc. 267, 1020 (1994).

[78] J. N. Fry, Astrophys. J. 461, L65 (1996).

[79] M. Tegmark and P. J.E. Peebles, Astrophys. J. Lett. 500, L79 (1998).

[80] H. J. Mo and S. D. M. White, Mon. Not. R. Astron. Soc. 282, 347 (1996).

[81] M. Giavalisco et al., Astrophys. J. 503, 543 (1998).

[82] N. Kaiser, Mon. Not. R. Astron. Soc. 227, 1 (1987).

[83] A. Dekel and O. Lahav, Astrophys. J. 520, 24 (1999).

[84] U. Pen, Astrophys. J. 504, 601 (1998).

[85] A. J.S. Hamilton, Mon. Not. R. Astron. Soc. 322, 419 (2001).

[86] M. Blanton R., R. Cen, J. P. Ostriker, M. A. Strauss, and M. Tegmark, Astrophys. J. 531, 1 (2000).

[87] V. Wild et al., Mon. Not. R. Astron. Soc. 356, 247 (2005). 
[88] D. J. Eisenstein, H. Seo, and M. White, astro-ph/0604361.

[89] D. J. Eisenstein and W. Hu, Astrophys. J. 511, 5 (1999).

[90] H. Seo and D. J. Eisenstein, Astrophys. J. 633, 575 (2005).

[91] E. W. Kolb and M. S. Turner, The Early Universe (Addison Wesley, New York, 1990).

[92] W. Hu, astro-ph/0407158.

[93] M. Tegmark, J. Silk, and A. Blanchard, Astrophys. J. 420, 484 (1994).

[94] M. Tegmark and M. J. Rees, Astrophys. J. 499, 526 (1998).

[95] M. Tegmark, A. Vilenkin, and L. Pogosian, Phys. Rev. D 71, 103523 (2005).

[96] W. Hu, M. Fukugita, M. Zaldarriaga, and M. Tegmark, Astrophys. J. 549, 669 (2001).

[97] V. Springel, C.S. Frenk, and S.D.M. White, Nature (London) 440, 1137 (2006).

[98] E. Huff, A. E. Schultz, M. White, D. J. Schlegel, and M. S. Warren, astro-ph/0607061.

[99] J. Yoo et al. (unpublished).

[100] P. McDonald, astro-ph/0609413.

[101] R.E. Smith, R. Scoccimarro, and R. K. Sheth, astro-ph/ 0609547.

[102] S. Bashinsky and E. Bertschinger, Phys. Rev. D 65, 123008 (2002).

[103] N. Y. Gnedin and O. Y. Gnedin, Astrophys. J. 509, 11 (1998).

[104] N. Metropolis, A. W. Rosenbluth, M. N. Rosenbluth, A. H. Teller, and E. Teller, J. Chem. Phys. 21, 1087 (1953).

[105] W. K. Hastings, Biometrika 97, 57 (1970).

[106] W. R. Gilks, S. Richardson, and D. J. Spiegelhalter, Markov Chain Monte Carlo in Practice (Chapman \& Hall, London, 1996).

[107] N. Christensen, R. Meyer, L. Knox, and B. Luey, Classical Quantum Gravity 18, 2677 (2001).

[108] A. Lewis and S. Bridle, Phys. Rev. D 66, 103511 (2002).

[109] A. Slosar and M. Hobson, astro-ph/0307219.

[110] L. Verde et al., Astrophys. J. Suppl. Ser. 148, 195 (2003).

[111] M. Chu, M. Kaplinghat, and L. Knox, Astrophys. J. 596, 725 (2003).

[112] H. Sandvik, M. Tegmark, X. Wang, and M. Zaldarriaga, Phys. Rev. D 69, 063005 (2004).

[113] U. Seljak, and M. Zaldarriaga, Astrophys. J. 469, 437 (1996).

[114] A. Lewis and A. Challinor, Phys. Rev. D 66, 023531 (2002).

[115] M. Tegmark, A. Aguirre, M. J. Rees, and F. Wilczek, Phys. Rev. D 73, 023505 (2006).

[116] J. D. Barrow and F. J. Tipler, The Anthropic Cosmological Principle (Clarendon Press, Oxford, 1986).

[117] A. D. Linde, 300 Years of Gravitation, edited by S. Hawking and W. Israel (Cambridge University Press, Cambridge, 1987).

[118] S. Weinberg, Phys. Rev. Lett. 59, 2607 (1987).

[119] G. Efstathiou, Mon. Not. R. Astron. Soc. 274, L73 (1995).

[120] A. Vilenkin, Phys. Rev. Lett. 74, 846 (1995).

[121] H. Martel, P. R. Shapiro, and S. Weinberg, Astrophys. J. 492, 29 (1998).

[122] J. Garriga and A. Vilenkin, Phys. Rev. D 67, 043503 (2003).

[123] M. Tegmark, J. Cosmol. Astropart. Phys. 4 (2005) 1.

[124] A. Kosowsky, M. Milosavljevic, and R. Jimenez, Phys. Rev. D 66, 063007 (2002).
[125] R. Jimenez, L. Verde, H. Peiris, and A. Kosowsky, Phys. Rev. D 70, 023005 (2004).

[126] W. A. Fendt and B. Wandelt D., astro-ph/0606709.

[127] A. Lewis, astro-ph/0603753.

[128] Y. Wang and M. Tegmark, Phys. Rev. Lett. 92, 241302 (2004).

[129] Y. Wang and P. Mukherjee, astro-ph/0604051.

[130] D. J. Eisenstein, W. Hu, and M. Tegmark, Astrophys. J. Lett. 504, L57 (1998).

[131] C. Blake and K. Glazebrook, Astrophys. J. 594, 665 (2003).

[132] A. Linde, J. Cosmol. Astropart. Phys. 05, 2 (2003).

[133] G. Efstathiou, Mon. Not. R. Astron. Soc. 343, L95 (2003).

[134] A. D. Linde, Phys. Lett. B 351, 99 (1995).

[135] A. Vilenkin and S. Winitzki, Phys. Rev. D 55, 548 (1997).

[136] W. L. Freedman et al., Astrophys. J. 553, 47 (2001).

[137] E. L. Wright, astro-ph/0603750.

[138] A. Guth, Phys. Rev. D 23, 347 (1981).

[139] A. A. Starobinsky, Phys. Lett. B 91, 99 (1980).

[140] A. D. Linde, Phys. Lett. B 108, 389 (1982).

[141] A. Albrecht and P. J. Steinhardt, Phys. Rev. Lett. 48, 1220 (1982).

[142] A. D. Linde, Phys. Lett. B 129, 177 (1983).

[143] J. Bock et al., astro-ph/0604101.

[144] J. Khoury, B. Ovrut, P. J. Steinhardt, and N. Turok, Phys. Rev. D 64, 123522 (2001).

[145] J. Khoury, P. J. Steinhardt, and N. Turok, Phys. Rev. Lett. 91, 161301 (2003).

[146] P. Creminelli, A. Nicolis, and M. Zaldarriaga, Phys. Rev. D 71, 063505 (2005).

[147] G. F. Smoot et al., Astrophys. J. 396, L1 (1992).

[148] A. R. Liddle and S. M. Leach, Phys. Rev. D 68, 103503 (2003).

[149] C. McTavish et al., astro-ph/0507503.

[150] S. Dimopoulos, S. Kachru, J. McGreevy, and J. Wacker, hep-th/0507205.

[151] R. Easther and L. McAllister, J. Cosmol. Astropart. Phys. 05 (2006) 018.

[152] H. Peiris and R. Easther, J. Cosmol. Astropart. Phys. 07 (2006) 002

[153] W. H. Kinney, E. W. Kolb, A. Melchiorri, and A. Riotto, astro-ph/0605338.

[154] J. Martin and R. Ringeval, astro-ph/0605367.

[155] H. K. Erikssen et al., astro-ph/0606088.

[156] K. M. Huffenberger, H. K. Eriksen, and F. K. Hansen, astro-ph/0606538.

[157] A. R. Liddle, P. Mukherjee, and D. Parkinson, astro-ph/ 0608184.

[158] W. Hu, D. J. Eisenstein, and M. Tegmark, Phys. Rev. Lett. 80, 5255 (1998).

[159] A. Goobar, S. Hannestad, E. Mortsell, and H. Tu, J. Cosmol. Astropart. Phys. 06 (2006) 019.

[160] J. Lesgourges and S. Pastor, Phys. Rep. 429, 307 (2006).

[161] M. Fukugita, K. Ichikawa, M. Kawasaki, and O. Lahav, Phys. Rev. D 74, 027302 (2006).

[162] S. Hannestad and G. Raffelt, astro-ph/0607101.

[163] M. Cirelli and A. Strumia, astro-ph/0607086.

[164] S. King, hep-ph/0310204.

[165] Y. Ashie et al., Phys. Rev. D 71, 112005 (2005).

[166] W. A. Chiu, X. Fan, and J. P. Ostriker, Astrophys. J. 599, 759 (2003). 
[167] X. Fan et al., Astron. J. 132, 117 (2006).

[168] O. Zahn et al. (unpublished).

[169] M. Fukugita and P. J.E. Peebles, Astrophys. J. 616, 643 (2004).

[170] W. J. Percival et al., astro-ph/0608635 [Astrophys. J. Lett. (to be published)].

[171] M. Jarvis, B. Jain, G. Bernstein, and D. Dolney, Astrophys. J. 644, 71 (2006).

[172] A. F. Heavens, T. B. Kitching, and A. N. Taylor, astro-ph/ 0606568.

[173] N. Padmanabhan et al. (unpublished).

[174] U. Seljak et al., Phys. Rev. D 71, 043515 (2005).

[175] L. Verde et al., Mon. Not. R. Astron. Soc. 335, 432 (2002).

[176] J. P. Ostriker and A. Vale, astro-ph/0511816.
[177] W. A. Chiu, X. Fan, and J. P. Ostriker, Astrophys. J. 599, 759 (2003).

[178] M. Tegmark, A. J.S. Hamilton, M. A. Strauss, M. S. Vogeley, and A.S. Szalay, Astrophys. J. 499, 555 (1998).

[179] I. Szapudi and A. S. Szalay, Astrophys. J. Lett. 494, L41 (1998).

[180] I. Szapudi, S. Prunet, D. Pogosyan, A. S. Szalay, and J. R. Bond, Astrophys. J. 548, L115 (2001).

[181] M. Tegmark et al., Astrophys. J. 571, 191 (2002).

[182] S. Cole, K. B. Fisher, and D. H. Weinberg, Mon. Not. R. Astron. Soc. 267, 785 (1994).

[183] A. J.S. Hamilton, in The Evolving Universe, edited by D. Hamilton (Kluwer, Dordrecht, 1998), p. 185.

[184] B. M. S. Hansen et al., Astrophys. J. 574, L155 (2002). 